\title{
Prime exceptional divisors on holomorphic symplectic varieties and monodromy reflections
}

\author{
Eyal Markman
}

To the memory of Professor Masaki Maruyama

\begin{abstract}
Let $X$ be a projective irreducible holomorphic symplectic manifold. The second integral cohomology of $X$ is a lattice with respect to the Beauville-Bogomolov pairing. A divisor $E$ on $X$ is called a prime exceptional divisor if $E$ is reduced and irreducible and of negative Beauville-Bogomolov degree.

Let $E$ be a prime exceptional divisor on $X$. We first observe that associated to $E$ is a monodromy involution of the integral cohomology $H^{*}(X, \mathbb{Z})$, which acts on the second cohomology lattice as the reflection by the cohomology class $[E]$ of $E$.

We then specialize to the case where $X$ is deformation equivalent to the Hilbert scheme of length $n$ zero-dimensional subschemes of a $K 3$ surface, $n \geq 2$. We determine the set of classes of exceptional divisors on $X$. This leads to a determination of the closure of the movable cone of $X$.
\end{abstract}

\section{Contents}

1. Introduction . . . . . . . . . . . . . . . . . . . . . 345

2. Easy examples of monodromy-reflective line bundles . . . . . . . . . . . . 353

3. The monodromy reflection of a prime exceptional divisor . . . . . . . . 355

4. Holomorphic symplectic manifolds of $K 3^{[n]}$-type . . . . . . . . . . 358

5. Deformation equivalence . . . . . . . . . . . . . . 360

6. Monodromy invariants from Mukai's isomorphism . . . . . . . . . . . 370

7. Monodromy invariants of monodromy-reflective classes . . . . . . . . . . . 374

8. Numerical characterization of exceptional classes via Torelli . . . . . . . . 378

9. Conditions for the existence of slope-stable vector bundles . . . . . . . . 380

10. Examples of prime exceptional divisors . . . . . . . . . . . . . . . . . 387

11. Examples of noneffective monodromy-reflective classes . . . . . . . . . . . 397

References ............................. 400

\section{Introduction}

An irreducible holomorphic symplectic manifold is a simply connected compact Kähler manifold $X$, such that $H^{0}\left(X, \Omega_{X}^{2}\right)$ is generated by an everywhere nondegenerate holomorphic two-form (see [Be1], [Hu1]). The dimension of $X$ is even, say, $2 n$. The second integral cohomology of $X$ is a lattice with respect to the

Kyoto Journal of Mathematics, Vol. 53, No. 2 (2013), 345-403

DOI 10.1215/21562261-2081243, (C) 2013 by Kyoto University

Received April 2, 2012. Revised November 5, 2012. Accepted November 5, 2012.

2010 Mathematics Subject Classification: Primary 14D05, 14J60; Secondary 14J28, 53C26, 14C20. 
Beauville-Bogomolov pairing (see [Be1]). A divisor $E$ on $X$ is called a prime exceptional divisor if $E$ is reduced and irreducible and of negative BeauvilleBogomolov degree (see [Bou]).

\subsection{A prime exceptional divisor is monodromy reflective}

When $\operatorname{dim}(X)=2$, then $X$ is a Kähler $K 3$ surface. Let $E$ be a prime divisor of negative degree on $X$. Then $E$ is necessarily a smooth rational curve. Its degree is thus -2 . $E$ may be contracted, resulting in a surface $Y$ with an ordinary double point (see [BHPV, Chapter III]). A class $x \in H^{2}(X, \mathbb{Z})$ is primitive if it is not a multiple of another integral class by an integer larger than 1 . Let $c \in H^{2}(X, \mathbb{Z})$ be a primitive class of negative degree. Then $c$ has degree -2 if and only if the reflection $R_{c}: H^{2}(X, \mathbb{Q}) \rightarrow H^{2}(X, \mathbb{Q})$, given by

$$
R_{c}(x)=x-\frac{2(x, c)}{(c, c)} c,
$$

has integral values, since the lattice $H^{2}(X, \mathbb{Z})$ is even and unimodular.

Druel recently established the birational contractibility of a prime exceptional divisor $E$ on a projective irreducible holomorphic symplectic manifold $X$ of arbitrary dimension $2 n$. There exists a sequence of flops of $X$, resulting in a projective irreducible holomorphic symplectic manifold $X^{\prime}$, and a projective birational morphism $\pi: X^{\prime} \rightarrow Y$ onto a normal projective variety $Y$, such that the exceptional divisor $E^{\prime} \subset X^{\prime}$ of $\pi$ is the strict transform of $E$ (see [D] and Proposition 3.1 below). The result relies on the work of several authors, in particular, on Boucksom's [Bou] work on the divisorial Zariski decomposition and on recent results in the minimal model program (see [BCHM]).

Let $E$ be a prime exceptional divisor on a projective irreducible holomorphic symplectic manifold $X$. Let $c$ be the class of $E$ in $H^{2}(X, \mathbb{Z})$, and consider the reflection $R_{c}: H^{2}(X, \mathbb{Q}) \rightarrow H^{2}(X, \mathbb{Q})$, given by (1.1). Building on Druel's [D] result, we prove the following statement.

\section{THEOREM 1.1}

The reflection $R_{c}$ is a monodromy operator. In particular, $R_{c}$ is an integral isometry. Furthermore, $c$ is either a primitive class or two times a primitive class.

See Corollary 3.6 for a more detailed statement and a proof. The reflection $R_{c}$ arises as a monodromy operator as follows. Let $\operatorname{Def}(Y)$ be the Kuranishi deformation space of $Y$, and let $\bar{\psi}: \mathcal{Y} \rightarrow \operatorname{Def}(Y)$ be the semiuniversal family. Then $\operatorname{Def}(Y)$ is smooth, as is the fiber $Y_{t}$ of $\bar{\psi}$, over a generic point $t \in \operatorname{Def}(Y)$, and the smooth fiber $Y_{t}$ is deformation equivalent to $X$ (see [Na1]). Let $U \subset \operatorname{Def}(Y)$ be the complement of the discriminant locus. $R_{c}$ is exhibited as a monodromy operator of the local system $R_{\bar{\psi}_{*}}^{2} \mathbb{Z}$ over $U$. 


\subsection{Prime exceptional divisors in the $K 3^{[n]}$-type case}

Theorem 1.1 imposes a rather strong numerical condition on a class $c$ to be the class of a prime exceptional divisor. We get, for example, the following theorem. Assume that $X$ is deformation equivalent to the Hilbert scheme $S^{[n]}$ of length $n$ subschemes of a $K 3$ surface $S$. We will abbreviate this statement by saying that $X$ is of $K 3^{[n]}$-type. Assume that $n \geq 2$ and $X$ is projective.

\section{THEOREM 1.2}

Let e be a primitive class in $H^{2}(X, \mathbb{Z})$, with negative Beauville-Bogomolov degree $(e, e)<0$, such that some integer multiple of $e$ is the class of an irreducible divisor $E$. Then $(e, e)=-2$ or $(e, e)=2-2 n$. If $(e, e)=2-2 n$, then the class $(e, \bullet)$ in $H^{2}(X, \mathbb{Z})^{*}$ is divisible by $n-1$.

The theorem is related to Theorem 22 in the beautiful paper [HT2]. In particular, the case of fourfolds is settled in that paper. The hypothesis that the divisor $E$ is irreducible is necessary. There exist examples of pairs $(X, e)$, with $X$ of $K 3^{[n]}$ type $e \in H^{2}(X, \mathbb{Z})$, such that $2 e$ is effective, the reflection by $e$ is an integral reflection of $H^{2}(X, \mathbb{Z})$, but $2-2 n<(e, e)<-2$ (see [Ma4, Example 4.8]).

\section{DEFINITION 1.3}

An isometry $g$ of $H^{2}(X, \mathbb{Z})$ is called a monodromy operator if there exists a family $\mathcal{X} \rightarrow T$ (which may depend on $g$ ) of irreducible holomorphic symplectic manifolds, having $X$ as a fiber over a point $t_{0} \in T$, and such that $g$ belongs to the image of $\pi_{1}\left(T, t_{0}\right)$ under the monodromy representation. The monodromy group $\operatorname{Mon}^{2}(X)$ of $X$ is the subgroup of $O\left[H^{2}(X, \mathbb{Z})\right]$ generated by all the monodromy operators.

\section{DEFINITION 1.4}

(1) A class $e \in H^{2}(X, \mathbb{Z})$ is said to be monodromy reflective if $e$ is primitive and the reflection $R_{e}(x):=x-\frac{2(x, e)}{(e, e)} e$, with respect to the class $e$, belongs to $\operatorname{Mon}^{2}(X)$.

(2) A line bundle $L$ is said to be monodromy reflective, if the class $c_{1}(L)$ is.

Theorem 1.2 is an immediate consequence of Theorem 1.1 and the following characterization of monodromy-reflective line bundles on $X$ of $K 3^{[n]}$-type.

\section{PROPOSITION 1.5}

Let $e \in H^{2}(X, \mathbb{Z})$ be a primitive class of negative degree $(e, e)$. Then the reflection $R_{e}$ belongs to $\operatorname{Mon}^{2}(X)$ if and only if e has one of the following two properties:

(1) $(e, e)=-2$, or

(2) $(e, e)=2-2 n$, and $n-1$ divides the class $(e, \bullet) \in H^{2}(X, \mathbb{Z})^{*}$. 
The proposition is proven in Section 4 . A class $e \in H^{1,1}(X, \mathbb{Z})$ is said to be $\mathbb{Q}$ effective, if some nonzero integer multiple of $e$ is the class of an effective divisor. Examples of monodromy-reflective line bundles, which are not $\mathbb{Q}$-effective, are exhibited in Section 11.

\subsection{A classification of monodromy-reflective line bundles}

When $X$ is a $K 3$ surface, a monodromy-reflective line bundle $L$ has degree -2 , and precisely one of $L$ or $L^{-1}$ is isomorphic to $\mathcal{O}_{X}(E)$, where $E$ is an effective divisor (see [BHPV, Chapter VIII, Proposition 3.6]). If $\operatorname{Pic}(X)$ is cyclic, then $E$ is necessarily a smooth rational curve. We may deform to this case upon deforming the pair $(X, L)$ to a nearby deformation equivalent pair.

Monodromy-reflective line bundles of degree $2-2 n$, over $X$ of $K 3^{[n]}$-type, need not be $\mathbb{Q}$-effective if $n>1$. Whether the line bundle is or is not $\mathbb{Q}$-effective depends on a monodromy invariant defined in Proposition 1.7 below. The definition depends on the following theorem.

The topological $K$-group $K(S)$ of a $K 3$ surface $S$, endowed with the Mukai pairing $(v, w):=-\chi\left(v^{\vee} \otimes w\right)$, is called the Mukai lattice. $K(S)$ is a rank 24 even unimodular lattice isometric to the orthogonal direct sum $\widetilde{\Lambda}:=E_{8}(-1)^{\oplus 2} \oplus U^{\oplus 4}$, where $E_{8}(-1)$ is the negative definite $E_{8}$ lattice and $U$ is the rank 2 lattice with Gram matrix $\left(\begin{array}{ll}0 & 1 \\ 1 & 0\end{array}\right)$.

Let $\Lambda:=E_{8}(-1)^{\oplus 2} \oplus U^{\oplus 3} \oplus \mathbb{Z} \delta$, with $(\delta, \delta)=2-2 n$. Then $H^{2}(X, \mathbb{Z})$ is isometric to $\Lambda$, for any $X$ of $K 3^{[n]}$-type, $n>1$ (see [Be1]). Let $O(\Lambda, \widetilde{\Lambda})$ be the set of primitive isometric embeddings $\iota: \Lambda \hookrightarrow \widetilde{\Lambda}$. $O(\widetilde{\Lambda})$ acts on $O(\Lambda, \widetilde{\Lambda})$ by compositions. If $n-1$ is a prime power, then $O(\Lambda, \widetilde{\Lambda})$ consists of a single $O(\widetilde{\Lambda})$-orbit. The Euler number $\eta:=\eta(n-1)$ is the number of distinct primes $p_{1}, \ldots, p_{\eta}$ in the prime factorization $n-1=p_{1}^{e_{1}} \cdots p_{\eta}^{e_{\eta}}$, with positive integers $e_{i}$. For $n>2$, there are $2^{\eta-1}$ distinct $O(\widetilde{\Lambda})$-orbits in $O(\Lambda, \widetilde{\Lambda})$ (see [O] or [Ma4, Lemma 4.3]).

\section{THEOREM 1.6 ([Ma4, THEOREM 1.10])}

An irreducible holomorphic symplectic manifold $X$ of $K 3^{[n]}$-type, $n \geq 2$, comes with a natural choice of an $O(\widetilde{\Lambda})$-orbit of primitive isometric embeddings of $H^{2}(X, \mathbb{Z})$ in $\widetilde{\Lambda}$. This orbit is monodromy invariant; that is, $\iota: H^{2}(X, \mathbb{Z}) \hookrightarrow \widetilde{\Lambda}$ belongs to this orbit if and only if $\iota \circ g$ does, for all $g \in \operatorname{Mon}^{2}(X)$.

Let $S$ be a $K 3$ surface, let $H$ be an ample line bundle on $S$, and let $v \in K(S)$ be a primitive class satisfying $(v, v)=2 n-2, n \geq 2$. Assume that $X:=M_{H}(v)$ is a smooth and compact moduli space of $H$-stable sheaves of class $v$. Then $X$ is of $K 3^{[n]}$-type and the orbit in the theorem is that of Mukai's isometry $\iota: H^{2}\left(M_{H}(v), \mathbb{Z}\right) \rightarrow v^{\perp}$, where $v^{\perp} \subset K(S)$ is the sublattice orthogonal to $v$ (see [Ma4, Theorem 1.14] or Theorem 2.1 below). The monodromy invariance of the $O(K(S))$-orbit of Mukai's isometry uniquely characterizes the orbit in the above theorem, for every $X$ of $K 3^{[n]}$-type.

Let $X$ be of $K 3^{[n]}$-type, $n>1$. Let $I_{n}^{\prime \prime}(X) \subset H^{2}(X, \mathbb{Z})$ be the subset of monodromy-reflective classes of degree $2-2 n$, and let $e$ be a class in $I_{n}^{\prime \prime}(X)$. 
Choose a primitive isometric embedding $\iota: H^{2}(X, \mathbb{Z}) \hookrightarrow \widetilde{\Lambda}$, in the natural orbit of Theorem 1.6. Choose a generator $v$ of the rank 1 sublattice of $\widetilde{\Lambda}$ orthogonal to the image of $\iota$. Then $(v, v)=2 n-2$. Indeed, $(v, v)>0$, since the signature of $\widetilde{\Lambda}$ is $(4,20)$, and $(v, v)$ is equal to the order of $(\mathbb{Z} v)^{*} / \mathbb{Z} v$, which is equal to the order of $H^{2}(X, \mathbb{Z})^{*} / H^{2}(X, \mathbb{Z})$, which is $2 n-2$. Let $\rho$ be the positive integer, such that $(e+v) / \rho$ is an integral and primitive class in $\widetilde{\Lambda}$. Define the integer $\sigma$ similarly using $e-v$. Let $\operatorname{div}(e, \bullet)$ be the integer in $\{(e, e) / 2,(e, e)\}$, such that the class $(e, \bullet) / \operatorname{div}(e, \bullet)$ is an integral and primitive class in $H^{2}(X, \mathbb{Z})^{*}$. Given a rational number $m$, let $\mathcal{F}(m)$ be the set of unordered pairs $\{r, s\}$ of positive integers, such that $r s=m$ and $\operatorname{gcd}(r, s)=1$. If $m$ is not a positive integer, then $\mathcal{F}(m)$ is empty. Set

$$
\Sigma_{n}^{\prime \prime}:=\mathcal{F}(n-1) \cup \mathcal{F}([n-1] / 2) \cup \mathcal{F}([n-1] / 4) .
$$

Note that $\Sigma_{n}^{\prime \prime}$ is a singleton if and only if $n=2$ or $n-1$ is an odd prime power.

\section{PROPOSITION 1.7}

If $\operatorname{div}(e, \bullet)=n-1$ and $n$ is even, set $\{r, s\}:=\{\rho, \sigma\}$. Otherwise, set $\{r, s\}:=$ $\{\rho / 2, \sigma / 2\}$. Then $\{r, s\}$ is a pair of relatively prime integers in $\Sigma_{n}^{\prime \prime}$, and the function

$$
r s: I_{n}^{\prime \prime}(X) \longrightarrow \Sigma_{n}^{\prime \prime},
$$

sending the class $e$ to the unordered pair $\{r, s\}$, is monodromy invariant. The function rs is surjective, if $n \equiv 1$ modulo 8 , and its image is $\mathcal{F}(n-1) \cup \mathcal{F}([n-$ 1]/2) otherwise.

The proposition is proven in Lemmas 7.1 and 7.3. A more conceptual definition of the monodromy-invariant $r s$ is provided in the statements of these lemmas. The proof relies on the classification of the isometry classes of all possible pairs $(\widetilde{L}, e)$, where $\widetilde{L}$ is the saturation in $\widetilde{\Lambda}$ of the $\operatorname{rank} 2$ sublattice $\operatorname{span}\{e, v\}$. The classification is summarized in Table 1 . We finally arrive at the classification of monodromy-reflective line bundles.

\section{PROPOSITION 1.8}

Let $X$ be of $K 3^{[n]}$-type, and let $L$ be a monodromy-reflective line bundle. Set $e:=c_{1}(L)$.

(1) If $(e, e)=2-2 n$, then the $\operatorname{Mon}^{2}(X)$-orbit of the class e is determined by $\operatorname{div}(e, \bullet)$ and the value $\operatorname{rs}(e)$.

(2) If $(e, e)=-2$, then the $\operatorname{Mon}^{2}(X)$-orbit of the class $e$ is determined by $\operatorname{div}(e, \bullet)$.

Part (1) is proven in Lemmas 7.1 and 7.3. Part (2) is [Ma3, Lemma 8.9]. 


\subsection{A numerical characterization of exceptional classes}

Let $\left(X_{1}, L_{1}\right)$ and $\left(X_{2}, L_{2}\right)$ be two pairs as in Proposition 1.8. Set $e_{i}:=c_{1}\left(L_{i}\right)$. If $\left(e_{i}, e_{i}\right)=2-2 n, \operatorname{div}\left(e_{1}, \bullet\right)=\operatorname{div}\left(e_{2}, \bullet\right)$, and $r s\left(e_{1}\right)=r s\left(e_{2}\right)$, then the pairs $\left(X_{1}, L_{1}\right)$ and $\left(X_{2}, L_{2}^{\epsilon}\right)$ are deformation equivalent (requiring $e_{i}$ to preserve its Hodge type along the deformation), for $\epsilon=1$ or $\epsilon=-1$, by Proposition 1.8 and Lemma 5.17. (The proof of the latter depends on the Torelli theorem; see [Ver]). Furthermore, if $X_{1}$ is projective and $L_{1}^{\otimes k} \cong \mathcal{O}_{X_{1}}\left(E_{1}\right)$, for some $k>0$ and a prime exceptional divisor $E_{1}$, then a generic small deformation $(X, L)$ of $\left(X_{2}, L_{2}\right)$ consists of $L$ satisfying $L^{\otimes d} \cong \mathcal{O}_{X}(E)$ for a prime exceptional divisor $E$ and for $d=k$ or $d=-k$ (see Proposition 5.14). This leads us to the numerical characterization of exceptional line bundles described in this section.

Let $X$ be an irreducible holomorphic symplectic manifold of $K 3^{[n]}$-type, $n \geq 2$. Let $L$ be a monodromy-reflective line bundle on $X, e:=c_{1}(L)$, and let $R_{e}$ be the reflection by $e . R_{e}$ preserves the Hodge structure and so acts on $H^{1,1}(X) \cong$ $H^{1}\left(X, T_{X}\right)$. The Kuranishi deformation space $\operatorname{Def}(X)$ is an open neighborhood of zero in $H^{1}\left(X, T_{X}\right)$, which may be chosen to be $R_{e}$-invariant. Hence, $R_{e}$ acts on $\operatorname{Def}(X)$. The local Kuranishi deformation space $\operatorname{Def}(X, L)$, of the pair $(X, L)$, is the smooth divisor $D_{e} \subset \operatorname{Def}(X)$ of fixed points of $R_{e}$.

\section{DEFINITION 1.9}

Let $h \in H^{2}(X, \mathbb{R})$ be a Kähler class. A line bundle $L \in \operatorname{Pic}(X)$ is called numerically exceptional if its first Chern class $e:=c_{1}(L)$ is a primitive class in $H^{2}(X, \mathbb{Z})$, satisfying $(h, e)>0$ and the following properties. The Beauville-Bogomolov degree is either $(e, e)=-2$, or $(e, e)=2-2 n$ and $n:=\operatorname{dim}_{\mathbb{C}}(X) / 2>2$. In the latter case one of the following properties holds:

(1) $\operatorname{div}(e, \bullet)=2 n-2$ and $r s(e)=\{1, n-1\}$;

(2) $\operatorname{div}(e, \bullet)=2 n-2$ and $r s(e)=\{2,(n-1) / 2\}$; we must have $n \equiv 3$ (modulo 4) for the pair $r s(e)$ to be relatively prime;

(3) $\operatorname{div}(e, \bullet)=n-1, n$ is even, and $r s(e)=\{1, n-1\}$;

(4) $\operatorname{div}(e, \bullet)=n-1, n$ is odd, and $r s(e)=\{1,(n-1) / 2\}$.

A cohomology class $e \in H^{1,1}(X, \mathbb{Z})$ is numerically exceptional, if $e=c_{1}(L)$, for a numerically exceptional line bundle $L$.

\section{DEFINITION 1.10}

(1) A line bundle $L \in \operatorname{Pic}(X)$ is called stably prime exceptional, if there exists a closed complex analytic subset $Z \subset D_{e}$ of codimension $\geq 1$, such that the linear system $\left|L_{t}\right|$ consists of a prime-exceptional divisor $E_{t}$, for all $t \in\left[D_{e} \backslash Z\right]$.

(2) $L$ is said to be stably $\mathbb{Q}$-effective, if there exists a nonzero integer $k$, such that the linear system $\left|L_{t}^{k}\right|$ is nonempty, for all $t \in D_{e}$.

If $E$ is a prime exceptional divisor on a projective irreducible holomorphic symplectic manifold $X$, then $\mathcal{O}_{X}(E)$ is stably prime exceptional, by Proposition 5.2. 
Let $L$ be a line bundle on an irreducible holomorphic symplectic manifold $X$ of $K 3^{[n]}$-type with a primitive first Chern class. Recall that a necessary condition for the linear system $\left|L^{k}\right|$ to consist of an exceptional divisor $E$ is that $L$ is monodromy reflective (see Definition 1.4), by Theorem 1.1. Assume that $L$ is monodromy reflective. Set $e:=c_{1}(L)$. Let $D_{e} \subset \operatorname{Def}(X)$ be the divisor fixed by the reflection $R_{e}$.

THEOREM 1.11

(1) Assume that $L$ is numerically exceptional. Then $L^{k}$ is stably prime exceptional, where $k$ is determined as follows. If the degree of $L$ is $2-2 n$, then

$$
k= \begin{cases}2 & \text { if } \operatorname{div}(e, \bullet)=2 n-2 \text { and } r s(e)=\{1, n-1\}, \\ 1 \quad \text { if } \operatorname{div}(e, \bullet)=2 n-2 \text { and } r s(e)=\{2,(n-1) / 2\}, \\ 1 \quad \text { if } \operatorname{div}(e, \bullet)=n-1 .\end{cases}
$$

If the degree of $L$ is -2 , then

$$
k= \begin{cases}2 & \text { if } \operatorname{div}(e, \bullet)=2 \text { and } n=2 \\ 1 & \text { if } \operatorname{div}(e, \bullet)=2 \text { and } n>2 \\ 1 & \text { if } \operatorname{div}(e, \bullet)=1\end{cases}
$$

(2) If $L$ is not numerically exceptional, then $L$ is not stably $\mathbb{Q}$-effective. That is, for every nonzero integer $k$, there exists a dense open subset $U^{k}$ of $D_{e}$, such that $H^{0}\left(X_{t}, L_{t}^{k}\right)$ vanishes, for all $t \in U^{k}$.

See Remark 4.2 for the Euler characteristic $\chi\left(L^{k}\right)$. Note that in Theorem 1.11(1) $L^{k}$ is effective as well, for the specified integer $k$, by the semicontinuity theorem.

Theorem 1.11 is proven in Section 8. The proof relies on both the Torelli theorem (see [Ver]) and the examples worked out in Sections 10 and 11. We exhibit an example of a pair $(X, L)$, for each possible value of the monodromy invariants $(e, e), \operatorname{div}(e, \bullet)$, and $r s(e)$, and verify Theorem 1.11 for $(X, L)$. All values of the monodromy invariants are realized by examples where $X$ is a smooth and projective moduli space of sheaves on a $K 3$ surface. See Table 2 for a reference to an example for each value of the monodromy invariants.

The vanishing in Theorem 1.11(2) is verified in the examples as follows. In all the examples of monodromy-reflective but nonnumerically exceptional line bundles considered in Section 11, $X$ admits a birational involution $\iota: X \rightarrow X$, inducing the reflection $R_{e}$.

The following simple observation is proven in Section 11.

\section{OBSERVATION 1.12}

If $L$ is a monodromy-reflective line bundle on $X$, and there exists a bimeromorphic involution $\iota: X \rightarrow X$ inducing the reflection $R_{e}, e=c_{1}(L)$, then the line bundle $L$ is not $\mathbb{Q}$-effective. 


\subsection{Cones}

Let $X$ be a projective irreducible holomorphic symplectic manifold. Set $N^{1}(X):=$ $H^{1,1}(X, \mathbb{Z}) \otimes_{\mathbb{Z}} \mathbb{R}$, and let $\mathcal{C}_{X}^{1,1}$ be the connected component of the cone $\{\lambda \in$ $\left.N^{1}(X):(\lambda, \lambda)>0\right\}$, which contains the ample cone. Denote by $\overline{\mathcal{C}}_{X}^{1,1}$ its closure. A divisor $D$ on $X$ is called movable if the base locus of the linear system $|D|$ has codimension $\geq 2$ in $X$. Denote by $\mathcal{M V _ { X }}$ the convex cone in $N^{1}(X)$ generated by classes of movable divisors. Let $\overline{\mathcal{M V}}_{X}$ be its closure in $N^{1}(X)$. Then $\overline{\mathcal{M V}}_{X}$ is equal to the subcone of $\overline{\mathcal{C}}_{X}^{1,1}$, consisting of classes $\lambda$, such that $(\lambda,[E]) \geq 0$ for every prime exceptional divisor $E$ (see [Bou], [Ma6, Lemma 6.22]).

The closure of the movable cone can be described also in terms of the set of stably prime exceptional divisors. $\overline{\mathcal{M V}}_{X}$ is the subcone of $\overline{\mathcal{C}}_{X}^{1,1}$, consisting of classes $\lambda$ such that $(\lambda, e) \geq 0$, for every stably prime exceptional class $e[\mathrm{Ma} 6$, Theorem 6.17, Lemma 6.22]. Hence, Theorem 1.11 above determines the closure of the movable cone. Furthermore, a stably prime exceptional class $e$ is prime exceptional if and only if the hyperplane orthogonal to $e$ intersects $\overline{\mathcal{M V}}_{X}$ along a face of codimension one of the latter (see [Ma6, Lemma 6.20]). In this sense Theorem 1.11 determines the set of classes of prime exceptional divisors.

\subsection{The structure of the paper}

The paper is organized as follows. In Section 2 we provide a sequence of easy examples of monodromy reflective line bundles on moduli spaces of sheaves on $K 3$ surfaces. We calculate their invariants, and determine whether or not they are effective, illustrating Theorem 1.11.

In Section 3 we prove Theorem 1.1 stating that associated to a prime exceptional divisor $E$ is a monodromy involution of the integral cohomology $H^{*}(X, \mathbb{Z})$, which acts on the second cohomology lattice as the reflection by the cohomology class $[E]$ of $E$ (see Corollary 3.6). In Section 4 we specialize to the $K 3^{[n]}$-type case, $n \geq 2$, and prove Theorem 1.2 about the possible degrees of prime exceptional divisors.

Let $\left(X_{i}, E_{i}\right), i=1,2$, be two pairs, each consisting of an irreducible holomorphic symplectic manifold $X_{i}$ and a prime exceptional divisor $E_{i}$. Let $e_{i} \in$ $H^{2}(X, \mathbb{Z})$ be the class of $E_{i}$. In Section 5 we define two notions of deformation equivalence:

(1) deformation equivalence of the two pairs $\left(X_{i}, E_{i}\right), i=1,2$;

(2) deformation equivalence of the two pairs $\left(X_{i}, e_{i}\right), i=1,2$.

We relate these two notions via Torelli.

In Section 6 we return to the case where $X$ is of $K 3^{[n]}$-type, $n \geq 2$. We associate, to each monodromy-reflective class $e \in H^{2}(X, \mathbb{Z})$ of degree $2-2 n$, an isometry class of a pair $(\widetilde{L}, \tilde{e})$, consisting of a rank 2 integral lattice $\widetilde{L}$ of signature $(1,1)$ and a primitive class $\tilde{e} \in \widetilde{L}$, with $(\tilde{e}, \tilde{e})=2-2 n$. The isometry class of the pair $(\widetilde{L}, \tilde{e})$ is a monodromy invariant, denoted by $f(X, e)$. In Section 7 we calculate the monodromy invariant $f(X, e)$ explicitly as the function $r s$ in Proposition 1.8. We then prove Proposition 1.8. 
In Section 8 we prove Theorem 1.11, which provides a numerical characterization of exceptional classes.

Sections 9, 10, and 11 are devoted to examples of monodromy-reflective line bundles over moduli spaces of stable sheaves. In Section 9 we study the exceptional locus of Jun Li's [Li] morphism from certain moduli spaces, of GiesekerMaruyama $H$-stable sheaves on a $K 3$-surface, to the Uhlenbeck-Yau compactifications of the moduli spaces of $H$-slope-stable vector bundles.

In Section 10 we exhibit an example of a prime exceptional divisor for each value of the invariants of a monodromy-reflective line bundle $L$, for which $L$ is stated to be stably $\mathbb{Q}$-effective in Theorem 1.11 .

In Section 11 we exhibit an example of a monodromy-reflective line bundle $L$, which is not $\mathbb{Q}$-effective, for each value of the invariants for which $L$ is stated not to be $\mathbb{Q}$-effective in Theorem 1.11.

\section{Easy examples of monodromy-reflective line bundles}

In Section 2.1 we review basic facts about moduli spaces of coherent sheaves on $K 3$ surfaces. In Section 2.2 we briefly describe a sequence of examples of pairs $(X, e)$, with $X$ of $K 3^{[n]}$-type, where $e$ is a monodromy-reflective class of degree $2-2 n$ with $\operatorname{div}(e, \bullet)=2 n-2$, for each $n \geq 2$ and for each value of the invariant $r s$. For details and references, as well as for examples of degree -2 , or with $\operatorname{div}(e, \bullet)=n-1$, see Sections 10 and 11.

\subsection{The Mukai isomorphism}

The group $K(S)$, endowed with the Mukai pairing

$$
(v, w):=-\chi\left(v^{\vee} \otimes w\right),
$$

is called the Mukai lattice. Let us recall Mukai's notation for elements of $K(S)$. Identify the group $K(S)$ with $H^{*}(S, \mathbb{Z})$, via the isomorphism sending a class $F$ to its Mukai vector $\operatorname{ch}(F) \sqrt{t d_{S}}$. Using the grading of $H^{*}(S, \mathbb{Z})$, the Mukai vector is

$$
\left(\operatorname{rank}(F), c_{1}(F), \chi(F)-\operatorname{rank}(F)\right),
$$

where the rank is considered in $H^{0}$ and $\chi(F)-\operatorname{rank}(F)$ in $H^{4}$ via multiplication by the orientation class of $S$. The homomorphism $\operatorname{ch}(\bullet) \sqrt{t d_{S}}: K(S) \rightarrow H^{*}(S, \mathbb{Z})$ is an isometry with respect to the Mukai pairing on $K(S)$ and the pairing

$$
\left(\left(r^{\prime}, c^{\prime}, s^{\prime}\right),\left(r^{\prime \prime}, c^{\prime \prime}, s^{\prime \prime}\right)\right)=\int_{S} c^{\prime} \cup c^{\prime \prime}-r^{\prime} \cup s^{\prime \prime}-s^{\prime} \cup r^{\prime \prime}
$$

on $H^{*}(S, \mathbb{Z})$ (by the Hirzebruch-Riemann-Roch theorem). For example, (1, 0, 1 $n)$ is the Mukai vector in $H^{*}(S, \mathbb{Z})$, of the ideal sheaf of a length $n$ subscheme. Mukai defines a weight 2 Hodge structure on the Mukai lattice $H^{*}(S, \mathbb{Z})$, and hence on $K(S)$, by extending that of $H^{2}(S, \mathbb{Z})$ so that the direct summands $H^{0}(S, \mathbb{Z})$ and $H^{4}(S, \mathbb{Z})$ are of type $(1,1)$.

Let $v \in K(S)$ be a primitive class with $c_{1}(v)$ of Hodge-type $(1,1)$. There is a system of hyperplanes in the ample cone of $S$, called $v$-walls, that is countable 
but locally finite (see [HL, Chapter 4.C]). An ample class is called v-generic if it does not belong to any $v$-wall. Choose a $v$-generic ample class $H$. Let $M_{H}(v)$ be the moduli space of $H$-stable sheaves on the $K 3$ surface $S$ with class $v$. When nonempty, the moduli space $M_{H}(v)$ is a smooth projective irreducible holomorphic symplectic variety of $K 3^{[n]}$-type, with $n=((v, v)+2) / 2$. This result is due to several people, including Huybrechts, Mukai, O'Grady, and Yoshioka. It can be found in its final form in [Y2].

Over $S \times M_{H}(v)$ there exists a universal sheaf $\mathcal{F}$, possibly twisted with respect to a nontrivial Brauer class pulled back from $M_{H}(v)$. Associated to $\mathcal{F}$ is a class $[\mathcal{F}]$ in $K\left(S \times M_{H}(v)\right)$ (see [Ma2, Definition 26]). Let $\pi_{i}$ be the projection from $S \times M_{H}(v)$ onto the $i$ th factor. Assume that $(v, v)>0$. The second integral cohomology $H^{2}\left(M_{H}(v), \mathbb{Z}\right)$, its Hodge structure, and its Beauville-Bogomolov pairing are all described by Mukai's Hodge isometry

$$
\theta: v^{\perp} \longrightarrow H^{2}\left(M_{H}(v), \mathbb{Z}\right)
$$

given by $\theta(x):=c_{1}\left(\pi_{2 !}\left\{\pi_{1}^{!}\left(x^{\vee}\right) \otimes[\mathcal{F}]\right\}\right)$ (see $\left.[\mathrm{Y} 2]\right)$.

Let $\widetilde{\Lambda}$ be the unimodular lattice $E_{8}(-1)^{\oplus 2} \oplus U^{\oplus 4}$, where $U$ is the rank two unimodular hyperbolic lattice. $\widetilde{\Lambda}$ is isometric to the Mukai lattice of a $K 3$ surface. Let $X$ be an irreducible holomorphic symplectic manifold of $K 3^{[n]}$-type, $n \geq 2$. Recall that $X$ comes with a natural choice of an $O(\widetilde{\Lambda})$-orbit of primitive isometric embeddings of $H^{2}(X, \mathbb{Z})$ in $\widetilde{\Lambda}$, by Theorem 1.6.

\section{THEOREM 2.1 ([Ma4, THEOREM 1.14])}

When $X$ is isomorphic to the moduli space $M_{H}(v)$, of $H$-stable sheaves on a $K 3$ surface of class $v \in K(S)$, then the above-mentioned $O(\widetilde{\Lambda})$-orbit is that of the composition

$$
H^{2}\left(M_{H}(v), \mathbb{Z}\right) \stackrel{\theta^{-1}}{\longrightarrow} v^{\perp} \subset K(S) \cong \widetilde{\Lambda}
$$

where $\theta^{-1}$ is the inverse of the Mukai isometry given in (2.2).

The combination of Theorems 1.6 and 2.1 is an example of the following metaprinciple guiding our study of the monodromy of holomorphic symplectic varieties of $K 3^{[n]}$-type.

Any topological construction, which can be performed uniformly and naturally for all smooth and compact moduli spaces of sheaves on any $K 3$ surface $S$, and which is invariant under symmetries induced by equivalences of derived categories of K3-surfaces, is monodromy invariant.

\subsection{A representative sequence of examples}

Let $S$ be a projective $K 3$ surface with a cyclic Picard group generated by an ample line bundle $H$. Fix integers $r$ and $s$ satisfying $s \geq r \geq 1$ and $\operatorname{gcd}(r, s)=1$. Let $X$ be the moduli space $M_{H}(r, 0,-s)$. Then $X$ is a projective irreducible holomorphic symplectic manifold of $K 3^{[n]}$-type with $n=1+r s$ (see [Y2]). Set $e:=\theta(r, 0, s)$. The weight two integral Hodge structure $H^{2}\left(M_{H}(r, 0,-s), \mathbb{Z}\right)$ is 
Hodge-isometric to the orthogonal direct sum $H^{2}(S, \mathbb{Z}) \oplus \mathbb{Z} e$, and the class $e$ is monodromy reflective of Hodge-type $(1,1),(e, e)=2-2 n, \operatorname{div}(e, \bullet)=2 n-2$, and $r s(e)=\{r, s\}$.

When $r=1$, then $X=S^{[n]}$ is the Hilbert scheme. Let $E \subset S^{[n]}$ be the big diagonal. Then $E$ is a prime divisor, which is the exceptional locus of the HilbertChow morphism $\pi: S^{[n]} \rightarrow S^{(n)}$ onto the $n$th symmetric product of $S$. The equality $e=[E] / 2$ was proven in $[\mathrm{Be} 1]$.

When $r=2$, let $E \subset M_{H}(2,0,-s)$ be the locus of $H$-stable sheaves which are not locally free. Then $E$ is a prime divisor, which is the exceptional locus of Jun Li's morphism from $M_{H}(2,0,-s)$ onto the Uhlenbeck-Yau compactification of the moduli space of $H$-slope-stable vector bundles of that class. The equality $e=[E]$ holds, by Lemma 10.16 .

When $r \geq 3$, let Exc $\subset M_{H}(r, 0,-s)$ be the locus of $H$-stable sheaves which are not locally free or not $H$-slope-stable. Then Exc is a closed algebraic subset of $M_{H}(r, 0,-s)$ of codimension $\geq 2$, by Lemma 9.5. Jun Li's morphism is thus not a divisorial contraction. Set $U:=X \backslash$ Exc. Let $\iota: U \rightarrow U$ be the regular involution, which sends a locally free $H$-slope-stable sheaf $F$ to the dual sheaf $F^{*}$. The restriction homomorphism from $H^{2}(X, \mathbb{Z})$ to $H^{2}(U, \mathbb{Z})$ is an isomorphism, and the induced involution $\iota^{*}$ of $H^{2}(X, \mathbb{Z})$ is the reflection by the class $e$, by Proposition 11.1. The class $e$ is thus not $\mathbb{Q}$-effective, by Observation 1.12.

\section{The monodromy reflection of a prime exceptional divisor}

Let $X$ be a projective irreducible holomorphic symplectic manifold, and let $E$ be a reduced and irreducible divisor with negative Beauville-Bogomolov degree. The following result is due to S. Bouksom and S. Druel.

PROPOSITION 3.1 ([D, PROPOSITION 1.4])

There exists a sequence of flops of $X$, resulting in a smooth birational model $X^{\prime}$ of $X$, such that the strict transform $E^{\prime}$ of $E$ in $X^{\prime}$ is contractible via a projective birational morphism $\pi: X^{\prime} \rightarrow Y$ to a normal projective variety $Y$. The exceptional locus of $\pi$ is equal to the support of $E^{\prime}$.

The divisor $E$ is assumed to be exceptional, rather than to have negative BeauvilleBogomolov degree, in the statement of [D, Proposition 1.4]. The technical term is exceptional in the sense of [Bou, Definition 3.10] and is a precise measure of rigidity. Boucksom characterized exceptional divisors on irreducible holomorphic symplectic varieties by the following property, which we will use as a definition (see [Bou, Theorem 4.5]).

\section{DEFINITION 3.2}

A rational divisor $E \in \operatorname{Div}(X) \otimes_{\mathbb{Z}} \mathbb{Q}$ is exceptional, if $E=\sum_{i=1}^{k} n_{i} E_{i}$, with positive rational coefficients $n_{i}$, prime divisors $E_{i}$, and a negative definite Gram $\operatorname{matrix}\left(\left[E_{i}\right],\left[E_{j}\right]\right)$. 
In particular, a prime divisor is exceptional if and only if it has negative BeauvilleBogomolov degree. Hence, we may replace in the above proposition the hypothesis that $E$ is exceptional by the hypothesis that $E$ has negative Beauville-Bogomolov degree.

\section{DEFINITION 3.3}

A primitive class $e \in H^{2}(X, \mathbb{Z})$ is (prime) exceptional if some positive multiple of $e$ is the class of a (prime) exceptional divisor. A line bundle $L \in \operatorname{Pic}(X)$ is (prime) exceptional if $c_{1}(L)$ is.

Let $\operatorname{Def}\left(X^{\prime}\right)$ and $\operatorname{Def}(Y)$ be the Kuranishi deformation spaces of $X^{\prime}$ and $Y$. Denote by $\psi: \mathcal{X} \rightarrow \operatorname{Def}\left(X^{\prime}\right)$ the semiuniversal deformation of $X^{\prime}$, denote by $0 \in \operatorname{Def}\left(X^{\prime}\right)$ the point with fiber $X^{\prime}$, and let $X_{t}$ be the fiber over $t \in \operatorname{Def}\left(X^{\prime}\right)$. Let $\bar{\psi}: \mathcal{Y} \rightarrow \operatorname{Def}(Y)$ be the semiuniversal deformation of $Y$, let $\overline{0} \in \operatorname{Def}(Y)$ be its special point with fiber $Y$, and let $Y_{t}$ be the fiber over $t \in \operatorname{Def}(Y)$.

The variety $Y$ necessarily has rational Gorenstein singularities, by $[\mathrm{Be} 2$, Proposition 1.3]. The morphism $\pi: X^{\prime} \rightarrow Y$ deforms as a morphism $\nu$ of the semiuniversal families, which fits in a commutative diagram

$$
\begin{array}{ccc}
\mathcal{X} & \stackrel{\nu}{\rightarrow} & \mathcal{Y} \\
\psi \downarrow & & \bar{\psi} \downarrow \\
\operatorname{Def}\left(X^{\prime}\right) \stackrel{f}{\rightarrow} & \operatorname{Def}(Y)
\end{array}
$$

by [KM, Proposition 11.4]. The following is a fundamental theorem of Namikawa.

\section{THEOREM 3.4 ([Na1, THEOREM 2.2])}

The Kuranishi deformation spaces $\operatorname{Def}\left(X^{\prime}\right)$ and $\operatorname{Def}(Y)$ are both smooth of the same dimension. They can be replaced by open neighborhoods of $0 \in \operatorname{Def}\left(X^{\prime}\right)$ and $\overline{0} \in \operatorname{Def}(Y)$, and denoted again by $\operatorname{Def}\left(X^{\prime}\right)$ and $\operatorname{Def}(Y)$, in such a way that there exists a natural proper surjective map $f: \operatorname{Def}\left(X^{\prime}\right) \rightarrow \operatorname{Def}(Y)$ with finite fibers. Moreover, for a generic point $t \in \operatorname{Def}\left(X^{\prime}\right), Y_{f(t)}$ is isomorphic to $X_{t}$.

The morphism $f: \operatorname{Def}\left(X^{\prime}\right) \rightarrow \operatorname{Def}(Y)$ is in fact a branched Galois covering, by [Ma5, Lemma 1.2]. The Galois group $G$ is a product of Weyl groups of finite type, by [Ma5, Theorem 1.4] (see also [Na2]). Furthermore, $G$ acts on $H^{*}\left(X^{\prime}, \mathbb{Z}\right)$ via monodromy operators preserving the Hodge structure. When the exceptional locus of $\pi: X^{\prime} \rightarrow Y$ contains a single irreducible component of codimension one in $X^{\prime}$, then the Galois group $G$ is $\mathbb{Z} / 2 \mathbb{Z}$.

Let $\Sigma \subset Y$ be the singular locus. The dissident locus $\Sigma_{0} \subset \Sigma$ is the locus along which the singularities of $Y$ fail to be of $A D E$ type. $\Sigma_{0}$ is a closed subvariety of $\Sigma$.

PROPOSITION 3.5 ([Na1, PROPOSITION 1.6], [W])

$Y$ has only canonical singularities. The dissident locus $\Sigma_{0}$ has codimension at 
least 4 in $Y$. The complement $\Sigma \backslash \Sigma_{0}$ is either empty or the disjoint union of codimension 2 smooth and symplectic subvarieties of $Y \backslash \Sigma_{0}$.

Keep the notation of Proposition 3.1. Let $[E]$ be the class of $E$ in $H^{2}(X, \mathbb{Z})$, and let $R$ be the reflection of $H^{2}(X, \mathbb{Q})$ given by

$$
R(x):=x-\left(\frac{2(x,[E])}{([E],[E])}\right)[E] .
$$

Consider the natural isomorphism $H^{2}(X, \mathbb{Z})^{*} \cong H_{2}(X, \mathbb{Z})$, given by the universal coefficients theorem and the fact that $X$ is simply connected. Denote by

$$
[E]^{\vee} \in H_{2}(X, \mathbb{Q})
$$

the class corresponding to $-2([E], \bullet) /([E],[E])$, where both pairings in the fraction are the Beauville-Bogomolov pairing.

We identify $H^{2}(X, \mathbb{Z})$ and $H^{2}\left(X^{\prime}, \mathbb{Z}\right)$ via the graph of the birational map. This graph induces a Hodge isometry, and the isometry maps the class $[E] \in$ $H^{2}(X, \mathbb{Z})$ to the class $\left[E^{\prime}\right] \in H^{2}\left(X^{\prime}, \mathbb{Z}\right)$, by [OG1, Proposition 1.6.2]. We get an identification of the dual groups $H_{2}(X, \mathbb{Z})$ and $H_{2}\left(X^{\prime}, \mathbb{Z}\right)$. The following corollary was proven, in the case where $E$ is an irreducible component of a contractible divisor, in [Ma5, Lemmas 4.10, 4.23]. We are now able to extend it to the more general case of a prime exceptional divisor $E$. The following is a corollary of Proposition 3.1, Proposition 3.5, and [Ma5, Lemmas 4.10, 4.23].

\section{COROLLARY 3.6}

(1) The class $[E]^{\vee} \in H_{2}(X, \mathbb{Z})$ corresponds to the class in $H_{2}\left(X^{\prime}, \mathbb{Z}\right)$ of the generic fiber of the contraction $E^{\prime} \rightarrow Y$ in Proposition 3.1. The generic fiber is either a smooth rational curve or the union of two homologous smooth rational curves meeting at one point. In particular, $[E]^{\vee}$ is an integral class in $H_{2}(X, \mathbb{Z})$, and $R$ is an integral isometry.

(2) The reflection $R$ is a monodromy operator in $\operatorname{Mon}^{2}(X)$ as well as $\operatorname{Mon}^{2}\left(X^{\prime}\right)$. $R$ preserves the Hodge structure. The action of $R$ on $H^{1,1}\left(X^{\prime}\right) \cong$ $H^{1}\left(X^{\prime}, T X^{\prime}\right)$ induces an involution of $\operatorname{Def}\left(X^{\prime}\right)$, which generates the Galois group of the double cover of the Kuranishi deformation spaces $\operatorname{Def}\left(X^{\prime}\right) \rightarrow \operatorname{Def}(Y)$.

(3) Either $[E]$ is a primitive class of $H^{2}(X, \mathbb{Z})$, or $[E]$ is twice a primitive class. Similarly, $[E]^{\vee}$ is either a primitive class or twice a primitive class. Finally, at least one of $[E]$ or $[E]^{\vee}$ is a primitive class.

\section{Proof}

(1) The singular locus of $Y$ contains a unique irreducible component $\Sigma$ of codimension 2 , and $Y$ has singularities of type $A_{1}$ or $A_{2}$ along the Zariski dense open subset $\Sigma \backslash \Sigma_{0}$, by Proposition 3.5 (see also the classification of singularities in [Na1, Section 1.8]). When $X=X^{\prime}$, the class $\left[E^{\prime}\right]^{\vee}$ is the class of the fiber of the composite morphism $E^{\prime} \hookrightarrow X^{\prime} \rightarrow Y$, by [Ma5, Lemmas 4.10, 4.23].

(2) $R$ is a monodromy operator in $\operatorname{Mon}^{2}\left(X^{\prime}\right)$, by [Ma5, Lemmas 4.10, 4.23]. Now the isometry $Z_{*}: H^{2}(X, \mathbb{Z}) \rightarrow H^{2}\left(X^{\prime}, \mathbb{Z}\right)$, induced by the graph $Z$ of the 
birational map, is a parallel transport operator. This follows from the proof of [Hu3, Theorem 2.5]. In this proof Huybrechts constructed a correspondence $\Gamma:=$ $Z+\sum_{i} Y_{i}$ in $X \times X^{\prime}$ with the following properties: $\Gamma_{*}: H^{2}(X, \mathbb{Z}) \rightarrow H^{2}\left(X^{\prime}, \mathbb{Z}\right)$ is a parallel transport operator, $Z$ is the closure of the graph of the birational map as above, and the image of $Y_{i}$ in each factor $X$ and $X^{\prime}$ has codimension $\geq 2$. It follows that the two isometries $Z_{*}$ and $\Gamma_{*}$ coincide.

(3) The statements about the divisibility of $[E]$ and $[E]^{\vee}$ follow from the equality $\int_{[E]^{\vee}}[E]=-2$.

We denote by

$$
e \in H^{2}(X, \mathbb{Z})
$$

the primitive class, such that either $[E]=e$ or $[E]=2 e$. Let $e^{\vee}$ be the primitive class in $H_{2}(X, \mathbb{Z})$, such that $[E]^{\vee}=e^{\vee}$ or $[E]^{\vee}=2 e^{\vee}$. The divisibility factor $\operatorname{div}(e, \bullet)$, of the class $(e, \bullet) \in H^{2}(X, \mathbb{Z})^{*}$, is the positive number satisfying the equality $(e, \bullet)=\operatorname{div}(e, \bullet) \cdot e^{\vee}$.

\section{LEMMA 3.7}

We have

$$
-\operatorname{div}(e, \bullet)= \begin{cases}(e, e) / 2 & \text { if }[E]=e \text { and }[E]^{\vee}=e^{\vee} \\ (e, e) & \text { if }[E]=2 e \text { and }[E]^{\vee}=e^{\vee} \\ (e, e) & \text { if }[E]=e \text { and }[E]^{\vee}=2 e^{\vee}\end{cases}
$$

Proof

Let $[E]=k_{1} e$ and $[E]^{\vee}=k_{2} e^{\vee}$. Then

$$
-(e, \bullet)=\frac{-1}{k_{1}}([E], \bullet)=\frac{([E],[E])}{2 k_{1}}[E]^{\vee}=\frac{k_{1}(e, e)}{2}[E]^{\vee}=\frac{k_{1} k_{2}(e, e)}{2} e^{\vee} .
$$

\section{REMARK 3.8}

Let $L$ be the line bundle with $c_{1}(L)=e$. Then $\operatorname{dim} H^{0}\left(X, L^{n}\right)$ is either 0 or 1 , for all $n \in \mathbb{Z}$, by [Bou, Proposition 3.13]. Hence, there exists at most one nonzero integer $n$, such that the linear system $\left|L^{n}\right|$ contains a prime divisor. In particular, for a given pair $(X, e)$, at most one of the equalities $\left([E],[E]^{\vee}\right)=\left(e, 2 e^{\vee}\right)$ or $\left([E],[E]^{\vee}\right)=\left(2 e, e^{\vee}\right)$ can hold, for some prime divisor $E$ with $[E] \in \operatorname{span}_{\mathbb{Z}}\{e\}$. The same holds for an exceptional divisor, where the coefficients $n_{i}$ in Definition 3.2 are integral and with $\operatorname{gcd}\left\{n_{i}: 1 \leq i \leq k\right\}=1$.

\section{Holomorphic symplectic manifolds of $K 3^{[n]}$-type}

We prove Proposition 1.5 in this section. This completes the proof of Theorem 1.2. The lattice $H^{2}(X, \mathbb{Z})$ has signature $(3,20)$ (see [Be1]). A 3-dimensional subspace of $H^{2}(X, \mathbb{R})$ is said to be positive definite, if the Beauville-Bogomolov pairing restricts to it as a positive-definite pairing. The unit 2 -sphere, in any positivedefinite 3 -dimensional subspace, is a deformation retract of the positive cone 
$\mathcal{C}_{+} \subset H^{2}(X, \mathbb{R})$, given by $\mathcal{C}_{+}:=\left\{\lambda \in H^{2}(X, \mathbb{R}):(\lambda, \lambda)>0\right\}$. Hence, $H^{2}\left(\mathcal{C}_{+}, \mathbb{Z}\right)$ is isomorphic to $\mathbb{Z}$ and is a natural representation of the isometry group $O H^{2}(X, \mathbb{R})$. We denote by $O_{+} H^{2}(X, \mathbb{Z})$ the index two subgroup of $O H^{2}(X, \mathbb{Z})$, which acts trivially on $H^{2}\left(\mathcal{C}_{+}, \mathbb{Z}\right)$.

Let $X$ be of $K 3^{[n]}$-type, $n \geq 2$. Embed the lattice $H^{2}(X, \mathbb{Z})$ in its dual lattice $H^{2}(X, \mathbb{Z})^{*}$, via the Beauville-Bogomolov form.

\section{THEOREM 4.1 ([Ma4, THEOREM 1.2, LEMMA 4.2])}

$\operatorname{Mon}^{2}(X)$ is equal to the subgroup of $O_{+} H^{2}(X, \mathbb{Z})$, which acts via multiplication by 1 or -1 on the quotient group $H^{2}(X, \mathbb{Z})^{*} / H^{2}(X, \mathbb{Z})$.

The quotient $H^{2}(X, \mathbb{Z})^{*} / H^{2}(X, \mathbb{Z})$ is a cyclic group of order $2 n-2$. Indeed, we may deform $X$ to the Hilbert scheme $S^{[n]}$ of length $n$ subschemes of a $K 3$ surface $S . H^{2}\left(S^{[n]}, \mathbb{Z}\right)$ is Hodge-isometric to the orthogonal direct sum

$$
H^{2}(S, \mathbb{Z}) \oplus \mathbb{Z} \delta
$$

where $\delta$ is half the class of the big diagonal, and $(\delta, \delta)=2-2 n$ (see [Be1]). Let $\pi: S^{[n]} \rightarrow S^{(n)}$ be the Hilbert-Chow morphism onto the symmetric product of $S$. The isometric embedding $H^{2}(S, \mathbb{Z}) \hookrightarrow H^{2}\left(S^{[n]}, \mathbb{Z}\right)$ is given by the composition $H^{2}(S, \mathbb{Z}) \cong H^{2}\left(S^{(n)}, \mathbb{Z}\right) \stackrel{\pi^{*}}{\longrightarrow} H^{2}\left(S^{[n]}, \mathbb{Z}\right)$.

\section{Proof of Proposition 1.5}

The lattice $H^{2}(X, \mathbb{Z})$ is isometric to the orthogonal direct sum (4.1). Let $e$ be a class in $H^{2}(X, \mathbb{Z})$ of negative Beauville-Bogomolov degree, and let $R_{e}(x):=$ $x-2 \frac{(x, e)}{(e, e)} e$ be the reflection by $e$. Then $R_{e}$ is an integral isometry of $H^{2}(X, \mathbb{Z})$, which acts by 1 or -1 on the quotient $H^{2}(X, \mathbb{Z})^{*} / H^{2}(X, \mathbb{Z})$, if and only if $e$ has one of the two properties in the statement of Proposition 1.5, by [GHS, Corollary 3.4]. The proposition now follows from Theorem 4.1.*

\section{REMARK 4.2}

Let $X$ be of $K 3^{[n]}$-type, let $L$ be a line bundle on $X$, and set $\alpha:=c_{1}(L)$. Then the sheaf-cohomology Euler characteristic of $L$ is given by the binomial coefficient $\chi(L)=([(\alpha, \alpha) / 2]+n+1)$ by [Hu2, Section 3.4, Example 7]. We get the following equalities:

$$
\begin{gathered}
\chi(L)= \begin{cases}1 & \text { if }(\alpha, \alpha)=-2, \\
0 & \text { if }(\alpha, \alpha)=2-2 n \text { and } n \geq 3,\end{cases} \\
\chi\left(L^{2}\right)=0 \quad \text { if }(\alpha, \alpha)=-2 \text { and } n \geq 3, \\
\chi\left(L^{2}\right)<0 \quad \text { if }(\alpha, \alpha)=2-2 n \text { and } n \geq 2 .
\end{gathered}
$$

${ }^{*}$ I thank V. Gritsenko for reference [GHS, Corollary 3.4], which drastically shortens the original proof. 


\section{Deformation equivalence}

This section is influenced by an early draft of a paper of Brendan Hassett and Yuri Tschinkel, [HT3], which was graciously communicated to the author. The results rely heavily on Verbitsky's Torelli theorem (Theorem 5.7).

\subsection{The prime exceptional property of pairs $(X, L)$ is open}

Let $X$ be a projective irreducible holomorphic symplectic manifold, and let $E$ be a prime exceptional divisor. Set $c:=[E] \in H^{2}(X, \mathbb{Z})$. Given a point $t \in \operatorname{Def}(X)$, let $c_{t} \in H^{2}\left(X_{t}, \mathbb{Z}\right)$ be the class associated to $c$ via the parallel transport isomorphism $^{*} H^{2}(X, \mathbb{Z}) \rightarrow H^{2}\left(X_{t}, \mathbb{Z}\right)$. Denote by $R_{c}$ both the reflection of $H^{2}(X, \mathbb{Z})$ with respect to $c$, as well as the involution of $\operatorname{Def}(X)$. Let $D_{c} \subset \operatorname{Def}(X)$ be the fixed locus of $R_{c}$. $D_{c}$ is a smooth divisor in $\operatorname{Def}(X)$, which is characterized also as the subset

$$
D_{c}:=\left\{t \in \operatorname{Def}(X): c_{t} \text { is of Hodge type }(1,1)\right\} .
$$

\section{LEMMA 5.1}

There exists an open subset $D_{c}^{0} \subset D_{c}$, containing 0 , such that for every $t \in D_{c}^{0}$ the class $c_{t}$ is Poincaré dual to a prime exceptional divisor $E_{t}$.

\section{Proof}

Let $X^{\prime}, E^{\prime}$, and $Y$ be as in Proposition 3.1. Denote the image of $E^{\prime} \rightarrow Y$ by $B$. The generic fiber of $E^{\prime} \rightarrow B$ is either a smooth rational curve $C$, whose normal bundle satisfies

$$
N_{C / X^{\prime}} \cong \omega_{C} \oplus\left(\bigoplus_{i=1}^{2 n-2} \mathcal{O}_{C}\right),
$$

or the union of two such curves meeting nontangentially at one point, by Proposition 3.5. Druel showed that the exceptional locus of the birational map $X \rightarrow X^{\prime}$ does not dominate $B$ (see the proof of $[\mathrm{D}$, Theorem 1.3]). We conclude that a Zariski dense open subset of the original divisor $E$ in $X$ is covered by such rational curves. The proposition now follows from results of Ziv Ran about the deformations of such pairs $(X, C)$ (see [R, Theorem 1], with further comments in $[\mathrm{Ka}])$. Our argument is inspired by [HT1, Theorems 4.1, 4.3]. Note first that the class of the curve $C$ remains of type $(n-1, n-1)$ over $D_{c}$, by Corollary 3.6(1). Let $\psi: \mathcal{X} \rightarrow D_{c}$ be the semiuniversal family, let $\mathcal{H} \rightarrow D_{c}$ be the irreducible component of the relative Douady space containing the point $t_{0}$ representing the pair $(X, C)$, and let $\mathcal{C} \subset \mathcal{H} \times{ }_{D_{c}} \mathcal{X}$ be the universal subscheme. We get the diagram

$$
\begin{array}{rrr}
\mathcal{C} & \stackrel{f}{\longrightarrow} & \mathcal{X} \\
\alpha \downarrow & \downarrow \psi \\
t_{0} \in \mathcal{H} \stackrel{\beta}{\longrightarrow} & D_{c}
\end{array}
$$

\footnotetext{
${ }^{*}$ The local system $R^{2} \psi_{*} \mathbb{Z}$ over $\operatorname{Def}(X)$ is trivial, since we may choose $\operatorname{Def}(X)$ to be simply connected.
} 
Let $\pi: H^{1}\left(C, N_{C / X}\right) \rightarrow H^{2 n}\left(\Omega_{X}^{2 n-2}\right)$ be the semiregularity map. Then $\pi$ is an isomorphism of these one-dimensional vector spaces (Observation (a) before Corollary 4 in $[R])$. Theorem 1 of $[R]$ implies that the morphism $\beta$ is surjective, of relative dimension $2 n-2$, and smooth at the point $t_{0}$. It follows that $\mathcal{C}$ has relative dimension $2 n-1$ over $D_{c}$, and $\mathcal{C}$ is smooth along the rational curve $C$ over $t_{0}$. Furthermore, the fiber $(\beta \circ \alpha)^{-1}(0)$ contains a unique irreducible component $\widetilde{E}$, which dominates $E$, and $f: \widetilde{E} \rightarrow E$ has degree 1, by Corollary 3.6(1).

We claim that the differential $d f: T \mathcal{C} \rightarrow f^{*} T \mathcal{X}$ is injective along $C . T \mathcal{C}$ comes with a natural filtration $T_{\alpha} \subset T_{\beta \circ \alpha} \subset T \mathcal{C} ;\left(f^{*} T \mathcal{X}\right)_{\left.\right|_{C}}$ comes with the filtration $T C \subset(T X)_{\left.\right|_{C}} \subset(T \mathcal{X})_{\left.\right|_{C}}$. The homomorphism $d f$ is compatible with the filtrations and induces the identity on the first and third graded summands $T C$ and $T_{0}\left(D_{c}\right)$. It suffices to prove injectivity on the middle graded summand. The above condition on $N_{C / X}$ implies, furthermore, that the evaluation homomorphism $H^{0}\left(N_{C / X}\right) \otimes \mathcal{O}_{C} \rightarrow N_{C / X}$ is injective. It follows that the differential $d f$ is injective along $C$. Consequently, $f(\mathcal{C})$ determines a divisor $\mathcal{E}$ in $\mathcal{X}$, possibly after eliminating embedded components of $f(\mathcal{C})$, which are disjoint from the curve $C$. Furthermore, $\mathcal{E}$ intersects the fiber $X$ of $\psi$ along a divisor $E_{0}$ containing $E$, and $E_{0}$ is reduced along $E$.

It remains to prove that $E_{0}$ is irreducible. Now the fiber $X_{t}$ has a cyclic Picard group, for a generic $t \in D_{c}$. Hence, the generic fiber $E_{t}$ of $\mathcal{E}$ is of class $k c_{t}$, for some positive integer $k$. Thus $E_{0}$ is of class $k c$. But the linear system $|k E|$ consists of a single divisor $k E$, by [Bou, Proposition 3.13]. We get that $k=1$, since $E_{0}$ is reduced along $E$.

Let $\pi: \mathcal{X} \rightarrow T$ be a smooth family of irreducible holomorphic symplectic manifolds over a connected complex manifold $T$. Assume that there exists a section $e$ of $R_{\pi_{*}}^{2} \mathbb{Z}$, everywhere of Hodge type $(1,1)$. Given a point $t \in T$, denote by $L_{t}$ the line bundle on the fiber $X_{t}$ with class $e_{t}$.

\section{PROPOSITION 5.2}

Assume given a point $0 \in T$, such that the fiber $X_{0}$ is projective and the linear system $\left|L_{0}^{k}\right|$, of the kth tensor power, consists of a prime exceptional divisor $E_{0} \subset X_{0}$, for some positive integer $k$. Then $k=1$ or 2 . Let $Z \subset T$ be the subset of points $t \in T$, such that $h^{0}\left(X_{t}, L_{t}^{k}\right)>1$, or there exists a nonprime divisor, which is a member of the linear system $\left|L_{t}^{k}\right|$. Then $Z$ is a proper and closed analytic subset of $T$. Furthermore, there exists an irreducible divisor $\mathcal{E}$ in $\mathcal{X} \backslash \pi^{-1}(Z)$, such that $\mathcal{E}$ intersects the fiber $\pi^{-1}(t)$ along a prime exceptional divisor $E_{t}$ of class $k e_{t}$, for every $t$ in the complement $T \backslash Z$.

Proof

The integer $k$ is 1 or 2 by Corollary 3.6. The dimension $h^{0}\left(X_{t}, L_{t}^{k}\right)$ is an uppersemicontinuous function on $T$, and so the locus where it is positive is a closed analytic subset of $T$. On the other hand, $h^{0}\left(X_{t}, L_{t}^{k}\right)$ is positive over an open 
subset, by Lemma 5.1. Hence, it is positive everywhere, and $L_{t}^{k}$ is effective for all $t$.

Let $Z_{1} \subset T$ be the closed analytic subset, where $h^{0}\left(X_{t}, L_{t}^{k}\right)>1$. We know that $h\left(X_{0}, L_{0}^{k}\right)=1$, by [Bou, Proposition 3.13]. Hence, we may assume, possibly after replacing $T$ by $T \backslash Z_{1}$, that $h^{0}\left(X_{t}, L_{t}^{k}\right)=1$, for all $t \in T$.

We prove next that the section $e$ lifts to a line bundle $\mathcal{L} \cong \mathcal{O}_{\mathcal{X}}(\mathcal{E})$, for a divisor $\mathcal{E} \subset \mathcal{X}$, which does not contain any fiber of $\pi$. The following is part of the edge exact sequence of the spectral sequence of the composite functor $\Gamma \circ \pi_{*}$ of pushforward and taking global sections:

$$
H^{1}\left(T, \mathcal{O}_{T}^{*}\right) \rightarrow H^{1}\left(\mathcal{X}, \mathcal{O}_{\mathcal{X}}^{*}\right) \rightarrow H^{0}\left(T, R_{\pi_{*}}^{1} \mathcal{O}_{\mathcal{X}}^{*}\right) \rightarrow H^{2}\left(T, \mathcal{O}_{T}^{*}\right) .
$$

Let $V$ be an open subset of $T$ satisfying $H^{i}\left(V, \mathcal{O}_{V}^{*}\right)=0$, for $i=1,2$. Then the restriction of $e$ to $V$ lifts to a line bundle $\mathcal{L}_{V}$ over $\pi^{-1}(V)$. Now $\pi_{*} \mathcal{L}_{V}$ is a line bundle over $V$, which must be trivial, by the vanishing of $H^{1}\left(V, \mathcal{O}_{V}^{*}\right)$. Hence, $H^{0}\left(\pi^{-1}(V), \mathcal{L}_{V}\right) \cong H^{0}\left(V, \mathcal{O}_{V}\right)$, and there exists a unique divisor $\mathcal{E}_{V} \subset \pi^{-1}(V)$, in the linear system $\left|\mathcal{L}_{V}\right|$, which does not contain any fiber of $\pi$. If $V_{1}$ and $V_{2}$ are two such open subsets of $T$, then the divisors $\mathcal{E}_{V_{i}}$ constructed above agree along $\pi^{-1}\left(V_{1} \cap V_{2}\right)$, since they are canonical over any subset $V$ of $V_{1} \cap V_{2}$, over which $H^{i}\left(V, \mathcal{O}_{V}^{*}\right)=0$, for $i=1,2$. Hence, we get a global divisor $\mathcal{E} \subset \mathcal{X}$. Set $\mathcal{L}:=\mathcal{O}_{\mathcal{X}}(\mathcal{E})$.

We prove next that $\mathcal{E}$ is irreducible. Let $p: \mathcal{E} \rightarrow T$ be the restriction of $\pi$. Then $p$ is a proper morphism, which is also flat by [Mat, Application 2, p. 150]. All fibers of $p$ are connected, since $T$ is smooth and in particular normal, and the fiber over 0 is connected. The morphism $p$ is smooth along the smooth locus of $E_{0}$, and $\mathcal{E}$ is a local complete intersection in the smooth complex manifold $\mathcal{X}$. Hence, there exists precisely one irreducible component of $\mathcal{E}$ which contains $E_{0}$. Assume that there exists another irreducible component $\mathcal{E}^{\prime}$. Then $\mathcal{E}^{\prime}$ maps to a proper closed subset of $T$, which does not contain 0 . But $T$ is irreducible, and $\mathcal{E}^{\prime}$ intersects each fiber of $\pi$ along a subset which is either empty or of codimension at least one. Hence, the codimension of $\mathcal{E}^{\prime}$ in $\mathcal{X}$ is larger than one. This contradicts the fact that $\mathcal{E}$ is a divisor. We conclude that $\mathcal{E}$ is irreducible.

The subset $Z \subset T$, consisting of points $t \in T$, where $E_{t}$ is reducible or nonreduced, is a closed analytic subset of $T$, which does not contain 0 .

Proposition 5.2 shows that the property that $L$ is prime exceptional is open in any smooth and connected base $T$ of a deformation of a pair $(X, L)$, as long as it holds for at least one projective pair. One limiting case is that of a pair $(X, L)$, where $L$ is exceptional, in the sense of Definition 3.2, but no longer prime. However, the exceptional property is unfortunately not closed, as the following example shows.

\section{EXAMPLE 5.3}

Let $Y$ be the intersection of a quadric and a cubic in $\mathbb{P}^{4}$, which are tangent at one point $y_{0}$, such that $Y$ has an ordinary double point at $y_{0}$. Let $H$ be the hyperplane line bundle on $Y . Y$ is a singular $K 3$ surface of degree 6 . Let 
$\pi: X \rightarrow Y$ be the blowup of $Y$ at $y_{0}$, and let $E \subset X$ be its exceptional divisor. $X$ is a smooth $K 3$ surface. Set $L_{0}:=\pi^{*} H \otimes \mathcal{O}_{X}(2 E)$. Then $L_{0}$ has degree -2 , but $L_{0}$ is not exceptional. Set $c:=c_{1}\left(L_{0}\right)$, let $D_{c} \subset \operatorname{Def}(X)$ be the divisor defined in equation (5.1), and denote by $L_{t}$ the line bundle on $X_{t}$ with class $c_{t}, t \in D_{c}$. Then $L_{t}$ has degree -2 , and thus precisely one of $L_{t}$ or $L_{t}^{-1}$ is effective (see [BHPV, Chapter VIII, Proposition 3.6]). The semicontinuity theorem implies that $L_{t}$ is effective, since $L_{0}^{-1}$ is not and $D_{c}$ is connected. For a generic $t \in D_{c}$, the pair $\left(X_{t}, L_{t}\right)$ consists of a Kähler $K 3$ surface, whose Picard group is generated by $L_{t}$. Hence, the linear system $\left|L_{t}\right|$ consists of a single smooth rational curve $E_{t}$. The analogue of Lemma 5.1 is known for such a pair $\left(X_{t}, L_{t}\right)$, even if $X_{t}$ is not projective. Hence, Proposition 5.2 applies as well. Let $D_{c}^{0} \subset D_{c}$ be the subset of pairs $\left(X_{t}, L_{t}\right)$, such that $L_{t} \cong \mathcal{O}_{X_{t}}\left(E_{t}\right)$, for a smooth connected rational curve $E_{t}$. We get that $D_{c}^{0}$ is nonempty and the complement $Z:=D_{c} \backslash D_{c}^{0}$ is a closed analytic subset containing $0 \in D_{c}$. Consequently, the property of $L_{t}$ being exceptional is not closed.

\subsection{Deformation equivalence and Torelli}

We introduce and relate three notions of deformation equivalence of pairs.

\section{DEFINITION 5.4}

Let $\left(X_{i}, E_{i}\right), i=1,2$, be two pairs of an irreducible holomorphic symplectic manifold $X_{i}$, and an effective divisor $E_{i} \in \operatorname{Div}\left(X_{i}\right)$. The two pairs are said to be deformation equivalent, if there exists a smooth proper family $\pi: \mathcal{X} \rightarrow T$ of irreducible holomorphic symplectic manifolds, over a connected analytic space $T$ with finitely many irreducible components, a holomorphic line bundle $\mathcal{L}$ over $\mathcal{X}$, a nowherevanishing section $s$ of $\pi_{*} \mathcal{L}$, points $t_{i} \in T$, and isomorphisms $f_{i}: \mathcal{X}_{t_{i}} \rightarrow X_{i}$, such that $f_{i}\left(\left(s_{t_{i}}\right)_{0}\right)=E_{i}, i=1,2$. Above $\left(s_{t_{i}}\right)_{0}$ denotes the zero divisor of $s_{t_{i}}$ in $X_{t_{i}}$.

The relation is clearly symmetric and reflexive. It is also transitive, since we allow $T$ to be reducible.

\section{DEFINITION 5.5}

Let $\left(X_{i}, L_{i}\right), i=1,2$, be two pairs of an irreducible holomorphic symplectic manifold $X_{i}$ and a line bundle $L_{i}$. The two pairs are said to be deformation equivalent if there exists a smooth proper family $\pi: \mathcal{X} \rightarrow T$ of irreducible holomorphic symplectic manifolds, over a connected analytic space $T$ with finitely many irreducible components, and a section $e$ of $R^{2} \pi_{*} \mathbb{Z}$, which is everywhere of Hodge-type $(1,1)$, points $t_{i} \in T$, and isomorphisms $f_{i}: \mathcal{X}_{t_{i}} \rightarrow X_{i}$, such that $\left(f_{i}\right)_{*}\left(e_{t_{i}}\right)=c_{1}\left(L_{i}\right)$.

\section{DEFINITION 5.6}

Let $\left(X_{i}, e_{i}\right), i=1,2$, be two pairs of an irreducible holomorphic symplectic manifold $X_{i}$ and a class $e_{i} \in H^{2}\left(X_{i}, \mathbb{Z}\right)$. The two pairs are said to be deformation equivalent if there exists a smooth proper family $\pi: \mathcal{X} \rightarrow T$ of irreducible holomorphic symplectic manifolds, over a connected analytic space $T$ with finitely 
many irreducible components, a section $e$ of $R^{2} \pi_{*} \mathbb{Z}$, points $t_{i} \in T$, and isomorphisms $f_{i}: \mathcal{X}_{t_{i}} \rightarrow X_{i}$, such that $\left(f_{i}\right)_{*}\left(e_{t_{i}}\right)=e_{i}$.

Definitions 5.5, 5.4, and 5.6, fit in a hierarchy. If $L_{i}=\mathcal{O}_{X_{i}}\left(E_{i}\right)$ and $e_{i}:=c_{1}\left(L_{i}\right)$, then

$$
\begin{gathered}
\left(X_{1}, E_{1}\right) \equiv\left(X_{2}, E_{2}\right) \Rightarrow\left(X_{1}, L_{1}\right) \equiv\left(X_{2}, L_{2}\right), \\
\left(X_{1}, L_{1}\right) \equiv\left(X_{2}, L_{2}\right) \Rightarrow\left(X_{1}, e_{1}\right) \equiv\left(X_{2}, e_{2}\right) .
\end{gathered}
$$

Assume that the divisors $E_{i}, i=1,2$, are prime exceptional, and assume that $X_{1}$ is projective. Then both implications above are equivalences, by Proposition 5.12. For a qualified "converse" to the second implication (5.2), without assuming that $L_{1}$ and $L_{2}$ are effective (see Lemma 5.17).

\subsubsection{Period maps}

A marking, for an irreducible holomorphic symplectic manifold $X$, is a choice of an isometry $\eta: H^{2}(X, \mathbb{Z}) \rightarrow \Lambda$ with a fixed lattice $\Lambda$. The period, of the marked manifold $(X, \eta)$, is the line $\eta\left[H^{2,0}(X)\right]$ considered as a point in the projective space $\mathbb{P}[\Lambda \otimes \mathbb{C}]$. The period lies in the period domain

$$
\Omega:=\{\ell:(\ell, \ell)=0 \text { and }(\ell, \bar{\ell})>0\} .
$$

$\Omega$ is an open subset, in the classical topology, of the quadric in $\mathbb{P}[\Lambda \otimes \mathbb{C}]$ of isotropic lines (see $[\mathrm{Be} 1]$ ).

There is a (non-Hausdorff) moduli space $\mathfrak{M}_{\Lambda}$ of marked irreducible holomorphic symplectic manifolds, with a second integral cohomology lattice isometric to $\Lambda$ (see $[\mathrm{Hu} 1])$. The period map

$$
\begin{aligned}
P: \mathfrak{M}_{\Lambda} & \longrightarrow \Omega, \\
(X, \eta) & \mapsto \eta\left[H^{2,0}(X)\right]
\end{aligned}
$$

is a local isomorphism, by the local Torelli theorem (see [Be1]). The surjectivity theorem states that the restriction of the period map to every connected component of $\mathfrak{M}_{\Lambda}$ is surjective (see [Hu1]).

Two points $x$ and $y$ of a topological space are inseparable if every pair of open subsets $U, V$, with $x \in U$ and $y \in V$, has a nonempty intersection. Assume given a bimeromorphic map $f: X_{1} \rightarrow X_{2}$ and a marking $\eta_{1}$ for $X_{1}$. Let $f^{*}$ : $H^{2}\left(X_{2}, \mathbb{Z}\right) \rightarrow H^{2}\left(X_{1}, \mathbb{Z}\right)$ be the isometry induced by the bimeromorphic map $f$ (see the proof of Corollary 3.6). Set $\eta_{2}=\eta_{1} \circ f^{*}$. Then $\left(X_{1}, \eta_{1}\right)$ and $\left(X_{2}, \eta_{2}\right)$ are inseparable points of $\mathfrak{M}_{\Lambda}$ (see [Hu3, Theorem 2.5]). Conversely, Verbitsky recently proved the following version of the Torelli theorem.

THEOREM 5.7 ([Ver, THEOREM 4.24], [Hu4])

Let $\mathfrak{M}_{\Lambda}^{0}$ be a connected component of $\mathfrak{M}_{\Lambda}$. If $\left(X_{1}, \eta_{1}\right)$ and $\left(X_{2}, \eta_{2}\right)$ are two pairs in $\mathfrak{M}_{\Lambda}^{0}$ and $P\left(X_{1}, \eta_{1}\right)=P\left(X_{2}, \eta_{2}\right)$, then $\left(X_{1}, \eta_{1}\right)$ and $\left(X_{2}, \eta_{2}\right)$ are inseparable points of $\mathfrak{M}_{\Lambda}^{0}$. 
A homomorphism $h: H^{*}\left(X_{1}, \mathbb{Z}\right) \rightarrow H^{*}\left(X_{2}, \mathbb{Z}\right)$ is a parallel transport operator if there exists a smooth and proper family $f: \mathcal{X} \rightarrow B$ of irreducible holomorphic symplectic manifolds over an analytic base $B$, points $b_{1}, b_{2}$ in $B$, isomorphisms $X_{i} \cong \mathcal{X}_{b_{i}}$, and a continuous path $\gamma$ from $b_{1}$ to $b_{2}$, such that parallel transport in the local system $R^{*} f_{*} \mathbb{Z}$ along $\gamma$ induces $h$. The following is a fundamental result of Huybrechts.

\section{THEOREM 5.8 ([Hu1, THEOREM 4.3])}

Let $\left(X_{1}, \eta_{1}\right)$ and $\left(X_{2}, \eta_{2}\right)$ be two inseparable points of $\mathfrak{M}_{\Lambda}$, with $\operatorname{dim}\left(X_{i}\right)=2 n$. Then there exists an effective cycle $\Gamma:=Z+\sum Y_{j}$ in $X_{1} \times X_{2}$, of pure dimension $2 n$, with the following properties.

(1) $Z$ is the graph of a bimeromorphic map from $X_{1}$ to $X_{2}$.

(2) The correspondence $[\Gamma]_{*}: H^{*}\left(X_{1}, \mathbb{Z}\right) \rightarrow H^{*}\left(X_{2}, \mathbb{Z}\right)$ is a parallel transport operator. Furthermore, the composition

$$
\eta_{2}^{-1} \circ \eta_{1}: H^{2}\left(X_{1}, \mathbb{Z}\right) \rightarrow H^{2}\left(X_{2}, \mathbb{Z}\right)
$$

is equal to the restriction of $[\Gamma]_{*}$.

(3) The image of the projection of each $Y_{j}$ into each $X_{i}, i=1,2$, has positive codimension in $X_{i}$.

Assume given two deformation equivalent pairs $\left(X_{i}, e_{i}\right), i=1,2$, in the sense of Definition 5.6. Then there exist isometries $\eta_{i}: H^{2}\left(X_{i}, \mathbb{Z}\right) \rightarrow \Lambda$, having the following two properties:

(1) $\eta_{1}\left(e_{1}\right)=\eta_{2}\left(e_{2}\right)$;

(2) the marked pairs $\left(X_{i}, \eta_{i}\right)$ belong to the same connected component $\mathfrak{M}_{\Lambda}^{0}$.

Let $\lambda$ be the common value $\eta_{i}\left(e_{i}\right), i=1,2$. If both classes $e_{i}$ belong to $H^{1,1}\left(X_{i}, \mathbb{Z}\right)$, then the periods $P\left(X_{i}, \eta_{i}\right)$ belong to the hyperplane $\lambda^{\perp} \subset \mathbb{P}[\Lambda \otimes \mathbb{C}]$ orthogonal to $\lambda$. The intersection $\lambda^{\perp} \cap \Omega$ is connected.

Fix a primitive nonzero class $\lambda \in \Lambda$ with $(\lambda, \lambda)<0$. Let

$$
\mathfrak{M}_{\Lambda, \lambda}^{0} \subset \mathfrak{M}_{\Lambda}^{0}
$$

be the subset parameterizing marked pairs $(X, \eta)$, such that $\eta^{-1}(\lambda)$ is of Hodge type $(1,1)$, and $\left(\kappa, \eta^{-1}(\lambda)\right)>0$, for some Kähler class $\kappa$ on $X$.

\section{CLAIM 5.9}

$\mathfrak{M}_{\Lambda, \lambda}^{0}$ is an open subset of $P^{-1}\left(\lambda^{\perp} \cap \Omega\right)$.

\section{Proof}

Let $\mathfrak{M}_{+}^{0}$ be the subset of $\mathfrak{M}_{\Lambda}^{0}$ consisting of marked pairs $(X, \eta)$, such that $\left(\kappa, \eta^{-1}(\lambda)\right)>0$, for some Kähler class $\kappa$ on $X$. It suffices to prove that $\mathfrak{M}_{+}^{0}$ is an open subset of $\mathfrak{M}_{\Lambda}^{0}$. Let $\left(X_{0}, \eta_{0}\right)$ be a point of $\mathfrak{M}_{+}^{0}$, and let $\kappa_{0}$ be a Kähler class on $X_{0}$ satisfying $\left(\kappa_{0}, \eta_{0}^{-1}(\lambda)\right)>0$. Let $\operatorname{Def}\left(X_{0}\right)$ be the Kuranishi deformation space, and let $\psi: \mathcal{X} \rightarrow \operatorname{Def}\left(X_{0}\right)$ be the semiuniversal family with fiber $X_{0}$ over $0 \in \operatorname{Def}\left(X_{0}\right)$. There exists an open subset $U$ of $\operatorname{Def}\left(X_{0}\right)$, and a differen- 
tiable section $\kappa$ of the real vector bundle $\left(R_{\psi_{*}}^{2} \mathbb{R}\right)_{\left.\right|_{U}}$, over $U$, such that $\kappa_{t}$ is a Kähler class of $X_{t}$, for all $t \in U$, by the proof of the stability of Kähler manifolds (see [Voi, Theorem 9.3.3]). We may identify $U$ with an open subset of $\mathfrak{M}_{\Lambda}^{0}$ containing $\left(X_{0}, \eta_{0}\right)$, by the local Torelli theorem. We get the continuous function $\left(\kappa_{t}, \eta_{t}^{-1}(\lambda)\right)$ over $U$, which is positive at $\left(X_{0}, \eta_{0}\right)$. Hence, there is an open subset $W \subset U$, containing $\left(X_{0}, \eta_{0}\right)$, such that $\left(\kappa_{t}, \eta_{t}^{-1}(\lambda)\right)>0$, for all $t \in W$.

The local Torelli theorem implies that the period map restricts to a local isomorphism

$$
P_{\lambda}: \mathfrak{M}_{\Lambda, \lambda}^{0} \longrightarrow \lambda^{\perp} \cap \Omega .
$$

$\mathfrak{M}_{\Lambda, \lambda}^{0}$ is thus a non-Hausdorff smooth complex manifold of dimension $b_{2}(X)-3$.

\subsection{2. $\mathfrak{M}_{\Lambda, \lambda}^{0}$ is path connected}

Given a point $t \in \Omega$, set $\Lambda_{t}^{1,1}:=\{x \in \Lambda:(x, t)=0\}$. The following statement is a Corollary of Theorem 5.7.

\section{COROLLARY 5.10}

Let $t \in \lambda^{\perp} \cap \Omega$ be a point, such that $\Lambda_{t}^{1,1}=\operatorname{span}_{\mathbb{Z}}\{\lambda\}$. Then the fiber $P_{\lambda}^{-1}(t)$ consists of the isomorphism class of a single marked pair.

\section{Proof}

Let $(X, \eta)$ be a marked pair in $P_{\lambda}^{-1}(t)$. Set $\tilde{\lambda}:=\eta^{-1}(\lambda)$. Then $H^{1,1}(X, \mathbb{Z})$ is spanned by $\tilde{\lambda}$, and there exists a Kähler class $\kappa_{0}$, such that $\left(\kappa_{0}, \tilde{\lambda}\right)>0$. Let us first describe the three possibilities for the Kähler cone $\mathcal{K}_{X}$ and the birational Kähler cone $\mathcal{B K}_{X}$ of $X$. Recall that $\mathcal{B} \mathcal{K}_{X}$ is the union of the subsets $f^{*}\left(\mathcal{K}_{Y}\right) \subset H^{1,1}(X, \mathbb{R})$, as $f$ varies over all bimeromorphic maps $f: X \rightarrow Y$ from $X$ to another irreducible holomorphic symplectic manifold $Y$. Denote by $\tilde{\lambda}^{\vee}$ the primitive class in $H^{2}(X, \mathbb{Z})^{*}$, which is a positive multiple of $(\tilde{\lambda}, \bullet)$. Let $\mathcal{C}_{X}$ be the connected component of the cone $\left\{\kappa \in H^{1,1}(X, \mathbb{R}):(\kappa, \kappa)>0\right\}$, which contains the Kähler cone.

Case 1. If $d \lambda^{\vee}$ is not represented by a rational curve, for any positive integer $d$, then $\mathcal{B K}_{X}=\mathcal{K}_{X}=\mathcal{C}_{X}$, by [Hu3, Corollary 3.3].

Case 2. Assume that $\tilde{\lambda}$ is $\mathbb{Q}$-effective. Then $d \tilde{\lambda}$ is represented by a prime exceptional divisor $E \subset X$, for some positive integer $d$, which is uniruled, by [Bou, Proposition 4.7]. Then

$$
\mathcal{B} \mathcal{K}_{X}=\mathcal{K}_{X}=\left\{\kappa \in \mathcal{C}_{X}:(\kappa, \tilde{\lambda})>0\right\},
$$

by [Bou, Theorem 4.3].

Case 3. Assume that $d \tilde{\lambda}$ is not effective, for any positive integer $d$, but $d \tilde{\lambda}^{\vee}$ is represented by a rational curve, for some positive integer $d$. Then

$$
\begin{aligned}
\mathcal{K}_{X} & =\left\{\kappa \in \mathcal{C}_{X}:(\kappa, \tilde{\lambda})>0\right\}, \\
\mathcal{B} \mathcal{K}_{X} & =\mathcal{K}_{X} \cup \mathcal{K}_{X}^{\prime}, \quad \text { where } \mathcal{K}_{X}^{\prime}=\left\{\kappa \in \mathcal{C}_{X}:(\kappa, \tilde{\lambda})<0\right\},
\end{aligned}
$$

by [Bou, Theorem 4.3]. 
Let $\left(X_{1}, \eta_{1}\right)$ and $\left(X_{2}, \eta_{2}\right)$ be two marked pairs in $P_{\lambda}^{-1}(t)$. Then $\left(X_{1}, \eta_{1}\right)$ and $\left(X_{2}, \eta_{2}\right)$ are inseparable points, by Theorem 5.7. Hence, there exists a cycle $\Gamma:=Z+\sum Y_{j}$ in $X_{1} \times X_{2}$, satisfying the properties listed in Theorem 5.8. Denote by $g: X_{1} \rightarrow X_{2}$ the bimeromorphic map with graph $Z$, and let $f: H^{2}\left(X_{1}, \mathbb{Z}\right) \rightarrow$ $H^{2}\left(X_{2}, \mathbb{Z}\right)$ be the parallel-transport operator $[\Gamma]_{*}$, so that $f=\eta_{2}^{-1} \circ \eta_{1}$, by Theorem 5.8. Set $\tilde{\lambda}_{i}:=\eta_{i}^{-1}(\lambda)$. Let $\kappa_{i}$ be a Kähler class on $X_{i}$, such that $\left(\tilde{\lambda}_{i}, \kappa_{i}\right)>0$.

In cases 1 and $3, X_{i}$ does not contain any effective divisor, $i=1,2$. In particular, the image of each $Y_{j}$ has codimension $\geq 2$ in each $X_{i}$, and $f=g_{*}$. We have $\left(\tilde{\lambda}_{1}, g^{*}\left(\kappa_{2}\right)\right)=\left(\eta_{1}^{-1}(\lambda), g^{*}\left(\kappa_{2}\right)\right)=\left(g_{*} \eta_{1}^{-1}(\lambda), \kappa_{2}\right)=\left(\eta_{2}^{-1}(\lambda), \kappa_{2}\right)=\left(\tilde{\lambda}_{2}, \kappa_{2}\right)$, since $g^{*}$ is an isometry. We conclude the inequality

$$
\left(\tilde{\lambda}_{1}, g^{*}\left(\kappa_{2}\right)\right)>0 .
$$

If $g^{*}\left(\kappa_{2}\right)$ is not a Kähler class, then the birational Kähler cone $\mathcal{B K}_{X_{1}}$ consists of at least two connected components. Thus we must be in case 3 . So $\kappa_{1} \in \mathcal{K}_{X_{1}}$ and $g^{*}\left(\kappa_{2}\right)$ belongs to $\mathcal{K}_{X_{1}}^{\prime}$. Hence, $\left(g^{*}\left(\kappa_{2}\right), \tilde{\lambda}_{1}\right)<0$, by the characterization of $\mathcal{K}_{X_{1}}^{\prime}$. This contradicts inequality (5.5).

We conclude that $g^{*}\left(\kappa_{2}\right)$ is a Kähler class. Thus $g$ is an isomorphism, by [Hu3, Proposition 2.1], and $\left(X_{1}, \eta_{1}\right)$ and $\left(X_{2}, \eta_{2}\right)$ are isomorphic marked pairs.

It remains to treat case 2 . In that case $\mathcal{B} \mathcal{K}_{X_{i}}=\mathcal{K}_{X_{i}}$, and so $g$ is an isomorphism. Hence, $g_{*}\left(\tilde{\lambda}_{1}\right)=\tilde{\lambda}_{2}$, since the classes $\tilde{\lambda}_{i}$ are effective. On the other hand, $f\left(\tilde{\lambda}_{1}\right)=\eta_{2}^{-1} \eta_{1}\left(\eta_{1}^{-1}(\lambda)\right)=\eta_{2}^{-1}(\lambda)=\tilde{\lambda}_{2}$. Hence, $f\left(\tilde{\lambda}_{1}\right)=g_{*}\left(\tilde{\lambda}_{1}\right)$. The subspace $\tilde{\lambda}_{1}^{\perp}$, orthogonal to $\tilde{\lambda}_{1}$, is necessarily in the kernel of the correspondence $\left[\sum Y_{j}\right]_{*}: H^{2}\left(X_{1}, \mathbb{Z}\right) \rightarrow H^{2}\left(X_{2}, \mathbb{Z}\right)$. Hence, $f(\alpha)=g_{*}(\alpha)$, for all $\alpha \in \tilde{\lambda}_{1}^{\perp}$. We conclude that $f=g_{*}$, and the two pairs $\left(X_{1}, \eta_{1}\right)$ and $\left(X_{2}, \eta_{2}\right)$ are isomorphic.

\section{COROLLARY 5.11}

$\mathfrak{M}_{\Lambda, \lambda}^{0}$ is a path-connected subset of $\mathfrak{M}_{\Lambda}^{0}$.

\section{Proof}

Let $(X, \eta)$ be a marked pair in $\mathfrak{M}_{\Lambda, \lambda}^{0}$. Then there exists a continuous path from $(X, \eta)$ to some $\left(X_{0}, \eta_{0}\right)$, where $H^{1,1}\left(X_{0}, \mathbb{Z}\right)$ is spanned by $\eta_{0}^{-1}(\lambda)$, by the local Torelli theorem. Hence, it suffices to construct a continuous path between any two pairs $\left(X_{0}, \eta_{0}\right)$ and $\left(X_{1}, \eta_{1}\right)$ in $\mathfrak{M}_{\Lambda, \lambda}^{0}$, such that $H^{1,1}\left(X_{i}, \mathbb{Z}\right)$ is cyclic, for $i=0,1$. Set $t_{i}:=P\left(X_{i}, \eta_{i}\right), i=0,1$. Let $I$ be the closed interval $[0,1]$. Let $\gamma: I \rightarrow \lambda^{\perp} \cap \Omega$ be a continuous path from $t_{0}$ to $t_{1}$. Let $I_{1} \subset I$ be the subset of points $t$, such that $\Lambda_{\gamma(t)}^{1,1}$ is cyclic. We may choose $\gamma$ so that $I_{1}$ is a dense subset of $I$.

For each $t \in I_{1}$, there exists a unique isomorphism class of a marked pair $\left(X_{t}, \eta_{t}\right)$ in $\mathfrak{M}_{\Lambda, \lambda}^{0}$ with period $\gamma(t)$, by Corollary 5.10 . Choose an open pathconnected subset $U_{t} \subset \mathfrak{M}_{\Lambda, \lambda}^{0}$, containing $\left(X_{t}, \eta_{t}\right)$, such that $P_{\lambda}$ restricts to $U_{t}$ as an open embedding. This is possible, by the local Torelli theorem. We get the open covering $\gamma(I) \subset \bigcup_{t \in I_{1}} P_{\lambda}\left(U_{t}\right)$. Choose a finite subcovering $\bigcup_{j=0}^{N} P_{\lambda}\left(U_{t_{j}}\right)$ of $\gamma(I)$, with $0=t_{0}<t_{1}<\cdots<t_{N}=1$. Choose an increasing subsequence $\tau_{j}:=t_{i_{j}}, 0 \leq$ $j \leq k$, such that $\tau_{0}=t_{0}, \tau_{k}=t_{N}$, and $P_{\lambda}\left(U_{\tau_{j}}\right) \cap P_{\lambda}\left(U_{\tau_{j+1}}\right)$ is nonempty. Choose points $s_{j, j+1}$ in $P_{\lambda}\left(U_{\tau_{j}}\right) \cap P_{\lambda}\left(U_{\tau_{j+1}}\right)$, such that $\Lambda_{s_{j, j+1}}^{1,1}$ is cyclic, and let $\tilde{s}_{j, j+1}$ be 
the unique point of $\mathfrak{M}_{\Lambda, \lambda}^{0}$ over $s_{j, j+1}$. Then $\tilde{s}_{j, j+1}$ belongs to $U_{\tau_{j}} \cap U_{\tau_{j+1}}$. Choose continuous paths $\alpha_{j}$ in $U_{\tau_{j}}$ from $\tilde{s}_{j-1, j}$ to $\left(X_{\tau_{j}}, \eta_{\tau_{j}}\right)$, if $j>0$, and $\beta_{j}$ in $U_{\tau_{j}}$ from $\left(X_{\tau_{j}}, \eta_{\tau_{j}}\right)$ to $\tilde{s}_{j, j+1}$, if $j<k$. Then the concatenated path $\beta_{0} \alpha_{1} \beta_{1} \cdots \alpha_{k-1} \beta_{k-1} \alpha_{k}$ is a continuous path from the isomorphism class of $\left(X_{0}, \eta_{0}\right)$ to that of $\left(X_{1}, \eta_{1}\right)$.

\subsubsection{Deformation equivalent monodromy-reflective line bundles are simultane-} ously stably $\mathbb{Q}$-effective or not stably $\mathbb{Q}$-effective

\section{PROPOSITION 5.12}

Let $\left(X_{1}, E_{1}\right),\left(X_{2}, E_{2}\right)$ be two pairs of irreducible holomorphic symplectic manifolds $X_{i}$ and prime exceptional divisors $E_{i} \subset X_{i}$. Assume that $X_{1}$ is projective and $\left(X_{1},\left[E_{1}\right]\right)$ is deformation equivalent to $\left(X_{2},\left[E_{2}\right]\right)$, in the sense of Definition 5.6. Then $\left(X_{1}, E_{1}\right)$ and $\left(X_{2}, E_{2}\right)$ are deformation equivalent in the sense of Definition 5.4 .

\section{Proof}

We assume, for simplicity of notation, that the class $\left[E_{i}\right]$ is primitive. The generalization of the proof to the case $\left[E_{i}\right]=2 e_{i}$ is straightforward. As noted above, we can choose a marking $\eta_{2}$ of $X_{2}$, such that $\left(X_{2}, \eta_{2}\right)$ belongs to $\mathfrak{M}_{\Lambda}^{0}$ and $\eta_{2}\left(\left[E_{2}\right]\right)=\lambda$. Any Kähler class $\kappa$ on $X_{2}$ satisfies $\left(\kappa,\left[E_{2}\right]\right)>0$, since $E_{2}$ is effective (see $[\mathrm{Hu} 1])$. Hence, $\left(X_{2}, \eta_{2}\right)$ belongs to $\mathfrak{M}_{\Lambda, \lambda}^{0}$.

Choose a continuous path $\gamma:[0,1] \rightarrow \mathfrak{M}_{\Lambda, \lambda}^{0}$ from $\left(X_{1}, \eta_{1}\right)$ to $\left(X_{2}, \eta_{2}\right)$. Further choose a sufficiently fine partition of the unit interval

$$
0=t_{0}<t_{1}<\cdots<t_{N}=1
$$

and open connected subsets $U_{i} \subset \mathfrak{M}_{\Lambda, \lambda}^{0}, 1 \leq i \leq N$, such that $\gamma\left(\left[t_{i-1}, t_{i}\right]\right)$ is contained in $U_{i}$, and the restriction of $P$ to $U_{i}$ is an open embedding $P_{i}: U_{i} \hookrightarrow$ $\lambda^{\perp} \cap \Omega$. This is possible by the local Torelli theorem.

\section{CLAIM 5.13}

For each $1 \leq i \leq N-1$, there exists a marked pair $\left(Y_{i}, \varphi_{i}\right)$ in $U_{i} \cap U_{i+1}$, such that $Y_{i}$ is projective, and $\varphi_{i}^{-1}(\lambda)$ is the class of a prime exceptional divisor on $Y_{i}$.

\section{Proof}

Following is an iterative process of constructing the pairs $\left(Y_{i}, \varphi_{i}\right)$. Set $\left(Y_{0}, \varphi_{0}\right)=$ $\left(X_{1}, \eta_{1}\right)$. Assume that $i=1$, or assume that $1<i \leq N-1$ and $\left(Y_{j}, \varphi_{j}\right)$ exists for all $1 \leq j<i$. The pair $\left(Y_{i-1}, \varphi_{i-1}\right)$ belongs to $U_{i}$. Proposition 5.2 implies that there exists a closed analytic subvariety $Z_{i} \subset U_{i}$, not containing $\left(Y_{i-1}, \varphi_{i-1}\right)$, such that for every $(X, \eta)$ in $U_{i} \backslash Z_{i}, \eta^{-1}(\lambda)$ is the class of a prime exceptional divisor $E \subset X$. The locus of projective marked pairs is dense in $U_{i} \cap U_{i+1}$, by [Hu2, Proposition 21]. Hence, there exists a projective pair $\left(Y_{i}, \varphi_{i}\right)$ in $\left[U_{i} \backslash Z_{i}\right] \cap$ $U_{i+1}$. 
Set $\left(Y_{N}, \eta_{N}\right):=\left(X_{2}, \eta_{2}\right)$. Let $D_{i} \subset Y_{i}$ be the prime exceptional divisor with $\left[D_{i}\right]=\eta_{i}^{-1}(\lambda)$. It remains to prove that $\left(Y_{i-1}, D_{i-1}\right)$ is deformation equivalent to $\left(Y_{i}, D_{i}\right)$, for $1 \leq i \leq N$. Both pairs $\left(Y_{i-1}, \varphi_{i-1}\right)$ and $\left(Y_{i}, \varphi_{i}\right)$ belong to $U_{i} \backslash Z_{i}$ by construction, for $i<N$, and by the characterization of $Z_{N}$ in Proposition 5.2, for $i=N$. Proposition 5.2 exhibits a divisor $\mathcal{E}_{i}$ in the restriction of $\mathcal{X}$ to $U_{i} \backslash Z_{i}$, whose fiber over the pair $\left(Y_{i}, \varphi_{i}\right)$ is $D_{i}$, and whose fiber over the pair $\left(Y_{i-1}, \varphi_{i-1}\right)$ is $D_{i-1}$. This completes the proof of Proposition 5.12.

The following variant of Proposition 5.12 will be used in the derivation of Theorem 1.11 from Torelli.

\section{PROPOSITION 5.14}

Let $X$ and $Y$ be two irreducible holomorphic symplectic manifolds, with $X$ projective, $E \subset X$ a prime exceptional divisor, and $L$ a line bundle on $Y$. Set $c:=c_{1}(L)$. Assume that $(X,[E])$ and $(Y, c)$ are deformation equivalent in the sense of Definition 5.6. Assume further that there exists a Kähler class $\kappa$ on $Y$, such that $(\kappa, c)>0$. Then $L$ is stably prime exceptional (in the sense of Theorem 1.11).

The above proposition was proven in the course of proving Proposition 5.12.

\section{COROLLARY 5.15}

Let $\left(X_{1}, L_{1}\right)$ and $\left(X_{2}, L_{2}\right)$ be two pairs, each of an irreducible holomorphic symplectic manifold $X_{i}$ and a monodromy-reflective line bundle $L_{i}$. Set $e_{i}:=c_{1}\left(L_{i}\right)$. Assume that $X_{1}$ is projective, $k e_{1}$ is the class of a prime exceptional divisor $E_{1}$, for some nonzero integer $k$, and $H^{0}\left(X_{2}, L_{2}^{d}\right)$ vanishes, for all nonzero integers $d$. Then the pairs $\left(X_{1}, e_{1}\right)$ and $\left(X_{2}, e_{2}\right)$ are not deformation equivalent, in the sense of Definition 5.6.

Proof

If $\left(X_{1}, e_{1}\right)$ and $\left(X_{2}, e_{2}\right)$ were deformation equivalent, in the sense of Definition 5.6, then $H^{0}\left(X_{2}, L_{2}^{d}\right)$ would not vanish for $d=k$ or $d=-k$, by Proposition 5.14 and the semicontinuity theorem.

\subsection{Deformation equivalence and monodromy invariants}

Let $\operatorname{Mon}^{2}(X)$ be the monodromy group, introduced in Definition 1.3. Let $I(X) \subset$ $H^{2}(X, \mathbb{Z})$ be a $\operatorname{Mon}^{2}(X)$-invariant subset, and let $\Sigma$ be a set.

\section{DEFINITION 5.16}

A function $f: I(X) \rightarrow \Sigma$ is a monodromy invariant, if $f(e)=f(g(e))$, for all $g \in \operatorname{Mon}^{2}(X)$. The function $f$ is said to be a faithful monodromy invariant if the function $\bar{f}: I(X) / \operatorname{Mon}^{2}(X) \rightarrow \Sigma$, induced by $f$, is injective.

Given an irreducible holomorphic symplectic manifold $X^{\prime}$, deformation equivalent to $X$, denote by $I\left(X^{\prime}\right) \subset H^{2}\left(X^{\prime}, \mathbb{Z}\right)$ the set of all classes $e^{\prime}$, such that 
$\left(X^{\prime}, e^{\prime}\right)$ is deformation equivalent to $(X, e)$, for some $e \in I(X)$, in the sense of Definition 5.6.

Assume that $f: I(X) \rightarrow \Sigma$ is a monodromy-invariant function. Then $f$ admits a natural extension to a function $f: I\left(X^{\prime}\right) \rightarrow \Sigma$, for every irreducible holomorphic symplectic manifold $X^{\prime}$ deformation equivalent to $X$. The extension is uniquely determined by the following condition. Given any smooth and proper family $\pi: \mathcal{X} \rightarrow T$, of irreducible holomorphic symplectic manifolds deformation equivalent to $X$, and any flat section e of the local system $R^{2} \pi_{*} \mathbb{Z}$, the function $f(e)$ is locally constant, in the classical topology of the analytic space $T$. We denote this extension by $f$ as well. The following statement relates monodromy invariants to deformation equivalence.

LEMMA 5.17

Let $f: I(X) \rightarrow \Sigma$ be a faithful monodromy-invariant function. Assume given two pairs $\left(X_{i}, e_{i}\right), i=1,2$, with $X_{i}$ deformation equivalent to $X$ and $e_{i} \in I\left(X_{i}\right)$.

(1) We have $f\left(e_{1}\right)=f\left(e_{2}\right)$ if and only if $\left(X_{1}, e_{1}\right)$ and $\left(X_{2}, e_{2}\right)$ are deformation equivalent, in the sense of Definition 5.6.

(2) Assume that $f\left(e_{1}\right)=f\left(e_{2}\right), e_{i}=c_{1}\left(L_{i}\right)$, for holomorphic line bundles $L_{i}$ on $X_{i}$, and there exist Kähler classes $\kappa_{i}$ on $X_{i}$, satisfying $\left(\kappa_{i}, e_{i}\right)>0$, for $i=1,2$. Then $\left(X_{1}, L_{1}\right)$ is deformation equivalent to $\left(X_{2}, L_{2}\right)$, in the sense of Definition 5.5 .

Proof

Part (1) is evident. Part (2) follows from part (1) and Corollary 5.11.

\section{Monodromy invariants from Mukai's isomorphism}

Let $S$ be a $K 3$ surface, and let $M$ be a smooth and projective moduli space of stable coherent sheaves on $S$. In Section 2.1 we recalled Mukai's embedding $\theta^{-1}: H^{2}(M, \mathbb{Z}) \rightarrow K(S)$, of the second cohomology of $M$, as a sublattice of the Mukai lattice. In Section 6.1 we use this embedding to define a monodromy invariant of a class in $H^{2}(M, \mathbb{Z})$. The values of this monodromy invariant, for monodromy-reflective classes, are calculated in Sections 6.2 and 7.

\subsection{A rank two sublattice of the Mukai lattice}

Let $\widetilde{\Lambda}$ be the unimodular lattice $E_{8}(-1)^{\oplus 2} \oplus U^{\oplus 4}$, where $U$ is the rank two unimodular hyperbolic lattice. $\widetilde{\Lambda}$ is isometric to the Mukai lattice of a $K 3$ surface. Let $X$ be an irreducible holomorphic symplectic manifold of $K 3^{[n]}$-type, $n \geq 2$. Choose an embedding $\iota: H^{2}(X, \mathbb{Z}) \hookrightarrow \widetilde{\Lambda}$ in the canonical $O(\widetilde{\Lambda})$-orbit of $X$ provided by Theorem 1.6. Let $v$ be a generator of the rank 1 sublattice of $\widetilde{\Lambda}$ orthogonal to the image of $\iota$. Then $(v, v)=2 n-2$. Let $e$ be a primitive class in $H^{2}(X, \mathbb{Z})$ satisfying $(e, e)=2-2 n$. We get the sublattice

$$
L:=\operatorname{span}_{\mathbb{Z}}\{e, v\} \subset \widetilde{\Lambda},
$$


where we denote by $e$ also the element $\iota(e)$. Let

$$
\widetilde{L}
$$

be the saturation of $L$ in $\widetilde{\Lambda}$. Note that the pair $(\widetilde{L}, e)$ determines the lattice $L$ via the equality $L=\mathbb{Z} e+\left[e^{\perp} \cap \widetilde{L}\right]$.

\section{DEFINITION 6.1}

Two pairs $\left(L_{i}, e_{i}\right), i=1,2$, each consisting of a lattice $L_{i}$ and a class $e_{i} \in L_{i}$, are said to be isometric, if there exists an isometry $g: L_{1} \rightarrow L_{2}$, such that $g\left(e_{1}\right)=e_{2}$.

\section{REMARK 6.2}

Let $L_{0}$ be a lattice. The set of isometry classes of pairs $\left(L_{1}, e_{1}\right)$, with $L_{1}$ isometric to $L_{0}$, is in natural bijection with the orbit set $L_{0} / O\left(L_{0}\right)$. The bijection sends the isometry class of $\left(L_{1}, e_{1}\right)$ to the orbit $O\left(L_{0}\right) g\left(e_{1}\right)$, where $g: L_{1} \rightarrow L_{0}$ is some isometry. The orbit $O\left(L_{0}\right) g\left(e_{1}\right)$ is independent of the choice of $g$.

Let $U$ be the rank 2 even unimodular hyperbolic lattice. Let $U(2)$ be the rank 2 lattice with Gram matrix $\left(\begin{array}{cc}0 & -2 \\ -2 & 0\end{array}\right)$. Let $H_{e v}$ be the rank 2 lattice with Gram matrix $\left(\begin{array}{cc}2 & 0 \\ 0 & -2\end{array}\right)$. Let $I_{n}^{\prime \prime}(X) \subset H^{2}(X, \mathbb{Z})$ be the subset of primitive classes of degree $2-2 n$, such that $\operatorname{div}(e, \bullet)=n-1$ or $\operatorname{div}(e, \bullet)=2 n-2$. Let $I_{n}(\widetilde{L}) \subset \widetilde{L}$ be the subset of primitive classes of degree $2-2 n$. Let $\rho$ be the largest positive integer, such that $(e+v) / \rho$ is an integral class. Define the integer $\sigma$ similarly using $(e-v)$.

\section{PROPOSITION 6.3}

(1) The isometry class of the lattice $\widetilde{L}$ is determined as follows.

$$
\widetilde{L} \cong \begin{cases}U & \text { if } \operatorname{div}(e, \bullet)=2 n-2, \\ H_{e v} & \text { if } \operatorname{div}(e, \bullet)=n-1 \text { and } n \text { is even, } \\ U(2) & \text { if } \operatorname{div}(e, \bullet)=n-1 \text { and } n \text { is odd }, n \neq 1(\bmod 8), \\ U(2) & \text { if } \operatorname{div}(e, \bullet)=n-1, n \equiv 1(\bmod 8) \text { and } \rho \sigma=2 n-2, \\ H_{e v} & \text { if } \operatorname{div}(e, \bullet)=n-1, n \equiv 1(\bmod 8) \text { and } \rho \sigma=n-1 .\end{cases}
$$

(2) Consider the function

$$
f: I_{n}^{\prime \prime}(X) \longrightarrow I_{n}(U) / O(U) \cup I_{n}(U(2)) / O(U(2)) \cup I_{n}\left(H_{e v}\right) / O\left(H_{e v}\right),
$$

which sends the pair $(X, e), e \in I_{n}^{\prime \prime}(X)$, to the isometry class of the pair $(\widetilde{L}, e)$, consisting of the primitive sublattice $\widetilde{L} \subset \widetilde{\Lambda}$, given in equation (6.1), and the class $e \in I_{n}(\widetilde{L})$. Then $f$ is a faithful monodromy-invariant function (see Definition 5.16).

The proposition is proven below in Lemmas 7.1 and 7.3. We provide an explicit and easily computable classification of the isometry classes of the pairs $(\widetilde{L}, e)$ in Lemma 6.5. 
Let $L_{0}$ be a rank 2 even lattice of signature $(1,1)$. Let $I_{n}\left(L_{0}\right) \subset L_{0}$ be the subset of primitive classes $e$ with $(e, e)=2-2 n$. Let $I_{L_{0}, n}(X) \subset I_{n}^{\prime \prime}(X)$ be the subset consisting of classes $e$, such that the lattice $\widetilde{L}$ in equation (6.1) is isometric to $L_{0}$. Consider the function

$$
f: I_{L_{0}, n}(X) \rightarrow I_{n}\left(L_{0}\right) / O\left(L_{0}\right),
$$

which sends the pair $(X, e)$ to the isometry class of the pair $(\widetilde{L}, e)$. The faithfulness statement in Proposition 6.3 follows from the following general statement.

\section{LEMMA 6.4}

The function $f$, given in (6.2), is a faithful monodromy invariant.

\section{Proof}

Let $e_{1}, e_{2}$ be two classes in $I_{L_{0}, n}(X)$. Denote by $\widetilde{L}_{j}$ the primitive rank 2 sublattice of $\widetilde{\Lambda}$ associated to $e_{j}$ in equation (6.1), via a primitive embedding $\iota: H^{2}(X, \mathbb{Z}) \rightarrow$ $\widetilde{\Lambda}$ in the canonical $O(\widetilde{\Lambda})$-orbit, $j=1,2$. Denote $\iota\left(e_{j}\right)$ by $e_{j}$ as well.

Assume that $f\left(e_{1}\right)=f\left(e_{2}\right)$. Then there exists an isometry $g: \widetilde{L}_{1} \rightarrow \widetilde{L}_{2}$, such that $g\left(e_{1}\right)=e_{2}$. Let $v \in \widetilde{\Lambda}$ be a generator of $\iota\left[H^{2}(X, \mathbb{Z})\right]^{\perp}$. Then $v$ is orthogonal to $e_{j}$. Hence, $g(v)=v$ or $g(v)=-v$. If $g(v)=-v$, set $g^{\prime}:=-\left(R_{e_{2}} \circ g\right)$. Then $g^{\prime}: \widetilde{L}_{1} \rightarrow \widetilde{L}_{2}$ is an isometry satisfying $g^{\prime}\left(e_{1}\right)=e_{2}$ and $g^{\prime}(v)=v$. Hence, we may assume that $g(v)=v$.

There exists an isometry $\gamma \in O_{+}(\widetilde{\Lambda})$, such that $\gamma\left(\widetilde{L}_{1}\right)=\widetilde{L}_{2}$ and $\gamma$ restricts to $\widetilde{L}_{1}$ as $g$, by [Ni, Theorem 1.14.4]. Then $\gamma(v)=v$, and so $\gamma \circ \iota=\iota \circ \mu$, for some isometry $\mu \in O_{+} H^{2}(X, \mathbb{Z})$. The fact that the isometry $\mu$ extends to $\widetilde{\Lambda}$ implies that $\mu$ belongs to $\operatorname{Mon}^{2}(X)$, by [Ma3, Theorem 1.6] (see also [Ma3, Lemma 4.10(3)]). Now $\iota\left(\mu\left(e_{1}\right)\right)=\gamma\left(\iota\left(e_{1}\right)\right)=\iota\left(e_{2}\right)$. So $\mu\left(e_{1}\right)=e_{2}$.

\subsection{Isometry orbits in three rank two lattices}

Set

$$
M_{U}:=\left(\begin{array}{cc}
0 & -1 \\
-1 & 0
\end{array}\right), \quad M_{H_{e v}}:=\left(\begin{array}{cc}
2 & 0 \\
0 & -2
\end{array}\right), \quad M_{U(2)}:=\left(\begin{array}{cc}
0 & -2 \\
-2 & 0
\end{array}\right) .
$$

Given an integer $m$, let $\mathcal{F}(m)$ be the set of unordered pairs $\{r, s\}$ of positive integers, such that $r s=m$ and $\operatorname{gcd}(r, s)=1$. Set

$$
\begin{aligned}
& \Sigma_{n}(U):=\mathcal{F}(n-1), \\
& \Sigma_{n}(U(2)):=\mathcal{F}([n-1] / 2), \quad \text { if } n \text { is odd, } \\
& \Sigma_{n}\left(H_{e v}\right):= \begin{cases}\mathcal{F}(n-1) & \text { if } n \neq \equiv 1 \text { (modulo) } 4, \\
\mathcal{F}([n-1] / 4) & \text { if } n \equiv 1 \text { (modulo) } 4 .\end{cases}
\end{aligned}
$$

Table 1 summarizes how the statements of Proposition 6.3 and Lemma 6.5 determine the lattice $\widetilde{L}$ and the pair $\{r, s\}$ in terms of $(e, e), \operatorname{div}(e, \bullet), n$, and $\{\rho, \sigma\}$. 
Table 1

\begin{tabular}{|c|c|c|c|c|c|c|c|}
\hline & $(e, e)$ & $\operatorname{div}(e, \bullet)$ & $n$ & $\rho \sigma$ & $\widetilde{L}$ & $\{r, s\}$ & $r \cdot s$ \\
\hline$(1)$ & $2-2 n$ & $2 n-2$ & $\geq 2$ & $4 n-4$ & $U$ & $\left\{\frac{\rho}{2}, \frac{\sigma}{2}\right\}$ & $n-1$ \\
\hline$(2)$ & $2-2 n$ & $n-1$ & even & $n-1$ & $H_{e v}$ & $\{\rho, \sigma\}$ & $n-1$ \\
\hline$(3)$ & $2-2 n$ & $n-1$ & odd & $2 n-2$ & $U(2)$ & $\left\{\frac{\rho}{2}, \frac{\sigma}{2}\right\}$ & $(n-1) / 2$ \\
\hline$(4)$ & $2-2 n$ & $n-1$ & $\equiv 1$ modulo 8 & $n-1$ & $H_{e v}$ & $\left\{\frac{\rho}{2}, \frac{\sigma}{2}\right\}$ & $(n-1) / 4$ \\
\hline
\end{tabular}

In line (3) cases where $n \equiv 1$ modulo 8 occur as well.

\section{LEMMA 6.5}

Let $\widetilde{L}$ be $U, H_{e v}$, or $U(2)$, and let $e \in I_{n}(\widetilde{L}), n \geq 2$. Choose a generator $v$ of the sublattice of $\widetilde{L}$ orthogonal to $e$.

(1) Let $\rho$ be the largest positive integer such that $(e+v) / \rho$ is an integral class of $\widetilde{L}$. Define the integer $\sigma$ similarly using $(e-v)$. Then $\operatorname{gcd}(\rho, \sigma)$ is 1 or 2 .

(2) The integers $r:=\rho / \operatorname{gcd}(\rho, \sigma)$ and $s:=\sigma / \operatorname{gcd}(\rho, \sigma)$ have the following properties.

(a) If $\widetilde{L}=U$, then $r s=n-1$, and the classes $\alpha:=(e+v) / 2 r$ and $\beta:=$ $(e-v) / 2 s$ form a basis of $\widetilde{L}$ with Gram matrix $M_{U}$.

(b) If $\widetilde{L}=U(2)$, then $n$ is odd, $r s=(n-1) / 2$, and the classes $\alpha:=(e+v) / 2 r$ and $\beta:=(e-v) / 2 s$ form a basis of $\widetilde{L}$ with Gram matrix $M_{U(2)}$.

(c) If $\widetilde{L}=H_{e v}$ and $n$ is even, then $r s=n-1$ and the classes

$$
\alpha:=\frac{1}{2}\left[\frac{e+v}{r}-\frac{e-v}{s}\right] \quad \text { and } \quad \beta:=\frac{1}{2}\left[\frac{e+v}{r}+\frac{e-v}{s}\right]
$$

form a basis of $\widetilde{L}$ with Gram matrix $M_{H_{e v}}$.

(d) If $\widetilde{L}=H_{e v}$ and $n$ is odd, then $n \equiv 1$ modulo 4 , rs $=(n-1) / 4$, and the classes

$$
\alpha:=\frac{1}{2}\left[\frac{e+v}{2 r}-\frac{e-v}{2 s}\right] \quad \text { and } \quad \beta:=\frac{1}{2}\left[\frac{e+v}{2 r}+\frac{e-v}{2 s}\right]
$$

form a basis of $\widetilde{L}$ with Gram matrix $M_{H_{e v}}$.

(3) If we replace $v$ by $-v$, then $(r, s)$ gets replaced by $(s, r)$.

(4) Let $r s: I_{n}(\widetilde{L}) \rightarrow \Sigma_{n}(\widetilde{L})$ be the function which assigns to a class $e \in I_{n}(\widetilde{L})$ the unordered pair $\{r, s\}$ occurring in the above factorization. Then rs factors through a one-to-one correspondence

$$
\overline{r s}: I_{n}(\widetilde{L}) / O(\widetilde{L}) \longrightarrow \Sigma_{n}(\widetilde{L}) .
$$

\section{Proof}

Let $\left\{u_{1}, u_{2}\right\}$ be a basis of $\widetilde{L}$ with Gram matrix $M_{\widetilde{L}}$. Observe first that $O(\widetilde{L})$ is isomorphic to $\mathbb{Z} / 2 \mathbb{Z} \times \mathbb{Z} / 2 \mathbb{Z}$. Indeed, each of $O(U)$ and $O(U(2))$ is generated by $-i d$ and the isometry, which interchanges $u_{1}$ and $u_{2} . O\left(H_{e v}\right)$ is generated by the two commuting reflections with respect to $u_{1}$ and $u_{2}$. Write

$$
e=a u_{1}+b u_{2} \text {. }
$$


Case $\widetilde{L}=U$. We have $n-1=-(e, e) / 2=a b$ and $\operatorname{gcd}(a, b)=1$, since $e$ is primitive. Note also that $a$ and $b$ have the same sign. Set $v:=a u_{1}-b u_{2}$. Then $(e+$ $v) / 2 a=u_{1}$ and $(e-v) / 2 b=u_{2}$. Thus $r=|a|$ and $s=|b|$, and part (2) holds. Part (3) is clear. Part (4) follows from part (3) and the identification of $O(U)$ above.

Case $\widetilde{L}=U(2)$. We may identify the free abelian groups underlying $U$ and $U(2)$, so that the bilinear form on $U(2)$ is 2 times that of $U$. The statement of the lemma follows immediately from the case $\widetilde{L}=U$.

Case $\widetilde{L}=H_{e v}$. We have $2-2 n=(e, e)=2(a-b)(a+b)$. So $b-a$ and $b+a$ have the same sign, since $n \geq 2$. If $n$ is odd, then both $a$ and $b$ are odd, since $\operatorname{gcd}(a, b)=1$ and $(a-b)(a+b)$ is even. If $n$ is even, then $\{a, b\}$ consists of one odd and one even integer. Furthermore,

$$
\operatorname{gcd}(b-a, b+a)=\operatorname{gcd}(b-a, 2 a)= \begin{cases}1 & \text { if } n \text { is even } \\ 2 & \text { if } n \text { is odd }\end{cases}
$$

Choose $v=b u_{1}+a u_{2}$. We have

$$
u_{1}=\frac{1}{2}\left[\frac{e+v}{a+b}-\frac{e-v}{b-a}\right], \quad u_{2}=\frac{1}{2}\left[\frac{e+v}{a+b}+\frac{e-v}{b-a}\right] .
$$

Hence, $r=|a+b|$ and $s=|b-a|$, if $n$ is even, and $r=|a+b| / 2$ and $s=|b-a| / 2$, if $n$ is odd. The rest is similar to the case $\widetilde{L}=U$.

\section{Monodromy invariants of monodromy-reflective classes}

Fix $n \geq 2$. Let $X$ be a (Kähler) irreducible holomorphic symplectic manifold of $K 3^{[n]}$-type. We define in this section the monodromy invariant function $r s$ of Proposition 1.8 and prove that proposition. Part (2) of the proposition was treated in [Ma3, Lemma 8.9]. We thus consider only part (1). We will relate this latter part to Proposition 6.3 and prove Proposition 6.3.

It will be convenient to use the following normalization. Fix an isometry $\widetilde{\Lambda} \cong K(S)$, for some $K 3$ surface $S$, and use Mukai's notation for classes in the Mukai lattice $K(S)$. The isometry group $O(\widetilde{\Lambda})$ acts transitively on the set of primitive classes in $\widetilde{\Lambda}$ of degree $2 n-2$. Hence, we may choose the embedding $\iota: H^{2}(X, \mathbb{Z}) \rightarrow \widetilde{\Lambda}$, so that $v=(1,0,1-n)$ is orthogonal to the image of $\iota$. Then $v^{\perp}=H^{2}(S, \mathbb{Z}) \oplus \mathbb{Z} \delta$, where $\delta:=(1,0, n-1)$. Thus

$$
e=x+t \delta \text {, }
$$

for some integer $t$ and a class $x \in H^{2}(S, \mathbb{Z})$.

\subsection{The divisibility case $\operatorname{div}(e, \bullet)=(e, e)$}

Let $I_{n}(X) \subset H^{2}(X, \mathbb{Z})$ be the subset of all primitive classes $e$, satisfying $(e, e)=$ $2-2 n$ and $\operatorname{div}(e, \bullet)=2 n-2$. Recall that $\Sigma_{n}(U)$ is the set of unordered pairs $\{r, s\}$ of positive integers, such that $r s=n-1$ and $\operatorname{gcd}(r, s)=1$.

\section{LEMMA 7.1}

If e belongs to $I_{n}(X)$, then $\widetilde{L}$ is isometric to the unimodular hyperbolic plane $U$. 
Denote by

$$
r s: I_{n}(X) \longrightarrow \Sigma_{n}(U)
$$

the composition of the function $f: I_{n}(X) \rightarrow I_{n}(U) / O(U)$, defined in equation (6.2), with the bijection $\overline{r s}: I_{n}(U) / O(U) \rightarrow \Sigma_{n}(U)$ constructed in Lemma 6.5. Then the function $r s: I_{n}(X) \rightarrow \Sigma_{n}(U)$ is surjective and a faithful monodromy invariant (see Definition 5.16).

Proof

Write $e=x+t \delta$ as in equation (7.1). The assumption that $\operatorname{div}(e, \bullet)=2 n-2$ implies that $x=(2 n-2) \xi$, for a class $\xi \in H^{2}(S, \mathbb{Z})$. We clearly have the equality

$$
2-2 n=(e, e)=(x, x)+t^{2}(\delta, \delta)=(2-2 n)^{2}(\xi, \xi)+(2-2 n) t^{2} .
$$

Hence, we get the equality

$$
t^{2}-1=(2 n-2)(\xi, \xi)
$$

Consequently, $4 n-4$ divides $(t-1)(t+1)$. Thus $n-1$ divides $(t-1)(t+1) / 4$. Now $\operatorname{gcd}((t-1) / 2,(t+1) / 2)=1$. We get a unique factorization $n-1=r s$, where $s$ divides $(t-1) / 2, r$ divides $(t+1) / 2$, and $\operatorname{gcd}(r, s)=1$. We may assume that $s$ is odd, possibly after replacing the embedding $\iota$ by $-\iota$, which replaces $t$ by $-t$.

Using the above factorization $n-1=r s$, we get

$$
\begin{aligned}
e+v & =2 r \alpha, \quad \text { where } \alpha:=\left(\frac{t+1}{2 r}, s \xi, \frac{(t-1) s}{2}\right), \\
e-v & =2 s \beta, \quad \text { where } \beta:=\left(\frac{t-1}{2 s}, r \xi, \frac{(t+1) r}{2}\right)
\end{aligned}
$$

and the classes $\alpha$ and $\beta$ belongs to $\widetilde{L}$. The Gram matrix of $\{\alpha, \beta\}$ is

$$
\left(\begin{array}{ll}
(\alpha, \alpha) & (\alpha, \beta) \\
(\alpha, \beta) & (\beta, \beta)
\end{array}\right)=\left(\begin{array}{ll}
(e+v, e+v) / 4 r^{2} & (e+v, e-v) / 4 r s \\
(e+v, e-v) / 4 r s & (e-v, e-v) / 4 s^{2}
\end{array}\right)=\left(\begin{array}{cc}
0 & -1 \\
-1 & 0
\end{array}\right) .
$$

We conclude that $\operatorname{span}\{\alpha, \beta\}$ is a unimodular sublattice of $\widetilde{\Lambda}$. Hence, $\widetilde{L}=$ $\operatorname{span}\{\alpha, \beta\}$ and $\widetilde{L} \cong U$.

The function $r s$ is shown to be surjective in Example 7.2. The faithfulness of the monodromy invariant $r s$ was proven in Lemma 6.4 .

\section{EXAMPLE 7.2}

Compare this with Section 2.2 above. Choose a factorization $n-1=r s$, with $s \geq$ $r>0$, and $\operatorname{gcd}(r, s)=1$. Let $S$ be a projective $K 3$ surface, $v=(r, 0,-s) \in K(S)$, $H$ a $v$-generic polarization, and $X=M_{H}(r, 0,-s)$. Let $\iota: H^{2}\left(M_{H}(r, 0,-s), \mathbb{Z}\right) \hookrightarrow$ $K(S)$ be the embedding given in (2.3). Set $e:=\theta(r, 0, s)$, where $\theta$ is Mukai's isometry given in equation (2.2). The class $e$ is monodromy reflective and $\operatorname{div}(e, \bullet)=$ $2 n-2$. Now $(v+e) / 2 r=(1,0,0),(e-v) / 2 s=(0,0,1)$, and $\widetilde{L} \cong U$. We get $r s(e)=\{r, s\}$, by Lemma 6.5 . 
7.2. The divisibility case $\operatorname{div}(e, \bullet)=(e, e) / 2$

Let $n$ be an integer $\geq 2$. Let $I_{n}^{\prime}(X) \subset H^{2}(X, \mathbb{Z})$ be the subset of all primitive classes $e$ satisfying $(e, e)=2-2 n$, and $\operatorname{div}(e, \bullet)=n-1$. Set

$$
\Sigma_{n}^{\prime}:= \begin{cases}\Sigma_{n}\left(H_{e v}\right) & \text { if } n \text { is even, } \\ \Sigma_{n}(U(2)) & \text { if } n \text { is odd but } n \neq 1 \text { modulo } 8 \\ \Sigma_{n}(U(2)) \cup \Sigma_{n}\left(H_{e v}\right) & \text { if } n \equiv 1 \text { modulo } 8 .\end{cases}
$$

In each of the above three cases, let $\mathcal{I C}_{n}$ be the union of the sets $I_{n}(\widetilde{L}) / O(\widetilde{L})$ as $\widetilde{L}$ ranges through the one or two lattices appearing.

\section{LEMMA 7.3}

Let e be a class in $I_{n}^{\prime}(X)$.

(1) If $n$ is even, then $\widetilde{L}$ is isometric to $H_{e v}$.

(2) If $n$ is odd, then $\widetilde{L}$ is isometric to $U(2)$ or to $H_{e v}$. The latter occurs only if $n \equiv 1$ modulo 8 and $\rho \sigma=n-1$.

In both cases, let

$$
r s: I_{n}^{\prime}(X) \longrightarrow \Sigma_{n}^{\prime}
$$

be the composition of the function $f: I_{n}^{\prime}(X) \rightarrow \mathcal{I C}_{n}$, defined in equation (6.2), with the injection $\overline{r s}: \mathcal{I C}_{n} \rightarrow \Sigma_{n}^{\prime}$, constructed in Lemma 6.5. Then the function rs $: I_{n}^{\prime}(X) \rightarrow \Sigma_{n}^{\prime}$ is surjective and a faithful monodromy invariant (see Definition 5.16).

Proof

Let us first observe that $\widetilde{L}$ cannot be unimodular. Assume otherwise. Then $\widetilde{\Lambda}$ decomposes as an orthogonal direct sum $\widetilde{L} \oplus \widetilde{L}^{\perp}$. Consequently, $v^{\perp}$ decomposes as the orthogonal direct sum $\widetilde{L}^{\perp} \oplus \mathbb{Z}\{e\}$. But then $\operatorname{div}(e, \bullet)=2 n-2$.

We keep the normalization $e=x+t \delta$ of equation (7.1). The assumption that $\operatorname{div}(e, \bullet)=n-1$ implies that $x=(n-1) \xi$, for a class $\xi \in H^{2}(X, \mathbb{Z})$. We have the equality

$$
\left(t^{2}-1\right)=\frac{(n-1)(\xi, \xi)}{2} .
$$

Hence, $n-1$ divides $t^{2}-1$.

Case $n$ is even. Then $n-1$ is odd. Set

$$
r:=\operatorname{gcd}(t+1, n-1), \quad s:=\operatorname{gcd}(t-1, n-1) .
$$

Then both $r$ and $s$ are odd and $\operatorname{gcd}(r, s)$ divides $\operatorname{gcd}(t-1, t+1)$. We conclude that $\operatorname{gcd}(r, s)=1$ and $r s$ divides $n-1$. Now $n-1$ divides $(t-1)(t+1)$. Thus, $n-1$ divides $r s$, and so $r s=n-1$. Set

$$
\begin{aligned}
& \alpha:=\frac{1}{2}\left[\frac{e+v}{r}-\frac{e-v}{s}\right]=\frac{1}{2}\left(\frac{t+1}{r}-\frac{t-1}{s},(s-r) \xi,(s-r) t-s-r\right), \\
& \beta:=\frac{1}{2}\left[\frac{e+v}{r}+\frac{e-v}{s}\right]=\frac{1}{2}\left(\frac{t+1}{r}+\frac{t-1}{s},(s+r) \xi,(s+r) t+r-s\right) .
\end{aligned}
$$


Note the equality $(t+1) / r-(t-1) / s=((s-r) t+s+r) / r s$ and the fact that the denominator is odd, while the numerator is even. Hence, $\alpha, \beta$ are integral classes of $\widetilde{\Lambda}$, and $\left(\begin{array}{cc}(\alpha, \alpha) & (\alpha, \beta) \\ (\alpha, \beta) & (\beta, \beta)\end{array}\right)=\left(\begin{array}{cc}2 & 0 \\ 0 & -2\end{array}\right)$.

\section{CLAIM 7.4}

$\widetilde{L}=\operatorname{span}\{\alpha, \beta\}$.

Proof

Suppose otherwise. Then $\widetilde{L}$ strictly contains $L^{\prime}:=\operatorname{span}\{\alpha, \beta\}$. Let $d$ be the index of $L^{\prime}$ in $\widetilde{L}$. Then the determinant of the Gram matrix of $\widetilde{L}$ is $d^{2}$ times the determinant of the Gram matrix of $L^{\prime}$. The latter determinant is -4 . It follows that $\widetilde{L}$ is unimodular, a contradiction.

Case $n$ is odd. Then $t$ is odd, by equation (7.2). Set

$$
r:=\operatorname{gcd}\left(\frac{n-1}{2}, \frac{t+1}{2}\right), \quad s:=\operatorname{gcd}\left(\frac{n-1}{2}, \frac{t-1}{2}\right) .
$$

Then $r s$ divides $(n-1) / 2$, since $\operatorname{gcd}((t+1) / 2,(t-1) / 2)=1$.

Case $n$ is odd and $(\xi, \xi) / 2$ is even. Then $(n-1) / 2$ divides $(t+1)(t-1) / 4$, by equation (7.2). Hence, $r s=(n-1) / 2$. Set

$$
\begin{aligned}
\alpha & :=\frac{e+v}{2 r}=\left(\frac{t+1}{2 r}, s \xi, s(t-1)\right), \\
\beta & :=\frac{e-v}{2 s}=\left(\frac{t-1}{2 s}, r \xi, r(t+1)\right) .
\end{aligned}
$$

Then $\alpha$ and $\beta$ are integral classes of $\widetilde{\Lambda}$, and $\left(\begin{array}{cc}(\alpha, \alpha) & (\alpha, \beta) \\ (\alpha, \beta) & (\beta, \beta)\end{array}\right)=\left(\begin{array}{cc}0 & -2 \\ -2 & 0\end{array}\right)$. We conclude the equality $\widetilde{L}=\operatorname{span}\{\alpha, \beta\}$, by the argument used in Claim 7.4.

Case $n$ is odd and $(\xi, \xi) / 2$ is odd. Let $2^{k}$ be the largest power of 2 which divides $t^{2}-1$. Then $k \geq 3$. Furthermore, $2^{k}$ is also the largest power of 2 which divides $n-1$, by equation (7.2). Thus $n \equiv 1$ (modulo 8 ). The set $\{r, s\}$ consists of one odd and one even integer. Say $s$ is odd. Then $2^{k-2}$ is the largest power of 2 , which divides $r$. We conclude that $r s=(n-1) / 4$. Furthermore, both $(t+1) / 2 r$ and $(t-1) / 2 s$ are odd. Set

$$
\begin{aligned}
\alpha & :=\frac{1}{2}\left[\frac{e+v}{2 r}+\frac{e-v}{2 s}\right]=\frac{1}{2}\left(\frac{t+1}{2 r}+\frac{t-1}{2 s},(2 s+2 r) \xi, 2 s(t-1)+2 r(t+1)\right), \\
\beta & :=\frac{1}{2}\left[\frac{e+v}{2 r}-\frac{e-v}{2 s}\right]=\frac{1}{2}\left(\frac{t+1}{2 r}-\frac{t-1}{2 s},(2 s-2 r) \xi, 2 s(t-1)-2 r(t+1)\right) .
\end{aligned}
$$

Then $\alpha$ and $\beta$ are integral classes of $\widetilde{\Lambda}$ and $\left(\begin{array}{cc}(\alpha, \alpha) & (\alpha, \beta) \\ (\alpha, \beta) & (\beta, \beta)\end{array}\right)=\left(\begin{array}{cc}-2 & 0 \\ 0 & 2\end{array}\right)$. We conclude the equality $\widetilde{L}=\operatorname{span}\{\alpha, \beta\}$, by the argument used in Claim 7.4.

The function $r s$ is shown to be surjective in Examples 7.5 and 7.6. The faithfulness of the monodromy invariant $r s$ was proven in Lemma 6.4.

EXAMPLE 7.5

Let $s>r \geq 1$ be positive integers with $\operatorname{gcd}(r, s)=1$. Set $n:=r s+1$. Let $S$ be a 
projective $K 3$ surface, set $v:=(r, 0,-s)$, and let $H$ be a $v$-generic polarization of $S$. Set $M:=M_{H}(v)$. Let $A$ be a primitive isotropic class in $H^{2}(S, \mathbb{Z})$. Set $e:=\theta(r,(n-1) A, s)$. Then $e$ is monodromy reflective and $\operatorname{div}(e, \bullet)=n-1$. If $n$ is even, then $(M, e)$ is an example of case 1 of Lemma 7.3, with $r s(e)=\{r, s\}$. If $n$ is odd, then $n-1=r s$ is even and precisely one of $r$ or $s$ is even. If $r$ is even, then $\rho=r$ and $\sigma=2 s$. If $s$ is even, then $\rho=2 r$ and $\sigma=s .(M, e)$ is an example of Lemma 7.3(2), with $\widetilde{L} \cong U(2)$ and $r s(e)=\{r / 2, s\}$, if $r$ is even, and $r s(e)=\{r, s / 2\}$, if $s$ is even.

\section{EXAMPLE 7.6}

We exhibit the next examples of the case of Lemma 7.3, where $X=S^{[n]}, n \equiv 1$ modulo 8 , and $\widetilde{L} \cong H_{e v}$. Set $n=8 k+1, k$ an integer $\geq 1$. Choose a factorization $2 k=r s$, with $r$ even, $s$ odd, and $\operatorname{gcd}(r, s)=1$. There exists an integer $\lambda$, such that $\lambda r \equiv-1$ modulo $s$, since $\operatorname{gcd}(s, r)=1$. If $\lambda$ is a solution, so is $\lambda+s$. Hence, we may assume that $\lambda$ is an odd and positive integral solution. Set $g:=[r \lambda+1] / s$. Then $g$ is a positive odd integer.

Let $S$ be a $K 3$ surface with a primitive class $\xi \in \operatorname{Pic}(S)$ of degree $(\xi, \xi)=2 \lambda g$. Set

$$
v:=(1,0,1-n) \quad \text { and } \quad e:=(2 \lambda r+1,(n-1) \xi,[2 \lambda r+1](n-1)) .
$$

Then $(e, e)=2-2 n$, by the two equalities

$$
(e, e)=(n-1)\left[2 \lambda g(n-1)-2\left(4\left\{r^{2} \lambda^{2}+r \lambda\right\}+1\right)\right]
$$

and $2 \lambda g(n-1)=8 r \lambda s g=8 r \lambda(r \lambda+1)$. The class $e$ is primitive, since

$$
\operatorname{gcd}(2 \lambda r+1, n-1)=\operatorname{gcd}(2 \lambda r+1,4 r s)=\operatorname{gcd}(2 \lambda r+1, s)=\operatorname{gcd}(-1, s)=1 .
$$

The classes $(e+v) / 2 s=\left(g, 2 r \xi, 4 \lambda r^{2}\right)$ and $(e-v) / 2 r=\left(\lambda, 2 s \xi, 4 g s^{2}\right)$ are integral and primitive. We conclude that $\widetilde{L} \cong H_{e v}$, by Proposition $6.3(1)$, and $r s(e)=$ $\{r, s\}$, by Lemma 6.5 .

\section{Numerical characterization of exceptional classes via Torelli}

Table 2 points to an example provided in this paper, for each possible value of the quadruple $\{n,(e, e), \operatorname{div}(e, \bullet), r s(e)\}$, for a monodromy reflective class $e$.

The congruence constraints on $n$ are necessary. If $(e, e)=-2$ and $\operatorname{div}(e, \bullet)=$ 2 , then $n \equiv 2$ (modulo 4), by [Ma3, Lemma 8.9]. If $r s(e)=\{2,(n-1) / 2\}$, then $n \equiv 3$ (modulo 4 ) in order for $\{2,(n-1) / 2\}$ to be a pair of relatively prime integers. If $(e, e)=2-2 n, \operatorname{div}(e, \bullet)=n-1, n>2$, and $r s(e)=\{1, n-1\}$, then $n$ must be even, since for odd $n$ the product of $r$ and $s$ is equal to $(n-1) / 2$ or $(n-1) / 4$, by Lemmas 7.3 and 6.5. This explains also the value of $n$ in the last three rows.

Proof of Theorem 1.11

Set $e:=c_{1}(L)$. The pair $(X, e)$ is deformation equivalent, in the sense of Definition 5.6 , to a pair $(M, c)$ appearing in Table 2, by Proposition 1.8 and Lem- 
Table 2

\begin{tabular}{|c|c|c|c|c|c|}
\hline$(e, e)$ & $\operatorname{div}(e, \bullet)$ & $\widetilde{L}$ & $\{r, s\}$ & $n$ & Reference \\
\hline-2 & 1 & $\mathrm{NA}$ & NA & $\geq 2$ & Examples $10.2,10.5$ \\
\hline-2 & 2 & NA & NA & $\begin{array}{c}\geq 6 \text { and } \\
\equiv 2 \bmod 4\end{array}$ & Example 10.6 \\
\hline-2 & 2 & $\mathrm{NA}$ & $\mathrm{NA}$ & $=2$ & Example 10.13 \\
\hline $2-2 n$ & $2 n-2$ & $U$ & $\{1, n-1\}$ & $\geq 2$ & Example 10.13 \\
\hline $2-2 n$ & $2 n-2$ & $U$ & $\{2,(n-1) / 2\}$ & $\begin{array}{c}\geq 7 \text { and } \\
\equiv 3 \bmod 4\end{array}$ & Lemma 10.16(2) \\
\hline $2-2 n$ & $2 n-2$ & $U$ & $\begin{array}{c}s>r>2 \\
\operatorname{gcd}(r, s)=1\end{array}$ & $=r s+1$ & Proposition 11.1 \\
\hline $2-2 n$ & $n-1$ & $H_{e v}$ & $\{1, n-1\}$ & $\geq 4$, even & Lemma 10.16(1) \\
\hline $2-2 n$ & $n-1$ & $U(2)$ & $\{1,(n-1) / 2\}$ & $\geq 3$, odd & Lemma 10.16(1) \\
\hline $2-2 n$ & $n-1$ & $H_{e v}$ & $\begin{array}{l}r \geq 3, s \geq 3 \\
\operatorname{gcd}(r, s)=1\end{array}$ & $\begin{array}{c}=r s+1 \\
\text { even }\end{array}$ & Lemma 11.2 \\
\hline $2-2 n$ & $n-1$ & $U(2)$ & $\begin{array}{l}r \geq 3, s \geq 2 \\
\operatorname{gcd}(r, s)=1\end{array}$ & $=2 r s+1$ & Lemma 11.2 \\
\hline $2-2 n$ & $n-1$ & $H_{e v}$ & $\begin{array}{c}r \text { even, } s \text { odd } \\
\operatorname{gcd}(r, s)=1\end{array}$ & $=4 r s+1$ & Example 11.3 \\
\hline
\end{tabular}

ma 5.17(1). $M$ is projective, and Theorem 1.11 holds for $(M, c)$, by the example referred to in Table 2. Suppose that $L$ is numerically effective. Then Theorem 1.11(1) follows for $(X, L)$, by Proposition 5.14.

Suppose next that $L$ is not numerically effective. We prove Theorem 1.11(2) by contradiction. Assume that part (2) fails. Then there exists a nonzero integer $k$, such that $h^{0}\left(X_{t}, L_{t}^{k}\right)>0$, for all $t \in D_{e}$. We may assume that the absolute value $|k|$ is minimal with the above property. Now $\operatorname{Pic}\left(X_{t}\right)$ is cyclic, for a generic $t \in D_{e}$. Hence, the linear system $\left|L_{t}^{k}\right|$ must have a member $E_{t}$, which is a prime divisor, by the minimality of $|k|$. It follows that $E_{t}$ is the unique member of the linear system, by [Bou, Proposition 3.13]. Hence, $h^{0}\left(X_{t}, L_{t}^{k}\right)=1$, away from a closed analytic proper subset $Z \subset D_{e}$.

Set $U:=D_{e} \backslash Z$, and let $\mathcal{X}_{U}$ be the restriction of the semiuniversal family from $\operatorname{Def}(X)$ to $U$. There exists an irreducible divisor $\mathcal{E} \subset \mathcal{X}_{U}$, which does not contain the fiber $X_{t}$, for any $t \in U$, and which intersects $X_{t}$ along a divisor in $\left|L_{t}^{k}\right|$, by the argument used in the proof of Proposition 5.2. The argument furthermore shows that there exists a closed analytic proper subset $Z_{1} \subset U$, such that the fiber $E_{t}$ of $\mathcal{E}$ is a prime divisor, over all points $t \in U \backslash Z_{1}$. We do not need the projectivity assumption, as it was used in the proof of Proposition 5.2 only to establish that the generic dimension of $h^{0}\left(X_{t}, L_{t}\right)$ is 1 , a fact which was already established above.

We conclude the existence of a pair $\left(X_{1}, e_{1}\right)$, parameterized by a point in $U \backslash Z_{1}$, such that $X_{1}$ is projective, by [Hu2, Proposition 21]. Let $L_{2}$ be the 
line bundle on $M$ with $c_{1}\left(L_{2}\right)=c$. Then $H^{0}\left(M, L_{2}^{d}\right)$ vanishes, for all nonzero integers $d$, since $L_{2}$ is not numerically exceptional, and the examples mentioned in Table 2 have this property, whenever $c$ is not numerically exceptional. Hence, $\left(X_{1}, e_{1}\right)$ and $(M, c)$ are not deformation equivalent, by Corollary 5.15. On the other hand, $\left(X_{1}, e_{1}\right)$ is deformation equivalent to $(X, e)$, and hence to $(M, c)$, a contradiction.

\section{Conditions for the existence of slope-stable vector bundles}

Let $S$ be a projective $K 3$ surface with a cyclic Picard group generated by an ample line bundle $H$. We assemble in Section 9.1 necessary conditions for the existence of locally free $H$-slope-stable sheaves (see Lemmas 9.2-9.4).

In Section 9.2 we bound the dimension of the locus Exc of $H$-stable sheaves, which are not locally free or not $H$-slope-stable. The sheaves $F$ considered all have the following involutive property: there exists an integer $t$, such that the classes in $K(S)$ of $F$ and $F^{*} \otimes H^{t}$ are equal. Equivalently, $c_{1}(F)=(t \cdot \operatorname{rank}(F) / 2) h$, for some integer $t$, where $h:=c_{1}(H)$. I thank Kota Yoshioka for pointing out that much of the content of Section 9.2 is essentially proven in Sections 2 and 3 of his paper [Y3]. Section 9.2 was not replaced by a citation, since the precise statements we need are not easily recovered from those of Yoshioka, as he was mainly concerned with proving that the locus Exc has codimension $\geq 1$, while we need that Exc have codimension $\geq 2$ in the subset of cases considered.*

The results of this section are only lightly used in Section 10 but are essential to the examples in Section 11.

\subsection{Necessary conditions}

Set $h:=c_{1}(H) \in H^{2}(S, \mathbb{Z})$ and $d:=\operatorname{deg}(H) / 2$.

\section{LEMMA 9.1}

Let $F$ be a locally free $H$-stable sheaf of rank $r$ satisfying $c_{1}(F)=(t r / 2) h$, for some integer $t$. Then $F^{*}$ is $H$-stable, if and only if $F$ is $H$-slope-stable. In particular, if $r=2$, then $F$ is $H$-slope-stable.

Proof

After tensorization by a power of $H$, we may reduce to the case where either $c_{1}(F)=0$, or $r=2 \rho$ is even and $c_{1}(F)=\rho h$. Assume that we are in one of these cases. If $c_{1}(F)=0$, set $L:=\mathcal{O}_{S}$. If $c_{1}(F)=\rho h$, set $L:=H$. In either case, we have the equality $[F]=\left[F^{*} \otimes L\right]$ of classes in $K(S)$. Furthermore, a sheaf $G$ is $H$-stable if and only if $G \otimes L$ is $H$-stable.

If $F$ is $H$-slope-stable, then so is $F^{*}$. Hence $F^{*}$ is $H$-stable as well. $F$ is $H$-slope-semistable, since it is $H$-stable. Suppose that $F$ is not $H$-slope-stable. Then there exists a saturated subsheaf $F_{1} \subset F$, of rank (say) $r_{1}$, with $c_{1}\left(F_{1}\right)=$

\footnotetext{
${ }^{*}$ For some cases Yoshioka does state that the codimension is $\geq 2$ (see [Y3, Lemma 3.1]) but under an assumption that excludes some cases which we need.
} 
$\left(r_{1} / r\right) c_{1}(F)$ and $0<r_{1}<r$. Set $F_{2}:=F / F_{1}$ and $r_{2}:=r-r_{1} . H$-stability of $F$ yields the inequality $\chi\left(F_{2}\right) / r_{2}>\chi(F) / r$. We get the injective homomorphism $F_{2}^{*} \rightarrow F^{*}$ and the inequalities

$$
\frac{\chi\left(F_{2}^{*} \otimes L\right)}{r_{2}}=\frac{\chi\left(F_{2}^{* *}\right)}{r_{2}} \geq \frac{\chi\left(F_{2}\right)}{r_{2}}>\frac{\chi(F)}{r}=\frac{\chi\left(F^{*} \otimes L\right)}{r} .
$$

Hence, $F^{*} \otimes L$ is $H$-unstable. Consequently, $F^{*}$ is $H$-unstable.

If $r=2$, then $F^{*} \otimes L$ is isomorphic to $F$ and thus $F^{*}$ is $H$-stable.

\section{LEMMA 9.2}

Let $F$ be a locally free $H$-slope-stable sheaf of class $v=(r, 0,-s)$. Then $v=$ $(1,0,1)$ or $s \geq r \geq 2$.

\section{Proof}

If $\operatorname{rank}(F)=1$, then $F$ is isomorphic to $\mathcal{O}_{S}$, since $F$ is locally free, and so $s=$ -1 . Assume that $v \neq(1,0,1)$. Then $H^{0}(F)$ vanishes, by the $H$-stability of $F$. Similarly, $H^{2}(F)^{*} \cong H^{0}\left(F^{*}\right)=(0)$, by the $H$-slope-stability of $F^{*}$. Thus, $r-s=$ $\chi(F)=-\operatorname{dim} H^{1}(F) \leq 0$.

Lemma 9.2 states a necessary cohomological condition for the existence of a locally free $H$-slope-stable sheaf of class $v$ with $c_{1}(v)=0$ (slope 0 ). The condition states that $\chi(v) \leq 0$, unless $v$ is the class $u=(1,0,1)$ of the trivial line bundle. If $\chi(v) \leq 0$, then the locus of sheaves with nonzero global sections is expected to have positive codimension. The condition $\chi(v) \leq 0$ translates to $(u, v) \geq 0$. The following lemma states a similar cohomological condition, for a class $v$ with a nonzero slope. The role of the trivial line bundle is replaced next by a simple and rigid sheaf $E$ of the same slope as $v$.

\section{LEMMA 9.3}

Let $F$ be a locally free $H$-slope-stable sheaf of class $v=(2 r, r h,-b)$, where $r>0$, $\operatorname{gcd}(r, b)=1,(h, h)=2 d$, and $d$ is an odd integer. Set $u:=(2, h,(d+1) / 2)$. If $(v, v)=-2$, then $v=u$. Otherwise, $(v, u) \geq 0$ and $(v, u)$ is even. Furthermore, $(v, u)=0$, if and only if $v=(2, h,(d-1) / 2)$.

\section{Proof}

$M_{H}(u)$ consists of a single isomorphism class. Let $E$ be an $H$-stable sheaf of class $u$. Then $E$ is necessarily $H$-slope-stable and locally free (see $[\mathrm{Mu}]) . M_{H}(v)$ is nonempty, by assumption. Let $2 n$ be its dimension. Then $2 n-2=(v, v)=$ $2 d r^{2}+4 r b$, and $b=\left(n-1-d r^{2}\right) / 2 r$. If $n=0$, then $r=1$, since $r$ divides $(v, v) / 2$. We conclude that $v=u$, if $(v, v)=-2$.

Assume that $v \neq u$. Then $n \geq 1$. We get the inequality

$$
\frac{\chi(u)}{2}=\frac{5+d}{4}>\frac{(4+d) r^{2}+1-n}{4 r^{2}}=\frac{\chi(v)}{2 r} .
$$


Thus, $\operatorname{Hom}(E, F)=0$. Similarly,

$$
\operatorname{Ext}^{2}(E, F)^{*} \cong \operatorname{Hom}(F, E) \cong \operatorname{Hom}\left(E^{*}, F^{*}\right) \cong \operatorname{Hom}\left(E^{*} \otimes H, F^{*} \otimes H\right)=0,
$$

since $E^{*} \otimes H \cong E$ and $F^{*} \otimes H$ is an $H$-slope-stable sheaf of class $v$. Thus, $(v, u)=$ $-\chi(v, u) \geq 0$. Furthermore, $(v, u)=2[r(d-1) / 2+b]$. If $(v, u)=0$, then $r$ divides $b$. Hence, $r=1$, since $\operatorname{gcd}(r, b)=1$. If $r=1$ and $(v, u)=0$, then $b=(1-d) / 2$, as claimed.

\section{LEMMA 9.4}

Let $F$ be a locally free $H$-slope-stable sheaf of class $v=(2 r, r h,-b)$, where $r>0$, $\operatorname{gcd}(r, b)=1,(h, h)=2 d$, and $d$ is an even integer. Set $u:=(2, h, d / 2)$. If $(v, v)=$ 0 , then $v=u$. Otherwise, $(u, v)$ is a positive even number.

This lemma has a cohomological interpretation as well. $M_{H}(u)$ is twodimensional, and it parameterizes locally free $H$-slope-stable sheaves (see $[\mathrm{Mu}]$ ). Let $B \subset M_{H}(u) \times M_{H}(v)$ be the correspondence consisting of pairs $(E, F)$, with nonvanishing $\operatorname{Hom}(E, F)$. The lemma states that if $v \neq u$, then the expected codimension $(u, v)+1$ of $B$ is larger than 2 , and so $B$ is not expected to surject onto $M_{H}(v)$.

\section{Proof}

Set $n:=(1 / 2) \operatorname{dim}_{\mathbb{C}} M_{H}(v)=1+(v, v) / 2$. If $n=1$, then $(v, v)=0$, and so $v=$ $k u$, for some positive integer $k$. If $k>1$, then the moduli space $M_{H}(k u)$ is the $k$ th symmetric product of $M_{H}(u)$ and it consists entirely of $H$-unstable but $H$ semistable sheaves. We are assuming however the existence of an $H$-slope-stable sheaf $F$ of class $v$. Hence, $k=1$ and $v=u$.

Assume that $n>1$. We get

$$
\frac{\chi(v)}{2 r}=\frac{(4+d) r^{2}-n+1}{4 r^{2}}<\frac{4+d}{4}=\frac{\chi(u)}{2} .
$$

The normalized Hilbert polynomial $p$ of a sheaf $G$ of positive rank is the Hilbert polynomial divided by the $\operatorname{rank} p(n):=\chi\left(G \otimes H^{n}\right) / \operatorname{rank}(G)$. The first two leading terms in the normalized Hilbert polynomials of $u$ and $v$ are equal, and the constant terms are related by the above inequality. $\operatorname{Hence} \operatorname{Hom}(E, F)=0$, for every $H$-slope-stable sheaf $E$ of class $u$. Such a sheaf $E$ is necessarily locally free, and so $E^{*} \otimes H$ is $H$-slope-stable of class $u$. We get also the vanishing of $\operatorname{Ext}^{2}(E, F)$, by the argument used in the proof of Lemma 9.3. We conclude the inequality $(u, v)=-\chi\left(E^{*} \otimes F\right) \geq 0$. Furthermore, $(u, v)=d r+2 b$ is even. If $(u, v)=0$, then $(v, v)=0$, and so $v=u$. The lemma follows.

\subsection{Sufficient conditions}

9.2.1. The case $c_{1}(v)=0$

Let $r, s$ be integers satisfying $s>r>2$ and $\operatorname{gcd}(r, s)=1$. Set $n:=r s+1$ and $v:=(r, 0,-s)$. A sheaf $F$ of class $v$ is $H$-stable if and only if it is $H$-semistable. Hence, $M_{H}(v)$ is smooth and projective of dimension $2 n$. Let Exc $\subset M$ be the 
locus of $H$-stable sheaves of class $v$ that are not locally free or not $H$-slope-stable. Exc is clearly a closed subset of $M_{H}(v)$.

\section{LEMMA 9.5}

Exc has codimension at least 2 in $M$.

\section{Proof}

We will use the following notation, in order for large parts of the proof to generalize to a proof of Lemma 9.6. Let $u:=(1,0,1)$ be the class of $\mathcal{O}_{S}$. Set $\epsilon:=\operatorname{rank}(u)=1$.

Step 1: Jun Li's morphism to the Uhlenbeck-Yau compactification. Let $Y_{H}(w)$ be the moduli space of $H$-slope-stable locally free sheaves of class $w \in K(S)$. Let $v_{1}, \ldots, v_{k}$ be distinct classes in $K(S)$, with $v_{i}=\left(r_{i}, 0,-s_{i}\right), r_{i}>0,\left(v_{i}, v_{i}\right) \geq-2$. Let $d_{1}, \ldots, d_{k}$ be positive integers satisfying

$$
r=\sum_{i=1}^{k} d_{i} r_{i} \quad \text { and } \quad t(\vec{v}, \vec{d}):=s-\left(\sum_{i=1}^{k} d_{i} s_{i}\right) \geq 0 .
$$

Denote the $d$ th symmetric product of $Y_{H}\left(v_{i}\right)$ by $Y_{H}\left(v_{i}\right)^{(d)}$. Set

$$
Y(\vec{v}, \vec{d}):=\prod_{i=1}^{k} Y_{H}\left(v_{i}\right)^{\left(d_{i}\right)} \times S^{(t(\vec{v}, \vec{d}))} .
$$

Note that for $Y_{H}\left(v_{i}\right)$ to be nonempty, $v_{i}=\left(r_{i}, 0,-s_{i}\right)$ should satisfy

$$
r_{i}=-s_{i}=1 \quad \text { or } \quad s_{i} \geq r_{i} \geq 2,
$$

by Lemma 9.2 . If $r_{i}=-s_{i}=1$, then $v_{i}=u$.

Let $M_{H}^{\mu \text { ss }}(v)$ be the moduli space of $S$-equivalence classes of $H$-slopesemistable sheaves of class $v$ (see [HL, Section 8.2]). Then $M_{H}^{\mu \text { ss }}(v)$ is a projective scheme. Set theoretically, $M_{H}^{\mu \text { ss }}(v)$ is the disjoint union of all such varieties $Y(\vec{v}, \vec{d})$. There exists a projective morphism

$$
\bar{\phi}: M_{H}(v) \longrightarrow M_{H}^{\mu \mathrm{ss}}(v)
$$

(see [Li]). Each irreducible component of each fiber of the morphism $\bar{\phi}$ is unirational, as it is dominated by an iterated construction of open subsets in extension bundles and bundles of punctual Quot schemes (see [HL, Theorem 8.2.11]). The morphism $\bar{\phi}$ is thus generically finite, since $M_{H}(v)$ is holomorphic symplectic.

It suffices to prove the inequality

$$
\operatorname{dim} Y(\vec{v}, \vec{d}) \leq \operatorname{dim} M_{H}(v)-4,
$$

for all strata $Y(\vec{v}, \vec{d}) \subset M_{H}^{\mu \text { ss }}(v)$, such that $Y(\vec{v}, \vec{d}) \neq Y_{H}(v)$. It would then follow that $Y_{H}(v)$ is nonempty, and the image of $\bar{\phi}$ is contained in the closure $\bar{Y}_{H}(v)$ of $Y_{H}(v)$ in $M_{H}^{\mu \text { ss }}(v)$. The fiber of $\bar{\phi}$ over a point of $Y_{H}(v)$ consists of a single point. Let $\widetilde{Y}_{H}(v)$ be the normalization of $\bar{Y}_{H}(v)$. The morphism $\bar{\phi}$ would then factor through a surjective birational morphism

$$
\phi: M_{H}(v) \rightarrow \tilde{Y}_{H}(v),
$$


since $M_{H}(v)$ is smooth and irreducible, and Exc $=M_{H}(v)-Y_{H}(v)$ would be the exceptional locus of $\phi$. It would also follow that the singular locus of $\bar{Y}_{H}(v)$ has codimension $\geq 4$ in $\bar{Y}_{H}(v)$. It would then follow that Exc has codimension $\geq 2$ in $M_{H}(v)$, by Proposition 3.5.

Step 2: Upper bounds for $\operatorname{dim} Y(\vec{v}, \vec{d})$. Fix a stratum $Y(\vec{v}, \vec{d})$. Set $t:=t(\vec{v}, \vec{d})$ and $v^{\prime}:=(r, 0, t-s)$. Then $v^{\prime}=\sum_{i=1}^{k} d_{i} v_{i}$. Set

$$
c(\vec{v}, \vec{d}):=\operatorname{dim} M_{H}(v)-\operatorname{dim} Y(\vec{v}, \vec{d}) .
$$

We compute

$$
\begin{aligned}
c(\vec{v}, \vec{d}) & =2+(v, v)-\sum_{i=1}^{k} d_{i}\left[\left(v_{i}, v_{i}\right)+2\right]-2 t \\
& =2+2 t(\epsilon r-1)+\left(v^{\prime}, v^{\prime}\right)-\sum_{i=1}^{k} d_{i}\left[\left(v_{i}, v_{i}\right)+2\right] \\
& =2+2 t(\epsilon r-1)+\sum_{i=1}^{k} \sum_{j=1}^{k} d_{i} d_{j}\left(r_{i} s_{j}+r_{j} s_{i}\right)-2 \sum_{i=1}^{k} d_{i}\left[r_{i} s_{i}+1\right] .
\end{aligned}
$$

Case 1. Suppose that $v_{i} \neq u$, for all $i$. Then $s_{i} \geq r_{i} \geq 2$, for $1 \leq i \leq k$. Write $c(\vec{v}, \vec{d})$ in the form

$$
2+2 t(\epsilon r-1)+2 \sum_{i=1}^{k-1} d_{i} \sum_{j=i+1}^{k} d_{j}\left(r_{i} s_{j}+r_{j} s_{i}\right)+2 \sum_{i=1}^{k} d_{i}\left[\left(d_{i}-1\right) r_{i} s_{i}-1\right] .
$$

Case 1.1. Assume that $k=1$. Then

$$
c(\vec{v}, \vec{d})=2+2 t(\epsilon r-1)+2 d_{1}\left[\left(d_{1}-1\right) r_{1} s_{1}-1\right] .
$$

Case 1.1.1. If $d_{1}=1$, then $c(\vec{v}, \vec{d})=2 t(\epsilon r-1) \geq 4 t$. If $t=0$, we are in the open subset where $F$ is locally free and $H$-slope-stable. If $t>0$, we see that indeed $c(\vec{v}, \vec{d}) \geq 4$.

Case 1.1.2. If $d_{1}>1$, then $2 d_{1}\left[\left(d_{1}-1\right) r_{1} s_{1}-1\right]$ is a positive even number, so $c(\vec{v}, \vec{d}) \geq 4+2 t(\epsilon r-1) \geq 4$.

Case 1.2. Assume that $k>1$. Then

$$
\begin{aligned}
c(\vec{v}, \vec{d}) & =2+2 t(\epsilon r-1)+2(A+B), \quad \text { where } \\
A & =\sum_{i=1}^{k-1} d_{i}\left\{\left[\sum_{j=i+1}^{k} d_{j}\left(r_{i} s_{j}+r_{j} s_{i}\right)\right]+\left[\left(d_{i}-1\right) r_{i} s_{i}-1\right]\right\}, \\
B & =d_{k}\left[\left(d_{k}-1\right) r_{k} s_{k}-1\right] .
\end{aligned}
$$

We are assuming that $s_{i} \geq r_{i} \geq 2$. Hence, $\left(r_{i} s_{j}+r_{j} s_{i}\right) \geq 2 r_{i} r_{j} \geq 8$. Hence, $A \geq 7$. Now $B>0$, if $d_{k}>1$, and $B=-1$, if $d_{k}=1$. The desired inequality $c(\vec{v}, \vec{d}) \geq 4$ follows.

Case 2. Assume that $v_{1}=u$. Note that $r_{1} s_{1}+1=0$ and $r_{1} s_{j}+r_{j} s_{1}=s_{j}-r_{j}=$ $\left(u, v_{j}\right)$. Equation (9.2) becomes

$$
c(\vec{v}, \vec{d})=A+B+C, \quad \text { where }
$$




$$
\begin{aligned}
& A=2+2 t(\epsilon r-1)-2 d_{1}^{2} \\
& B=2 d_{1} \sum_{j=2}^{k} d_{j}\left(s_{j}-r_{j}\right)=2 d_{1}\left(u, v^{\prime}-d_{1} v_{1}\right)=2 d_{1}\left(2 d_{1}+s-r-\epsilon t\right), \\
& C=\sum_{i=2}^{k} \sum_{j=2}^{k} d_{i} d_{j}\left(r_{i} s_{j}+r_{j} s_{i}\right)-2 \sum_{i=2}^{k} d_{i}\left[r_{i} s_{i}+1\right] .
\end{aligned}
$$

Note the equality

$$
A+B=2+2 t\left(\epsilon r-\epsilon d_{1}-1\right)+2 d_{1}\left(s-r+d_{1}\right) .
$$

Case 2.1. Assume that $k=1$. Then $r=d_{1}, t=(r+s) / \epsilon$, and

$$
\begin{aligned}
c(\vec{v}, \vec{d}) & =A=2+2\left[(r+s)(r-(1 / \epsilon))-r^{2}\right] \\
& \geq 2+2[(s-1)(r-1)-1] \geq 2 r(r-1) \geq 12 .
\end{aligned}
$$

Case 2.2. Assume that $k \geq 2$. Then $1 \leq \epsilon d_{1} \leq \epsilon r-d_{2} r_{2} \leq \epsilon r-2$. So

$$
\begin{aligned}
A+B \geq & 2+2 t+2 d_{1}(s-r+1) \geq 2+4 d_{1} \geq 6, \\
C / 2= & \sum_{i=2}^{k-1} \sum_{j=i+1}^{k} d_{i} d_{j}\left(r_{i} s_{j}+r_{j} s_{i}\right)+\sum_{i=2}^{k}\left[d_{i}^{2} r_{i} s_{i}-d_{i}\left(r_{i} s_{i}+1\right)\right] \\
= & \sum_{i=2}^{k-1} d_{i}\left(\left[\sum_{j=i+1}^{k} d_{j}\left(r_{i} s_{j}+r_{j} s_{i}\right)\right]+\left[\left(d_{i}-1\right) r_{i} s_{i}\right]-1\right) \\
& +d_{k}\left[\left(d_{k}-1\right) r_{k} s_{k}-1\right] .
\end{aligned}
$$

If $k=2$ and $d_{2}=1$, then $C=-2$. Otherwise, $C \geq 0$. We conclude that $c(\vec{v}, \vec{d}) \geq 4$. This completes the proof of Lemma 9.5.

\subsubsection{The case with slope equal to one half}

Let $r$ be a positive odd integer, $\sigma$ a positive integer, and set $n:=r \sigma+1$. Assume that $r \geq 3, \sigma \geq 3$, and $\operatorname{gcd}(r, \sigma)=1$. Let $S$ be a $K 3$ surface with a cyclic Picard group generated by an ample line bundle $H$. Set $d:=\operatorname{deg}(H) / 2$. Choose $(S, H)$, so that $\sigma$ and $d$ have the same parity. If $d$ is odd, assume that $\sigma>r$, possibly after interchanging $r$ and $\sigma$. Set $h:=c_{1}(H)$ and $v:=(2 r, r h,-b)$, where $b:=[\sigma-r d] / 2$. Note that $\operatorname{gcd}(r, b)=\operatorname{gcd}(r, \sigma)=1$. Hence, $v$ is a primitive class in $K(S),(v, v)=$ $2 n-2$, and the moduli space $M_{H}(v)$ is smooth and projective of type $K 3^{[n]}$. Let Exc $\subset M_{H}(v)$ be the locus parameterizing sheaves $F$ that are not locally free or not $H$-slope-stable.

\section{LEMMA 9.6}

Exc is an algebraic subset of codimension ${ }^{*} \geq 2$ in $M_{H}(v)$.

*Note that the assumption $\sigma>r$, adopted above when $d$ is odd, is necessary, since otherwise $\operatorname{Exc}=M_{H}(v)$, by Lemma 9.3. 
Proof

Proof in the case $d$ is odd. When $d$ is odd, then $\sigma$ is odd. Set $u:=(2, h,(d+1) / 2)$ and $s:=r+(v, u)$. Then $s=\sigma$ is odd. Thus, $s>r$, by assumption, and $s-r$ is even, so $s \geq r+2$.

Given an $H$-slope-stable locally free sheaf $F_{i}$ of class $v_{i}=\left(2 r_{i}, r_{i} h,-b_{i}\right)$, set $s_{i}:=r_{i}+\left(v_{i}, u\right)$. If $v_{i}=u$, then $s_{i}=-1$. If $v_{i} \neq u$, then $s_{i} \geq r_{i}$, by Lemma 9.3. Furthermore, $s_{i}=r_{i}$ if and only if $v_{i}=(2, h,(d-1) / 2)$. If $v_{i} \neq u$ and $s_{i} \neq r_{i}$, then $s_{i} \geq r_{i}+2$, since $s_{i}-r_{i}$ is even, by Lemma 9.3 .

With the above notation of $s$ and $s_{i}$, the proof is almost identical to that of Lemma 9.5. Following are the necessary changes. Replace the class $(1,0,1)$ by the class $u$ defined above. Then $\epsilon=\operatorname{rank}(u)=2$. Set $\lambda:=(0,0,-1)$. Then $(u, \lambda)=2$ and $(\lambda, \lambda)=0$. With the above definition of $s_{i}$, we have $v_{i}=r_{i} u+\left(\left(s_{i}+r_{i}\right) / 2\right) \lambda$. Hence,

$$
\left(v_{i}, v_{j}\right)=r_{i} s_{j}+r_{j} s_{i}
$$

as in the proof of Lemma 9.5.

Equation (9.1) is replaced by

$$
r_{i}=-s_{i}=1, \quad s_{i}=r_{i}=1, \quad \text { or } \quad s_{i} \geq r_{i}+2 \geq 3,
$$

by Lemma 9.3. Equation (9.2), for the codimension $c(\vec{v}, \vec{d})$ of $Y(\vec{v}, \vec{d})$, remains valid. The argument for case 1.1.1 remains valid. In case 1.1.2 the term $2 d_{1}\left[\left(d_{1}-\right.\right.$ 1) $\left.r_{1} s_{1}-1\right]$ vanishes, if $d_{1}=2$ and $r_{1}=s_{1}=1$. However, in that case $\left(u, v^{\prime}\right)=$ $\left(u, 2 v_{1}\right)=0$ and $2 r t=\left(u, v-v^{\prime}\right)=(u, v)=s-r>0$. So $t>0$ and $c(\vec{v}, \vec{d})=2+$ $2 t(2 r-1) \geq 4$.

In case 1.2 we are no longer assuming that $r_{i} \geq 2$. However, since all $v_{i}$ are different from $u$, and $k>1$, then at least one $v_{i}$, say, $v_{1}$, is different from $(2, h,(d+1) / 2)$. Then $s_{1} \geq r_{1}+2 \geq 3$. Thus $r_{1} s_{2}+r_{2} s_{1} \geq 4$ and $A \geq 3$. The rest of the argument in case 1.2 is identical.

In case 2 , the equations for $c(\vec{v}, \vec{d}), A, B$, and $C$, remain valid. The argument in case 2.1 remains valid. The inequality $\epsilon r-d_{2} r_{2} \leq \epsilon r-2$, in the first line of case 2.2 , need not hold. Nevertheless, $\epsilon r-\epsilon d_{1} \geq \epsilon d_{2} \geq 2$. So

$$
A+B \geq 2+2 d_{1}(s-r+1) \geq 2+6 d_{1} \geq 8 .
$$

The rest of the argument remains valid.

Proof in the case $d$ is even*. When $d$ is even, then $\sigma$ is even. Set $u:=$ $(2, h, d / 2)$. By assumption, $r \geq 3, s \geq 3$, and $\operatorname{gcd}(r, s)=1$.

Given an $H$-slope-stable sheaf $F_{i}$ of class $v_{i}=\left(2 r_{i}, r_{i} h,-b_{i}\right)$, with $r_{i}>0$, set $\sigma_{i}:=\left(u, v_{i}\right)=2 b_{i}+r_{i} d$. If $v_{i}=u$, then $\sigma_{i}=0$. If $v_{i} \neq u$, then $\sigma_{i}$ is a positive even integer, by Lemma 9.4. Note also that $(u, v)=\sigma$.

The proof is again almost identical to that of Lemma 9.5. Following are the necessary changes. Replace the class $(1,0,1)$ by the class $u$ defined above. Then $\epsilon=\operatorname{rank}(u)=2$. Set $\lambda:=(0,0,-1)$. Then $(u, \lambda)=2$ and $(\lambda, \lambda)=0$. With the 
above definition of $\sigma_{i}$, we have $v_{i}=r_{i} u+\left(\sigma_{i} / 2\right) \lambda$. Hence,

$$
\left(v_{i}, v_{j}\right)=r_{i} \sigma_{j}+r_{j} \sigma_{i},
$$

and we replace $s_{i}$ by $\sigma_{i}$ in the proof of Lemma 9.5. Then Equation (9.2), for the codimension $c(\vec{v}, \vec{d})$ of $Y(\vec{v}, \vec{d})$, remains valid.

Equation (9.1) is replaced by

$$
v_{i}=u \quad \text { or } \quad \sigma_{i} \text { is a positive even integer, }
$$

by Lemma 9.4. The argument for case 1.1 remains valid.

In case 1.2 we are no longer assuming that $r_{i} \geq 2$. However, since all the $v_{i}$ are different from $u$, then $\sigma_{i} \geq 2$, for all $i$. Thus, $r_{1} \sigma_{2}+r_{2} \sigma_{1} \geq 4$ and $A \geq 3$. The rest of the argument is identical.

In case $2, v_{1}=u, \sigma_{1}=0$ and $\left(r_{1} \sigma_{j}+r_{j} \sigma_{1}\right)=\sigma_{j}=\left(u, v_{j}\right)$. Then $c(\vec{v}, \vec{d})=$ $A+B+C$, where

$$
\begin{aligned}
& A=2+2 t(\epsilon r-1), \\
& B=2 d_{1} \sum_{j=2}^{k} d_{j} \sigma_{j}=2 d_{1}\left(u, v^{\prime}-d_{1} v_{1}\right)=2 d_{1}(\sigma-\epsilon t),
\end{aligned}
$$

and $C$ remains the same. Then

$$
A+B=2+2 t\left(\epsilon r-\epsilon d_{1}-1\right)+2 d_{1} \sigma .
$$

In case 2.1,r $=d_{1}, v^{\prime}=r u, \epsilon t=\left(v-v^{\prime}, u\right)=\sigma$, and $c(\vec{v}, \vec{d})=A=2+\sigma(\epsilon r-1)$. Hence $c(\vec{v}, \vec{d}) \geq 17$.

In case 2.2 we assume that $k \geq 2$ and so $\epsilon\left(r-d_{1}\right) \geq 2$. So $A+B \geq 2+$ $2 d_{1} \sigma \geq 8$. The rest of the argument is the same. This completes the proof of Lemma 9.6.

\section{Examples of prime exceptional divisors}

Let $e, E$, and $X$ be as in Theorem 1.2. Set $n:=\operatorname{dim}_{\mathbb{C}}(X) / 2$. The pair $(X, E)$ has the following elementary invariants:

(1) $(e, e)=-2$, or $(e, e)=2-2 n$;

(2) the divisibility $\operatorname{div}(e, \bullet)$ of the class $(e, \bullet)$ in $H^{2}(X, \mathbb{Z})^{*}$ is equal to $(e, e)$ or $(e, e) / 2$;

(3) write $[E]=k e$, where $e$ is a primitive class in $H^{2}(X, \mathbb{Z})$; then $k=1$, or $k=2$.

Set $[E]^{\vee}:=-2([E], \bullet) /([E],[E])$. We have $[E]^{\vee}=e^{\vee}$ or $[E]^{\vee}=2 e^{\vee}$, where $e^{\vee}$ is a primitive class in $H_{2}(X, \mathbb{Z})$, and the coefficient is determined by Lemma 3.7 in terms of the invariant $\operatorname{div}(e, \bullet)$ and the coefficient $k$ in (3) above. In particular, if $\operatorname{div}(e, \bullet)=(e, e) / 2$, then $[E]=e$, by Lemma 3.7 .

\section{THEOREM 10.1}

Let $X$ be a smooth projective holomorphically symplectic variety, and let $E$ be a prime divisor on $X$. 
(1) Assume that through a generic point of $E$ passes a rational curve of class $\ell \in H_{2}(X, \mathbb{Z})$, such that $[E] \cdot \ell<0$. Then $E$ is an exceptional divisor (see $[D$, Theorem 1.3]).

(2) Let $E$ and $\ell$ be as in part (1), and let $\pi: X^{\prime} \rightarrow Y$ be the birational contraction of $E$ introduced in Proposition 3.1. Then $Y$ has $A_{i}$-singularities away from its dissident locus, ${ }^{*}$ and $i=1$ or $i=2$. Furthermore,

$$
\ell= \begin{cases}{[E]^{\vee}} & \text { if } i=1, \\ \frac{1}{2}[E]^{\vee} & \text { if } i=2 .\end{cases}
$$

Proof

We need only prove part (2). $Y$ has $A_{1}$ - or $A_{2}$-singularities, by Corollary 3.6. Let $E^{\prime}$ be the strict transform of $E$ in $X^{\prime}$. The generic fiber of the restriction of $\pi$ to $E^{\prime}$ is a rational curve, or a pair of rational curves joined at a node. The exceptional locus of the birational transformation from $X$ to $X^{\prime}$ does not dominate $\pi\left(E^{\prime}\right)$, by the proof of [D, Proposition 1.4]. The morphism $\pi$ thus restricts to a rational morphism from $E$ to $\pi\left(E^{\prime}\right)$, whose generic fiber is isomorphic to the generic fiber of $E^{\prime}$ over $\pi\left(E^{\prime}\right)$. The class $\ell$ must be the class of an irreducible component of the generic fiber of the restriction of $\pi$ to $E$, by the uniqueness of the family of rational curves, which dominates $E$ (see [D, Proposition 4.5]). The equality $\ell=(1 / i)[E]^{\vee}$ follows from Corollary 3.6(1).

We will say that the prime exceptional divisor is of type $A_{i}$, if the variety $Y$ in Theorem 10.1(2) has $A_{i}$-singularities away from its dissident locus. All prime exceptional divisor studied in this paper are of type $A_{1}$.

\subsection{Brill-Noether exceptional divisors}

Let $S$ be a $K 3$ surface, let $F_{0}$ be a simple and rigid coherent sheaf, that is, a sheaf satisfying $\operatorname{End}\left(F_{0}, F_{0}\right) \cong \mathbb{C}$ and $\operatorname{Ext}^{1}\left(F_{0}, F_{0}\right)=0$. Then the class $v_{0}$ of $F_{0}$ is a primitive class in $K(S)$ with $\left(v_{0}, v_{0}\right)=-2$. Examples of exceptional divisors $E$ of degree -2 in moduli spaces of sheaves on $S$ seem to arise as Brill-Noether loci as follows. Let $v \in K(S)$ be a class satisfying $\left(v_{0}, v\right)=0$ and such that there exists a smooth and compact moduli space $M(v)$ of stable sheaves of class $v$. The locus $M(v)^{1}$, of points representing sheaves $F$ with nonvanishing $\operatorname{Ext}^{1}\left(F, F_{0}\right)$, is often an exceptional divisor of degree -2 . The examples considered in this section are all of this type.

\section{EXAMPLE 10.2}

An example is the case $n \geq 2,(e, e)=-2$, and $\operatorname{div}(e, \bullet)=1$. Let $S$ be a $K 3$ surface containing a smooth irreducible rational curve $\Sigma$. Let $E \subset S^{[n]}, n \geq 2$, be the divisor consisting of length $n$ subschemes intersecting $\Sigma$ along a nonempty subscheme. The class $[E]$ is identified with $[\Sigma]$, under the embedding of $H^{2}(S, \mathbb{Z})$ as 
an orthogonal direct summand in the decomposition (4.1) of $H^{2}\left(S^{[n]}, \mathbb{Z}\right)$. Thus $([E],[E])=([\Sigma],[\Sigma])=-2$ and $\operatorname{div}([E], \bullet)=1$. $E$ is of type $A_{1}$. Let $F_{0}$ be the direct image of $\mathcal{O}_{\Sigma}(-1)$, and let $v_{0} \in K(S)$ be the class of $F_{0}$. Then $v_{0}$ is orthogonal to the class of the ideal sheaf $I_{Z}$ of a length $n$ subscheme $Z$ of $S$, and $E$ is the Brill-Noether locus, where $\operatorname{Ext}^{1}\left(I_{Z}, F_{0}\right)$ does not vanish.

Let $S$ be a projective $K 3$ surface, with a cyclic Picard group generated by an ample line bundle $H$ of degree $d \geq 2$. In the remainder of this section the simple and rigid sheaf $F_{0}$ will be $\mathcal{O}_{S}$. Then $\operatorname{Ext}^{1}\left(F, \mathcal{O}_{S}\right) \cong H^{1}(F)^{*}$, by Serre's duality. We will need the following results.

LEMMA 10.3 ([Ma1, LEMMA 3.7(3)])

Let $F$ be an $H$-stable sheaf on $S$ of rank $r$ and determinant $H$, and let $U \subset$ $H^{0}(F)$ be a subspace of dimension $r^{\prime} \leq r$. Then the evaluation homomorphism $U \otimes \mathcal{O}_{S} \rightarrow F$ is injective and its cokernel is an $H$-stable sheaf.

Consider the Mukai vector $v:=(r, H, s)$, and assume that $r \geq 0$ and $r+s \geq 0$. Set $v_{0}:=(1,0,1)$. Let $M_{H}(v)^{t}$ be the Brill-Noether locus of $H$-stable sheaves $F$ with $h^{1}(F) \geq t$.

THEOREM 10.4 ([Ma1, COROLLARY 3.19], [Y1])

(1) $M_{H}(v)^{t}$ is empty if and only if $M_{H}\left(v+t v_{0}\right)$ is.

(2) There exists a smooth surjective projective morphism

$$
f_{t}:\left[M_{H}(v)^{t} \backslash M_{H}(v)^{t+1}\right] \longrightarrow\left[M_{H}\left(v+t v_{0}\right) \backslash M_{H}\left(v+t v_{0}\right)^{1}\right] .
$$

(3) The fiber of $f_{t}$, over a point representing a sheaf $E$, is naturally isomorphic to the Grassmannian $G\left(t, H^{0}(E)\right)$. Furthermore, $H^{0}(E)$ is $(r+s+2 t)$ dimensional, and the dimension $t(r+s+t)$ of the fiber is equal to the codimension of $M_{H}(v)^{t}$ in $M_{H}(v)$.

(4) If $s=-r$, then $M(v)^{1}$ is a prime divisor of class $\theta\left(-v_{0}\right)$. The class $\ell \in H^{2}\left(M_{H}(v), \mathbb{Z}\right)^{*}$ of a $\mathbb{P}^{1}$-fiber of $f_{1}$ is $\left(\theta\left(-v_{0}\right), \bullet\right)$ (see [Ma\%, Lemma 4.11]).

The embedding $G\left(t, H^{0}(E)\right) \hookrightarrow M_{H}(v)$ in part (3) sends a $t$-dimensional subspace $U \subset H^{0}(E)$ to the cokernel of the evaluation homomorphism $U \otimes \mathcal{O}_{S} \rightarrow E$. The cokernel is stable, by Lemma 10.3 .

\section{EXAMPLE 10.5}

The case $n \geq 2,(e, e)=-2$, and $\operatorname{div}(e, \bullet)=1$ was considered in Example 10.2. Additional examples of such prime exceptional divisors are provided in Theorem 10.4(4). $M_{H}(v)^{1}$ is exceptional, since it is prime of degree -2 , by Theorem 10.4(4). Examples of prime exceptional Brill-Noether divisors, for more general simple and rigid sheaves, can be found in the work of Yoshioka [Y1]. 


\section{EXAMPLE 10.6}

An example is the case $(e, e)=-2, \operatorname{div}(e, \bullet)=2$, and $[E]=e$.

Assume that $n$ is congruent to 2 modulo 4 and $n \geq 6$. Let $S$ be a projective $K 3$ surface with a cyclic Picard group generated by an ample line bundle $H$ of degree $(H, H)=(n-2) / 2$. Then $h^{i}\left(H^{2}\right)=0$, for $i>0$, and $h^{0}\left(H^{2}\right)=n$. Set $X:=$ $M_{H}\left(1, H^{2},-1\right) \cong S^{[n]}$. Let $E:=M_{H}\left(1, H^{2},-1\right)^{1}$ be the Brill-Noether divisor in $M_{H}\left(1, H^{2},-1\right)$ of sheaves $F$ with $h^{1}(S, F)>0$.

We recall the explicit definition of $E$. Let $\pi_{i}, i=1,2$, be the projection from $S \times M_{H}\left(1, H^{2},-1\right)$ onto the $i$ th factor. Let $\mathcal{Z} \subset S \times M_{H}\left(1, H^{2},-1\right)$ be the universal subscheme, and let $I_{\mathcal{Z}}$ be its ideal sheaf. Then $\mathcal{F}:=I_{\mathcal{Z}} \otimes \pi_{1}^{*} H^{2}$ is a universal sheaf over $S \times \mathcal{M}_{H}\left(1, H^{2},-1\right)$. We have the short exact sequence

$$
0 \rightarrow \mathcal{F} \rightarrow \pi_{1}^{*} H^{2} \rightarrow \mathcal{O}_{\mathcal{Z}} \otimes \pi_{1}^{*} H^{2} \rightarrow 0
$$

and the homomorphism of rank $n$ vector bundles

$$
g: H^{0}\left(H^{2}\right) \otimes \mathcal{O}_{M_{H}(v)} \cong \pi_{2_{*}}\left(\pi_{1}^{*} H^{2}\right) \longrightarrow \pi_{2_{*}}\left(\mathcal{O}_{\mathcal{Z}} \otimes \pi_{1}^{*} H^{2}\right) .
$$

The homomorphism $g$ is injective, since a generic length $n$ subscheme of $S$ induces $n$ independent conditions on a linear system $|L|$, provided the line bundle $L$ on $S$ satisfies $h^{0}(L) \geq n$. The Brill-Noether divisor is the zero divisor of $\wedge^{n} g$. $E$ is an effective divisor of class $\theta\left(-v_{0}\right)$, where $v_{0}:=(1,0,1)$ is the class in $K(S)$ of the trivial line bundle, and $\theta$ is the Mukai isomorphism given in (2.2).

\section{LEMMA 10.7}

$E$ is a prime exceptional divisor of class $e:=\theta\left(-v_{0}\right)$. In particular, $(e, e)=-2$ and $\operatorname{div}(e, \bullet)=2$.

The rest of Section 10.1 is devoted to the proof of Lemma 10.7.

LEMMA 10.8

Let $F$ be an $H$-slope-stable sheaf of class $\left(2, H^{2}, 0\right)$.

(1) For every nonzero section $s \in H^{0}(F)$, the evaluation homomorphism $s$ : $\mathcal{O}_{S} \rightarrow F$ has a rank 1 torsion-free cokernel sheaf.

(2) If $\epsilon \in \operatorname{Ext}^{1}\left(\mathcal{O}_{S}, F\right)$ is a nonzero class and

$$
0 \rightarrow \mathcal{O}_{S} \rightarrow G_{\epsilon} \rightarrow F \rightarrow 0
$$

is the corresponding extension, then the sheaf $G_{\epsilon}$ is $H$-slope-stable.

\section{Proof}

(1) Denote the cokernel of $s$ by $Q_{s}$. If $T$ is a subsheaf of $Q_{s}$ with zero-dimensional support, then $\operatorname{Ext}^{1}\left(T, \mathcal{O}_{S}\right)=0$. Thus, the inverse image of $T$ in $F$ would contain a subsheaf isomorphic to $T$. But $F$ is torsion-free. Hence, the dimension of the support of any subsheaf of $Q_{s}$ is at least 1 .

If $T$ is a subsheaf of $Q_{s}$ of one-dimensional support, then its inverse image in $F$ is a rank one subsheaf $F^{\prime}$ of $F$ with effective determinant line bundle. Hence, 
$\operatorname{det}\left(F^{\prime}\right) \cong H^{k}$, for some positive integer $k$. This contradicts the slope stability of $F$. Hence, $Q_{s}$ is torsion-free.

(2) Assume that $G_{\epsilon}$ is $H$-slope-unstable, and let $G^{\prime} \subset G_{\epsilon}$ be an $H$-slopestable subsheaf of maximal slope of rank $r \leq 2$. If $G^{\prime}$ maps to zero in $F$, then $G^{\prime}$ is a subsheaf of $\mathcal{O}_{S}$ and cannot destabilize $G_{\epsilon}$. For the same reason, the slope of the image $\bar{G}$ of $G^{\prime}$ satisfies $\mu(\bar{G}) \geq \mu\left(G^{\prime}\right)$. Thus $\operatorname{rank}(\bar{G}) \neq 1$, since otherwise $\bar{G}$ would destabilize $F$. Hence, $\operatorname{rank}\left(G^{\prime}\right)=2, G^{\prime}$ maps isomorphically onto $\bar{G}$, and $\operatorname{det}\left(G^{\prime}\right) \cong H^{k}$, for $2 / 3<k \leq 2$. It follows that $k=2$.

Set $Q:=F / \bar{G}$. We get the short exact sequence

$$
0 \rightarrow \bar{G} \stackrel{\iota}{\rightarrow} F \rightarrow Q \rightarrow 0 .
$$

$\operatorname{Ext}^{1}\left(Q, \mathcal{O}_{S}\right)$ vanishes, since $Q$ has zero-dimensional support. Hence, $\iota^{*}: \operatorname{Ext}^{1}(F$, $\left.\mathcal{O}_{S}\right) \rightarrow \operatorname{Ext}^{1}\left(\bar{G}, \mathcal{O}_{S}\right)$ is injective. On the other hand, the pullback $\iota^{*}(\epsilon)$ vanishes in $\operatorname{Ext}^{1}\left(\bar{G}, \mathcal{O}_{S}\right)$. This contradicts the assumption that $\epsilon$ is a nonzero class.

The moduli space $M_{H}^{\text {ss }}\left(2, H^{2}, 0\right)$, of $H$-semistable sheaves of class $\left(2, H^{2}, 0\right)$, is known to be an irreducible normal projective variety of dimension $2 n-2$. Furthermore, the singular locus is equal to the strictly semistable locus, and it has codimension 2, if $n=6$, and 4 , if $n>6$ (see [KLS, Theorems 4.4, 5.3]). A generic $H$-stable sheaf of class $\left(2, H^{2}, 0\right)$ is $H$-slope-stable. This is equivalent to the corresponding statement for $M_{H}(2,0,1-(n / 2))$ and follows from the next lemma.

\section{LEMMA 10.9}

Let $s$ be an integer $\geq 2$. Then the set of $H$-stable locally free sheaves of class $(2,0,-s)$ is Zariski dense in $M_{H}^{\mathrm{ss}}(2,0,-s)$. Furthermore, any $H$-stable locally free sheaf of class $(2,0,-s)$ is $H$-slope-stable.

The proof of the density statement is similar to that of Lemma 9.5 and is omitted. The case $s=2$ is proven in [OG2, Proposition 3.0.5]. The second statement is a special case of Lemma 9.1.

LEMMA 10.10

Let $U \subset M_{H}^{\text {ss }}\left(2, H^{2}, 0\right)$ be the subset parameterizing $H$-slope-stable sheaves $F$ with $h^{1}(F)=0$. Then $U$ is a Zariski-dense open subset.

Proof

Let $M^{\mu s} \subset M_{H}^{\text {ss }}\left(2, H^{2}, 0\right)$ be the Zariski open subset of $H$-slope-stable sheaves. Note that $M^{\mu s}$ is a dense subset, by Lemma 10.9. Let $t$ be the minimum of the set $\left\{h^{1}(F):[F] \in M^{\mu s}\right\}$. It suffices to prove that $t=0$. Assume that $t>0$. Let $U^{\prime} \subset M^{\mu s}$ be the Zariski open subset of sheaves $F$ with $h^{1}(F)=t$. Let $p: \mathbb{P} \rightarrow U^{\prime}$ be the projective bundle with fiber $\mathbb{P} H^{1}(F)^{*}$ over $F . \mathbb{P}$ is a Zariski open subset of the moduli space of coherent systems constructed by Le Potier in [Le]. A point 
in $\mathbb{P}$ parameterizes an equivalence class of a pair $(F, \ell)$, consisting of an $H$-slopestable sheaf $F$ of class $\left(2, H^{2}, 0\right)$ and a one-dimensional subspace $\ell \subset \operatorname{Ext}^{1}\left(F, \mathcal{O}_{S}\right)$. We have $\operatorname{dim}(\mathbb{P})=\operatorname{dim}\left(U^{\prime}\right)+t-1=2 n+t-3$.

There exists a natural morphism

$$
f: \mathbb{P} \longrightarrow M_{H}\left(3, H^{2}, 1\right),
$$

sending a pair $(F, \ell)$ to the isomorphism class of the sheaf $G_{\ell}$ in the natural extension $0 \rightarrow \ell^{*} \otimes \mathcal{O}_{S} \rightarrow G_{\ell} \rightarrow F \rightarrow 0$. $G_{\ell}$ is $H$-slope-stable, by Lemma $10.8(2)$.

Now $h^{0}\left(G_{\ell}\right)=h^{0}(F)+1=t+3$. Furthermore, the data $(F, \ell)$ is equivalent to the data $\left(G_{\ell}, \ell\right)$, where $\ell$ is a one-dimensional subspace of $H^{0}\left(G_{\ell}\right)$. Hence, the fiber of $f$, over the isomorphism class of $G_{\ell}$, has dimension at most $t+2$. The dimension of $M_{H}\left(3, H^{2}, 1\right)$ is $2 n-8$. Thus, $\operatorname{dim}(\mathbb{P}) \leq 2 n+t-6$. This contradicts the above computation of the dimension of $\mathbb{P}$.

Let $G(1, U)$ be the moduli space of equivalence classes of pairs $(F, \lambda)$, where $F$ is an $H$-slope-stable sheaf of class $\left(2, H^{2}, 0\right)$ with $h^{1}(F)=0$, and $\lambda \subset H^{0}(F)$ is a one-dimensional subspace. $G(1, U)$ is a Zariski open subset of the moduli space of coherent systems constructed by Le Potier in [Le]. The forgetful morphism $G(1, U) \rightarrow U$ is a $\mathbb{P}^{1}$-bundle. Let

$$
\psi: G(1, U) \longrightarrow M_{H}\left(1, H^{2},-1\right)
$$

be the morphism sending a pair $(F, \lambda)$ to the quotient $F /\left[\lambda \otimes \mathcal{O}_{S}\right]$. The morphism $\psi$ is well defined, by Lemma $10.8(1)$.

\section{LEMMA 10.11}

(1) The divisor $E$ is smooth along the image of $\psi$, and $\psi$ maps $G(1, U)$ isomorphically onto a Zariski open subset of $E$.

(2) Let $F$ be an $H$-slope-stable sheaf of class $\left(2, H^{2}, 0\right)$ with $h^{1}(F)=0$. Then $\psi\left(\mathbb{P} H^{0}(F)\right)$ is a rational curve of class $\left(\theta\left(-v_{0}\right), \bullet\right)$ in $H_{2}\left(M_{H}\left(1, H^{2},-1\right), \mathbb{Z}\right)$.

\section{Proof}

(1) The proof is similar to that of [Ma1, Proposition 3.18(6), (7)]. Let us first prove that the morphism $\psi$ is injective. Let $Q$ be a sheaf represented by the point $\psi(F, \lambda)$. We know, by construction, that $H^{i}(F)=0$, for $i>0$, and $h^{0}(F)=$ $\chi(F)=2$. Hence, $h^{0}(Q)=1, h^{1}(Q)=1$, and $h^{2}(Q)=0$. It follows that $\operatorname{dim} \operatorname{Ext}^{1}\left(Q, \mathcal{O}_{S}\right)=1, F$ is isomorphic to the unique nontrivial extension of $Q$ by $\mathcal{O}_{S}$, and $\lambda$ is the kernel of the homomorphism $H^{0}(F) \rightarrow H^{0}(Q)$. Hence, $\psi$ is injective.

The image of $\psi$ is Zariski open in $E$, since it is characterized by $\operatorname{dim} \operatorname{Ext}^{1}\left(Q, \mathcal{O}_{S}\right)=1$ and by the $H$-slope-stability of the unique nontrivial extension. $G(1, U)$ is clearly smooth. It suffices to construct the inverse of $\psi$ as a morphism. This is done as in the proof of [Ma1, Proposition 3.18]. 
(2) Let $w \in K(S)$ be a class orthogonal to $\left(1, H^{2},-1\right)$. The equality

$$
\int_{\mathbb{P} H^{0}(F)} \psi^{*}(\theta(w))=-\left(v_{0}, w\right)
$$

follows by an argument identical to the proof of [Ma7, Lemma 4.11]. It follows that $\psi\left(\mathbb{P} H^{0}(F)\right)$ has class $\left(\theta\left(-v_{0}\right), \bullet\right)$ in $H_{2}\left(M_{H}\left(1, H^{2},-1\right), \mathbb{Z}\right)$.

\section{LEMMA 10.12}

The closure $E^{\prime}$ of the image of $\psi$ is a prime exceptional divisor of class $\theta\left(-v_{0}\right)$.

\section{Proof}

$G(1, U)$ is a $\mathbb{P}^{1}$-bundle over $U$. Hence $G(1, U)$ is irreducible of dimension $2 n-1$. The image of $\psi$ is irreducible of dimension $2 n-1$, as $\psi$ is injective. Hence, $E^{\prime}$ is irreducible. The canonical line bundle of $G(1, U)$ restricts to the fiber $\mathbb{P} H^{0}(F)$ as the canonical line bundle of the fiber, since $U$ is holomorphic symplectic. The normal of $\psi(G(1, U))$ in $M_{H}\left(1, H^{2},-1\right)$ is isomorphic to the canonical line bundle of $\psi(G(1, U))$, by Lemma $10.11(1)$. Hence, $E^{\prime} \cdot \psi\left[\mathbb{P} H^{0}(F)\right]=-2$, and $E^{\prime}$ is exceptional, by Theorem 10.1(1). $E^{\prime}$ is of type $A_{1}$, by Lemma 10.11(1). The class of $E^{\prime}$ is $\theta\left(-v_{0}\right)$, by Lemma 10.11 and Theorem $10.1(2)$.

\section{Proof of Lemma 10.7}

$E$ is an effective divisor of class $\theta\left(-v_{0}\right)$, by definition of $E$. This is also the class of the reduced and irreducible divisor $E^{\prime}$ supporting a component of $E$. Hence, $E$ is reduced and irreducible. We have the equality $([E], \theta(x))=\left(\theta\left(-v_{0}\right), \theta(x)\right)=$ $-\left(v_{0}, x\right)$, which is divisible by 2 , for all $x \in\left(1, H^{2},-1\right)^{\perp}$, since $\left(1, H^{2},-1\right)-v_{0}=$ $2(0, H,-1)$. Hence, $\operatorname{div}([E], \bullet)=2$.

\subsection{Exceptional divisors of nonlocally free sheaves}

In this section we will consider examples of prime exceptional divisors that arise as the exceptional locus for the morphism from the Gieseker-Simpson moduli space of $H$-stable sheaves to the Uhlenbeck-Yau compactification of the moduli space of $H$-slope-stable locally free sheaves. Such divisors on a $2 n$-dimensional moduli space seem to have class $e$ or $2 e$, where $e$ is a primitive class of degree $(e, e)=2-2 n$.

\section{EXAMPLE 10.13 ([Be1])}

An example is the case $n \geq 2,(e, e)=2-2 n, \operatorname{div}(e, \bullet)=2 n-2, r s(e)=\{1, n-1\}$, and $[E]=2 e$.

Let $S$ be a $K 3$ surface, let $X:=S^{[n]}$, and let $E \subset X$ be the big diagonal. Then $[E]=2 e$, for a primitive class $e \in H^{2}\left(S^{[n]}, \mathbb{Z}\right)$, and $(e, e)=2-2 n$. Hence $[E]^{\vee}=e^{\vee}$, by Corollary 3.6. $E$ is the exceptional locus of the Hilbert-Chow morphism $S^{[n]} \rightarrow S^{(n)}$ onto the $n$th symmetric product. The symmetric product $S^{(n)}$ has $A_{1}$-singularities away from its dissident locus. The monodromy invariant $r s(e)$ is equal to $\{1, n-1\}$, by Example 7.2. 
The following result will be needed in the next example.

\section{LEMMA 10.14}

Let $S$ be a $K 3$ surface, let $\mathcal{L}$ be a line bundle on $S$, let $v=(r, \mathcal{L}, s)$ be a class in $K(S)$ satisfying $(v, v) \geq 2$, and let $r \geq 2$. Let $H$ be a $v$-generic polarization. Assume given an $H$-slope-stable sheaf $G$ of class $(r, \mathcal{L}, s+1)$ and a point $P \in S$, such that $G$ is locally free at $P$. For each 2-dimensional quotient $Q$ of the fiber $G_{P}$, there exists a natural embedding

$$
\kappa: \mathbb{P} Q \longrightarrow M_{H}(v),
$$

whose image $C:=\kappa(\mathbb{P} Q)$ is a smooth rational curve satisfying

$$
\int_{C} \theta(x)=(w, x)=-\operatorname{rank}(x),
$$

for all $x \in v^{\perp}$, where $w$ is the following rational class in $v^{\perp}$ :

$$
w:=\frac{r}{(v, v)} v+(0,0,1)=\frac{1}{(v, v)}\left(r^{2}, r \mathcal{L}, s r+(v, v)\right) .
$$

Proof

Consider the short exact sequence of the tautological quotient bundle $q_{\mathbb{P} Q}$ over $\mathbb{P} Q$,

$$
0 \rightarrow \mathcal{O}_{\mathbb{P} Q}(-1) \rightarrow Q \otimes \mathcal{O}_{\mathbb{P} Q} \rightarrow q_{\mathbb{P} Q} \rightarrow 0
$$

Let

$$
\iota: \mathbb{P} Q \rightarrow S \times \mathbb{P} Q
$$

be the morphism given by $\iota(\ell)=(P, \ell)$. Let $\pi_{i}$ be the projection from $S \times \mathbb{P} Q$ onto the $i$ th factor. Over $S \times \mathbb{P} Q$ we get the short exact sequence

$$
0 \rightarrow \mathcal{F} \rightarrow \pi_{1}^{*} G \stackrel{j}{\rightarrow} \iota_{*}\left(q_{\mathbb{P} Q}\right) \rightarrow 0,
$$

where $j$ is the natural homomorphism and $\mathcal{F}$ its kernel. Given a point $\ell \in \mathbb{P} Q$, we denote by $\tilde{\ell} \subset G_{P}$ the corresponding hyperplane. The sheaf $\mathcal{F}_{\ell}, \ell \in \mathbb{P} Q$, is the subsheaf of $G$, with local sections whose values at $P$ belong to $\tilde{\ell}$. $\mathcal{F}_{\ell}$ is $H$ slope-stable, since $G$ is. $\mathcal{F}$ is thus a family of $H$-stable sheaves, flat over $\mathbb{P} Q$. Let $\kappa: \mathbb{P} Q \rightarrow M_{H}(v)$ be the classifying morphism associated to $\mathcal{F}$. The morphism $\kappa$ is clearly injective. An elementary calculation verifies that the differential $d_{\ell} \kappa$ is injective.

Let us compose the Mukai isomorphism $\theta$, given in (2.2), with pullback by $\kappa$ :

$$
v^{\perp} \stackrel{\theta}{\longrightarrow} H^{2}\left(M_{H}(v), \mathbb{Z}\right) \stackrel{\kappa^{*}}{\longrightarrow} H^{2}(\mathbb{P} Q, \mathbb{Z}) .
$$

The composition is given by

$$
\kappa^{*}(\theta(x))=c_{1}\left\{\pi_{2 !}\left[\pi_{1}^{!}\left(x^{\vee}\right) \otimes \mathcal{F}\right]\right\} .
$$


Let $[\mathcal{F}]$ be the class of $\mathcal{F}$ in $K(S \times \mathbb{P} Q)$. Then $[\mathcal{F}]=\pi_{1}^{!}[G]-\iota_{!}\left[q_{\mathbb{P} Q}\right]$. We have the equalities

$$
\begin{aligned}
c_{1}\left\{\pi_{2 !}\left(\pi_{1}^{!}\left(x^{\vee} \otimes G\right)\right)\right\} & =0, \\
c_{1}\left\{\pi_{2 !}\left(\pi_{1}^{!}\left(x^{\vee}\right) \otimes \iota_{!}\left(q_{\mathbb{P} Q}\right)\right)\right\} & =c_{1}\left\{\pi_{2 !}\left(\operatorname{rank}(x) \cdot \iota_{!}\left(q_{\mathbb{P} Q}\right)\right)\right\} \\
& =\operatorname{rank}(x) c_{1}\left(q_{\mathbb{P} Q}\right) .
\end{aligned}
$$

We conclude that the following equality holds, for all $x \in v^{\perp}$ :

$$
\int_{\mathbb{P} Q} \kappa^{*}(\theta(x))=-\operatorname{rank}(x) .
$$

A direct calculation verifies that the class $w$, given in (10.1), is orthogonal to $v$ and satisfies $(w, x)=-\operatorname{rank}(x)$, for all $x \in v^{\perp}$.

\section{EXAMPLE 10.15}

Let $S$ be a $K 3$ surface with a cyclic Picard group generated by an ample line bundle $H$. Let $b$ be an odd integer, such that there exists a line bundle $\mathcal{L} \in \operatorname{Pic}(S)$ of degree $2 n-4 b-2$, where $n>2$. If $c_{1}(\mathcal{L})$ is divisible by 2 , assume that $n>3$. Let $v \in K(S)$ be the class $(2, \mathcal{L},-b)$ in Mukai's notation. Then $(v, v)=2 n-2$ and the moduli space $M:=M_{H}(v)$ is smooth, projective, and $2 n$-dimensional.

Let $E \subset M$ be the closure of the locus of points representing $H$-stable sheaves $F$, which are not locally free, but such that $F^{* *} / F$ has length one. Let $Y$ be the normalization of the Uhlenbeck-Yau compactification of the moduli space of locally free $H$-slope stable sheaves of class $v$. Then $Y$ is a projective variety, and there exists a morphism $\phi: M \rightarrow Y$ whose exceptional locus contains $E$ (see [Li]).

\section{LEMMA 10.16}

$E$ is a prime exceptional divisor of type $A_{1}$. The class $[E] \in H^{2}(M, \mathbb{Z})$ is the primitive class $e:=\theta(2, \mathcal{L}, n-b-1)$. In particular, $(e, e)=2-2 n$.

(1) If the class $c_{1}(\mathcal{L})$ is not divisible by 2 , then $\operatorname{div}(e, \bullet)=n-1$,

$$
r s(e)= \begin{cases}\{1, n-1\} & \text { if } n \text { is even, } \\ \{1,(n-1) / 2\} & \text { if } n \text { is odd. }\end{cases}
$$

(2) If the class $c_{1}(\mathcal{L})$ is divisible by 2 , then $n \equiv 3$ (modulo 4$), \operatorname{div}(e, \bullet)=$ $2 n-2, r s(e)=\{2,(n-1) / 2\}$.

Proof

When the class $c_{1}(\mathcal{L})$ is divisible by 2 , then $n \equiv 3$ (modulo 4 ), since $\operatorname{deg}(\mathcal{L})=$ $2 n-4 b-2$ is divisible by 8 . In that case $n \geq 7$, by assumption.

If $F^{* *} / F$ has length one and $F$ is $H$-stable, then the reflexive hull $F^{* *}$ is necessarily $H$-semistable ${ }^{\dagger}$ of class $u:=(2, \mathcal{L}, 1-b) \in K(S) . M_{H}(u)$ is irreducible

${ }^{\dagger}$ The $H$-semistability is proven by an easy modification of the proof of [Ma4, Proposition 4.10, Part 1]. The assumption that the rank is 2 is needed. 
of dimension $2 n-4$, and its generic point represents a locally free $H$-slope-stable sheaf. This is clear if $c_{1}(\mathcal{L})$ is not divisible by 2 . If $c_{1}(\mathcal{L})$ is divisible by 2 , this follows from Lemma 10.9 and the assumption that $n \geq 7$.

Let $U \subset M_{H}(u)$ be the locus of $H$-stable locally free sheaves. Choose a twisted universal sheaf $\mathcal{G}$ over $S \times U$. Then $E$ contains a Zariski dense open subset isomorphic to the projectivization of $\mathcal{G}$. $E$ is irreducible, since the moduli space $M_{H}(u)$ is irreducible. We conclude that $E$ is a prime exceptional divisor, since it is contracted by the morphism to the Uhlenbeck-Yau compactification (also by Theorem 10.1). Furthermore, $E$ is of type $A_{1}$.

We calculate next the class $[E]^{\vee} \in H_{2}(M, \mathbb{Z})$, given in equation (3.2). Recall that $[E]^{\vee}$ is the class of the fiber of $E \rightarrow Y$, by Corollary 3.6. Fix an $H$-slopestable locally free sheaf $G$ of class $u \in K(S)$. Fix a point $P \in S$, and denote by $G_{P}$ the fiber of $G$ at $P$. Let $\mathbb{P} G_{P}$ be the projectivization of the fiber, and denote by

$$
\kappa: \mathbb{P} G_{P} \longrightarrow M_{H}(v)
$$

the morphism given in Lemma 10.14. Then $\kappa\left(\mathbb{P} G_{P}\right)$ is a fiber of $E \rightarrow Y$, and we get the equality $\left[\kappa\left(\mathbb{P} G_{P}\right)\right]=[E]^{\vee}$ of classes in $H_{2}(M, \mathbb{Z})$. We conclude that the following equalities hold, for all $x \in v^{\perp}$ :

$$
\int_{[E]^{\vee}} \theta(x)=-\operatorname{rank}(x)=\frac{1}{n-1}(w, x),
$$

where $w=(2, \mathcal{L}, n-b-1)$, by Lemma 10.14 .

The class $[E]$ is the unique class in $H^{2}(M, \mathbb{Z})$ satisfying the equality

$$
\frac{-2([E], \theta(x))}{([E],[E])}=\int_{[E]^{\vee}} \theta(x),
$$

for all $x \in v^{\perp}$, by Corollary 3.6. Now $(w, w)=2-2 n$, and so the equality $[E]=$ $\theta(w)$ follows from equation (10.2). Hence, $([E],[E])=(\theta(w), \theta(w))=2-2 n$. The class $[E]$ is primitive, since $w$ is.

Let us calculate $\operatorname{div}(e, \bullet)$. The class $x=\left(\rho, \mathcal{L}^{\prime}, \sigma\right)$ belongs to $v^{\perp}$ if and only if $b \rho=2 \sigma-\left(\mathcal{L}, \mathcal{L}^{\prime}\right)$. Hence, $(e, \theta(x))=(w, x)=(1-n) \rho$. If $c_{1}(\mathcal{L})$ is divisible by 2 , then every integral class $x \in v^{\perp}$ has even $\operatorname{rank} \rho$, and so $\operatorname{div}(e, \bullet)=2 n-2$. If $c_{1}(\mathcal{L})$ is not divisible by 2 , choose a line bundle $\mathcal{L}^{\prime}$, such that $\left(\mathcal{L}, \mathcal{L}^{\prime}\right)$ is odd, and set $\sigma:=\left[\left(\mathcal{L}, \mathcal{L}^{\prime}\right)+b\right] / 2$. Then $\left(1, \mathcal{L}^{\prime}, \sigma\right)$ belongs to $v^{\perp}$. Hence, $\operatorname{div}(e, \bullet)=n-1$.

The pair $(\widetilde{L}, e)$, given in equation $(6.1)$, may be chosen to consist of the saturation $\widetilde{L}$ in $K(S)$ of the lattice spanned by the classes $v$ and $w=\theta^{-1}(e)$, by Theorem 1.6 part 2.1. The largest integer dividing $w-v=(0,0, n-1)$ is $\sigma:=n-1$. Now $w+v=(4,2 \mathcal{L}, n-1-2 b)$. The largest integer $\rho$ dividing $w+v$ is 4 , if $c_{1}(\mathcal{L})$ is divisible by 2 . Otherwise, $\rho=1$, if $n$ is even, and $\rho=2$, if $n$ is odd. The invariant $r s(e)$ is then calculated via Table 1. 


\section{Examples of noneffective monodromy-reflective classes}

We provide examples of monodromy-reflective classes, which are not $\mathbb{Q}$-effective. Observation 1.12 guides us to lift these reflections to birational self-maps. Let us first prove the observation.

\section{Proof of Observation 1.12}

There exists a Zariski open subset $U \subset X$, such that $X \backslash U$ has codimension $\geq 2, \iota$ restricts to a regular involution of $U$, and the composition $H^{2}(X, \mathbb{Z}) \cong$ $H^{2}(U, \mathbb{Z}) \stackrel{\iota^{*}}{\longrightarrow} H^{2}(U, \mathbb{Z}) \cong H^{2}(X, \mathbb{Z})$ is an isometry, by [OG1, Proposition 1.6.2]. The isometry $\iota^{*}$ is assumed to be the reflection $R_{e}$. Hence, $\iota^{*} L \cong L^{-1}$ and $L$ is not $\mathbb{Q}$-effective.

Let $S$ be a projective $K 3$ surface with a cyclic Picard group generated by an ample line bundle $H$. Set $h:=c_{1}(H) \in H^{2}(S, \mathbb{Z})$. Set $d:=\operatorname{deg}(H) / 2$.

\subsection{Noneffective classes of divisibility $\operatorname{div}(e, \bullet)=2 n-2$}

Let $r, s$ be integers satisfying $s>r>2$ and $\operatorname{gcd}(r, s)=1$. Set $n:=r s+1$. Set $v:=(r, 0,-s), e:=\theta(r, 0, s)$, and $M:=M_{H}(v) . M$ is smooth and projective of dimension $2 n$. Let $\mathcal{L} \in \operatorname{Pic}(M)$ be the line bundle with class $e$. Let $\operatorname{Exc} \subset M$ be the locus of $H$-stable sheaves of class $v$, which are not locally free or not $H$ slope-stable. Exc is a closed subset of codimension $\geq 2$ in $M$, by Lemma 9.5. Let $M^{0}$ be the complement $M \backslash$ Exc of Exc, and let $\eta: M^{0} \rightarrow M$ be the inclusion. The restriction homomorphism $\eta^{*}: H^{2}(M, \mathbb{Z}) \rightarrow H^{2}\left(M^{0}, \mathbb{Z}\right)$ is an isomorphism. Let $\phi: M^{0} \rightarrow M^{0}$ be the involution sending a point representing the sheaf $F$, to the point representing $F^{*}$. Set $\psi:=\left(\eta^{*}\right)^{-1} \circ \phi^{*} \circ \eta^{*}$.

\section{PROPOSITION 11.1}

(1) The class $e$ is monodromy reflective, $(e, e)=2-2 n, \operatorname{div}(e, \bullet)=2 n-2$, and $r s(e)=\{r, s\}$.

(2) Let $R_{e}: H^{2}(M, \mathbb{Z}) \rightarrow H^{2}(M, \mathbb{Z})$ be the reflection by e. Then $R_{e}(\theta(\lambda))=$ $-\theta\left(\lambda^{\vee}\right)$, for all $\lambda \in v^{\perp}$.

(3) We have $\psi=R_{e}$.

(4) $H^{0}\left(M, \mathcal{L}^{k}\right)$ vanishes, for all nonzero integral powers $k$.

\section{Proof}

Part (1) was proven in Example 7.2. Set $\tilde{e}:=(r, 0, s) \in v^{\perp}$. Part (2) follows from the fact that $\theta: v^{\perp} \rightarrow H^{2}(M, \mathbb{Z})$ is an isometry, and the equality $R_{\tilde{e}}(\lambda)=-\lambda^{\vee}$, for all $\lambda \in v^{\perp}$. Part (4) follows from part (3), via Observation 1.12. We proceed to prove part (3). We need to prove the equality $\phi^{*}\left(\eta^{*}(y)\right)=\eta^{*} R_{e}(y)$, for all $y \in H^{2}(M, \mathbb{Z})$.

Let $\pi_{i}$ be the projection from $S \times M^{0}$ onto the $i$ th factor, $i=1,2$. Let $\mathcal{F}$ be a universal sheaf over $S \times M$, let $\mathcal{G}$ be its restriction to $S \times M^{0}$, and let [G] be 
its class in $K\left(S \times M^{0}\right)$. The morphism $\phi: M^{0} \rightarrow M^{0}$ satisfies

$$
(\mathrm{id} \times \phi)^{!} \mathcal{G} \cong\left(\mathcal{G} \otimes \pi_{2}^{*} A\right)^{*},
$$

for some line bundle $A \in \operatorname{Pic}\left(M^{0}\right)$. We have the commutative diagram

$$
\begin{array}{ccc}
K(S \times M) & \stackrel{(\mathrm{id} \times \eta)}{\longrightarrow} & K\left(S \times M^{0}\right) \\
\pi_{2 !} \downarrow & & \downarrow \pi_{2 !} \\
K(M) & \stackrel{\eta^{!}}{\longrightarrow} & K\left(M^{0}\right)
\end{array}
$$

by the Künneth theorem (see [At]). Hence,

$$
\eta^{*} \theta(x)=c_{1}\left[\pi_{2 !}\left(\pi_{1}^{!}\left(x^{\vee}\right) \otimes[\mathcal{G}]\right)\right],
$$

for all $x \in v^{\perp} \subset K(S)$. This explains the first equality below:

$$
\begin{aligned}
\phi^{*}\left(\eta^{*} \theta(x)\right) & =c_{1}\left\{\phi^{!} \pi_{2 !}\left(\pi_{1}^{!}\left(x^{\vee}\right) \otimes[\mathcal{G}]\right)\right\} \\
& =c_{1}\left\{\pi_{2 !}\left(\pi_{1}^{!}\left(x^{\vee}\right) \otimes(\mathrm{id} \times \phi)^{!}[\mathcal{G}]\right)\right\} \\
& =c_{1}\left\{\pi_{2 !}\left(\pi_{1}^{!}\left(x^{\vee}\right) \otimes\left([\mathcal{G}] \otimes \pi_{2}^{!}[A]\right)^{\vee}\right)\right\} \\
& =-c_{1}\left\{\pi_{2 !}\left(\pi_{1}^{!}(x) \otimes\left([\mathcal{G}] \otimes \pi_{2}^{!}[A]\right)\right)\right\} \\
& =\eta^{*} \theta\left(-x^{\vee}\right)=\eta^{*}\left(R_{e}(\theta(x))\right) .
\end{aligned}
$$

The fourth equality follows from Grothendieck-Verdier duality, the fifth is due to the fact that $\theta$ is independent of the choice of a universal sheaf, and the sixth follows from part (2).

\subsection{Noneffective classes of divisibility $\operatorname{div}(e, \bullet)=n-1$}

Let $r$ be a positive odd integer, let $\sigma$ be a positive integer, and set $n:=r \sigma+1$. Assume that $r \geq 3, \sigma \geq 3$, and $\operatorname{gcd}(r, \sigma)=1$. Let $S$ be a $K 3$ surface with a cyclic Picard group generated by an ample line bundle $H$. Set $d:=\operatorname{deg}(H) / 2$. Choose $(S, H)$, so that $\sigma$ and $d$ have the same parity. If $d$ is odd, assume that $\sigma>r$, possibly after interchanging $r$ and $\sigma$. Set $h:=c_{1}(H)$ and

$$
v:=(2 r, r h,-b),
$$

where $b:=[\sigma-r d] / 2$. Note that $\operatorname{gcd}(r, b)=\operatorname{gcd}(r, \sigma)=1$. Hence, $v$ is a primitive class in $K(S),(v, v)=2 n-2$, and the moduli space $M_{H}(v)$ is smooth and projective of type $K 3^{[n]}$. Let Exc $\subset M_{H}(v)$ be the locus parameterizing sheaves $F$, which are not locally free or not $H$-slope-stable. Exc is an algebraic subset of codimension $\geq 2$ in $M_{H}(v)$, by Lemma 9.6 .

Let $M^{0}$ be the complement $M \backslash$ Exc, and let $\eta: M^{0} \rightarrow M$ be the inclusion. Let $\phi: M^{0} \rightarrow M^{0}$ be the involution sending a point $[F]$, representing the sheaf $F$, to the point representing $F^{*} \otimes H$. The homomorphism $\eta^{*}: H^{2}(M, \mathbb{Z}) \rightarrow H^{2}\left(M^{0}, \mathbb{Z}\right)$ is an isomorphism, by Lemma 9.6. Set $\psi:=\left(\eta^{*}\right)^{-1} \circ \phi^{*} \circ \eta^{*}$.

Set $e:=(2 r, r h, \sigma-b)$. Note that $\operatorname{gcd}(r, \sigma-b)=\operatorname{gcd}(r, 2 \sigma-2 b)=\operatorname{gcd}(r, \sigma+$ $r d)=\operatorname{gcd}(r, \sigma)=1$. Hence, $e$ is a primitive class in $v^{\perp}$ of degree $(e, e)=2-2 n$. 


\section{LEMMA 11.2}

(1) The class $\theta(e)$ is monodromy reflective, and $\operatorname{div}(\theta(e), \bullet)=n-1$.

(2) We have $\widetilde{L}$ and $r s(e)= \begin{cases}H_{e v} \text { and }\{r, \sigma\} & \text { if } \sigma \text { is odd ( } n \text { even), } \\ U(2) \text { and }\{r, \sigma / 2\} & \text { if } \sigma \text { is even }(n \text { odd }) .\end{cases}$

(3) Let $R_{e}: v^{\perp} \rightarrow v^{\perp}$ be the reflection by e. Then $R_{e}(\lambda)=-\left[\lambda^{\vee}\right] \otimes H$, for all $\lambda \in v^{\perp}$.

(4) We have $\psi=R_{e}$.

(5) Let $\mathcal{L}$ be the line bundle on $M_{H}(v)$ with $c_{1}(\mathcal{L})=\theta(e)$. Then the vector space $H^{0}\left(M_{H}(v), \mathcal{L}^{k}\right)$ vanishes, for all nonzero integral powers $k$.

\section{Proof}

(1) Let $\lambda:=(x, c, y) \in K(S)$. Then $\lambda$ belongs to $v^{\perp}$ if and only if $r(h, c)-2 r y+$ $b x=0$. In particular, $x$ is divisible by $r$, since $\operatorname{gcd}(r, b)=1$. Now $(\lambda, e)=(\lambda, v)-$ $x \sigma=-x \sigma$. Thus $(e, \lambda)$ is divisible by $r \sigma=n-1$. There exists a class $c \in H^{2}(S, \mathbb{Z})$, satisfying $(c, h)=-b$, since the class $h$ is primitive and $H^{2}(S, \mathbb{Z})$ is unimodular. Then the class $\lambda:=(r, c, 0)$ belongs to $v^{\perp}$, and $(e, \lambda)=-r \sigma=1-n$. Hence, $\operatorname{div}(\theta(e), \bullet)=n-1$. The class $\theta(e)$ is monodromy reflective, by Proposition 1.5.

(2) If $\sigma$ is odd, then $n=r \sigma+1$ is even, and $\widetilde{L} \cong H_{e v}$, by Lemma 7.3. Now $(e-v) / \sigma=(0,0,1)$ is primitive. Hence, $r s(e)=\{r, \sigma\}$, by Lemma 6.5. If $\sigma$ is even, then $n$ is odd and $d$ is even. The classes $\alpha:=(e-v) / \sigma=(0,0,1)$ and $\beta:=(e+v) / 2 r=(2, h, d / 2)$ are integral isotropic classes, and $(\alpha, \beta)=-2$. Hence, $\{\alpha, \beta\}$ spans the primitive sublattice $\widetilde{L} \cong U(2)$. Consequently, $r s(e)=\{r, \sigma / 2\}$, by Lemma 6.5 .

Part (3) is verified by a straightforward calculation. Part (4) follows from part (3) by the same argument used in the proof of Proposition 11.1. Part (5) follows from part (4), by Observation 1.12.

\section{EXAMPLE 11.3}

Let $r$ and $s$ be positive integers satisfying $s>r$, one of $r$ or $s$ be even, and $\operatorname{gcd}(r, s)=1$. Set $n=4 r s+1$. Note that $n \equiv 1$ (modulo 8 ). Let $S$ be a $K 3$ surface with a cyclic Picard group generated by an ample line bundle $H$. Set $h:=c_{1}(H)$ and $d:=(h, h) / 2$. Assume that $d$ is odd. Then $s+r d$ is odd, since $r$ and $s$ consist of one odd and one even integer, by assumption. Set $v:=(4 r, 2 r h,-s+r d)$ and $e:=(4 r, 2 r h, s+r d)$. Then the classes $v$ and $e$ are primitive, $(e, v)=0$, $(v, v)=2 n-2$, and $(e, e)=2-2 n$. We have $(e+v) / 2 r=(4,2 h, d)$ and $(e-$ $v) / 2 s=(0,0,1)$. We claim that $\operatorname{div}(e, \bullet)=n-1$. Indeed, if $\lambda=(x, c, y)$ belongs to $v^{\perp}$, then $2 r$ divides $x$. Hence, $(e, \lambda)=(v, \lambda)-2 s x=-2 s x$ is divisible by $4 r s=n-1$. Furthermore, let $c$ be a class in $H^{2}(S, \mathbb{Z})$ satisfying $(c, h)=r d-s$, and set $\lambda=(2 r, c, 0)$. Then $(\lambda, e)=1-n$. Hence, $\operatorname{div}(e, \bullet)=n-1$. Hence, $\widetilde{L}=H_{e v}$, by Proposition 6.3. Thus $r s(e)=\{r, s\}$, by Lemma 6.5. Let Exc $\subset M_{H}(v)$ be the locus parameterizing sheaves which are not locally free or not $H$-slope-stable. Exc is an algebraic subset of codimension $\geq 2$ in $M_{H}(v)$, by the proof of the odd $d$ case of Lemma 9.6. (The symbol $r$ in that proof should remain $\operatorname{rank}(v) / 2$ and so should be set equal to twice the symbol $r$ above, and similarly the symbol $s$ in that 
proof should be set equal to twice the symbol $s$ above.) Let $\mathcal{L}$ be the line bundle over $M_{H}(v)$ with $c_{1}(\mathcal{L})=\theta(e)$. We conclude that $H^{0}\left(M_{H}(v), \mathcal{L}^{k}\right)$ vanishes, for all nonzero integers $k$, by the same argument used to prove Lemma 11.2.

Acknowledgments. This work was influenced by S. Druel's talk at the workshop "Holomorphically symplectic varieties and moduli spaces," which took place at the Université des Sciences et Technologies de Lille, in June 2009. I thank S. Druel for his clear exposition of his interesting work [D] on the divisorial Zariski decomposition and for correcting inaccuracies in an earlier draft of this paper. I would like to express my gratitude to the organizers, Dimitri Markushevich and Valery Gritsenko, for the invitation to participate in the workshop, for their hospitality, and for their wonderful work organizing this stimulating workshop. The paper is influenced by the work of Brendan Hassett and Yuri Tschinkel [HT1], [HT2]. I thank them for communicating to me a draft of their recent preprint [HT3] on the subject. Section 5 elaborates on ideas found already in [HT3]. I thank Brendan also for reading an early draft of the proof of Theorem 1.2. I thank Misha Verbitsky for answering my questions about his preprint [Ver]. I thank Valery Gritsenko for pointing out reference [GHS, Corollary 3.4]. I thank Kota Yoshioka for pointing out the relationship between Sections 2 and 3 of his paper [Y3], and Section 9.2 above.

\section{References}

[At] M. F. Atiyah, K-Theory, lecture notes by D. W. Anderson, W. A. Benjamin, New York, 1967. MR 0224083.

[BHPV] W. Barth, K. Hulek, C. Peters, and A. Van de Ven, Compact Complex Surfaces, 2nd ed., Ergeb. Math. Grenzgeb. (3) 4, Springer, Berlin, 2004. MR 2030225.

[Be1] A. Beauville, Varietes Kähleriennes dont la premiere classe de Chern est nulle, J. Differential Geom. 18 (1983), 755-782. MR 0730926.

[Be2] _ Symplectic singularities, Invent. Math. 139 (2000), 541-549. MR 1738060. DOI 10.1007/s002229900043.

[BCHM] C. Birkar, P. Cascini, C. Hacon, and J. McKernan, Existence of minimal models for varieties of log general type, J. Amer. Math. Soc. 23 (2010), 405-468. MR 2601039. DOI 10.1090/S0894-0347-09-00649-3.

[Bou] S. Boucksom, Divisorial Zariski decompositions on compact complex manifolds, Ann. Sci. École Norm. Sup. (4) 37 (2004), 45-76. MR 2050205. DOI 10.1016/j.ansens.2003.04.002.

[D] S. Druel, Quelques remarques sur la decomposition de Zariski divisorielle sur les varietes dont la premire classe de Chern est nulle, Math. Z. 267 (2011), 413-423. MR 2772258. DOI 10.1007/s00209-009-0626-4. 
[GHS] V. A. Gritsenko, K. Hulek, and G. K. Sankaran, The Kodaira dimension of the moduli of K3 surfaces, Invent. Math. 169 (2007), 519-567.

MR 2336040. DOI 10.1007/s00222-007-0054-1.

[HT1] B. Hassett and Y. Tschinkel, Rational curves on holomorphic symplectic fourfolds, Geom. Funct. Anal. 11 (2001), 1201-1228. MR 1878319. DOI 10.1007/s00039-001-8229-1.

[HT2] Moving and ample cones of holomorphic symplectic fourfolds, Geom. Funct. Anal. 19 (2009), 1065-1080. MR 2570315.

DOI 10.1007/s00039-009-0022-6.

[HT3] Monodromy and rational curves on holomorphic symplectic fourfolds, preprint, 2009.

[Hu1] D. Huybrechts, Compact hyperkähler manifolds: Basic results, Invent. Math. 135 (1999), 63-113; Erratum, Invent. Math. 152 (2003), 209-212.

[Hu2] _ "Compact hyperkähler manifolds" in Calabi-Yau manifolds and related geometries (Nordfjordeid, Norway, 2001), Universitext, Springer, Berlin, 2003, 161-225. MR 1963562.

[Hu3] , The Kähler cone of a compact hyperkähler manifold, Math. Ann. 326 (2003), 499-513. MR 1992275.

[Hu4] A global Torelli theorem for hyperkähler manifolds (after Verbitsky), Astérisque 348 (2012), Séminaire Bourbaki 2010-2011, no. 1040.

[HL] D. Huybrechts, and M. Lehn, The Geometry of Moduli Spaces of Sheaves, Aspects Math. E31, Vieweg, Braunschweig, 1997. MR 1450870.

[KLS] D. Kaledin, M. Lehn, and C. Sorger, Singular symplectic moduli spaces, Invent. Math. 164 (2006), 591-614. MR 2221132.

DOI 10.1007/s00222-005-0484-6.

[Ka] Y. Kawamata, Unobstructed deformations, II, J. Algebraic Geom. 4 (1995) 277-279; Erratum, J. Algebraic Geom. 6 (1997), 803-804. MR 1311350.

[KM] J. Kollár and S. Mori, Classification of three-dimensional flips, J. Amer. Math. Soc. 5 (1992), 533-703. MR 1149195. DOI 10.2307/2152704.

[Le] J. Le Potier, Systémes cohérents et structures de niveau, Astérisque 214, Soc. Math. France, Montrouge, 1993. MR 1244404.

[Li] J. Li, Algebraic geometric interpretation of Donaldson's polynomial invariants, J. Differential Geom. 37 (1993), 417-466. MR 1205451.

[Ma1] E. Markman, Brill-Noether duality for moduli spaces of sheaves on K3 surfaces, J. Algebraic Geom. 10 (2001), 623-694. MR 1838974.

[Ma2] Integral generators for the cohomology ring of moduli spaces of sheaves over Poisson surfaces, Adv. Math. 208 (2007), 622-646. MR 2304330. DOI 10.1016/j.aim.2006.03.006.

[Ma3] On the monodromy of moduli spaces of sheaves on K3 surfaces, J. Algebraic Geom. 17 (2008), 29-99. MR 2357680.

DOI 10.1090/S1056-3911-07-00457-2. 
[Ma4] Integral constraints on the monodromy group of the hyperkähler resolution of a symmetric product of a K3 surface, Internat. J. Math. 21 (2010), no. 2, 169-223. MR 2650367. DOI 10.1142/S0129167X10005957.

[Ma5] Modular Galois covers associated to symplectic resolutions of singularities, J. Reine Angew. Math. 644 (2010), 189-220. MR 2671779. DOI 10.1515/CRELLE.2010.056.

[Ma6] _ _ _A survey of Torelli and monodromy results for hyperkahler manifolds" in Complex and Differential Geometry, Springer Proc. Math. 8, Springer, Heidelberg, 2011, 257-323. MR 2964480.

DOI 10.1007/978-3-642-20300-8_15.

[Ma7] On the monodromy of moduli spaces of sheaves on K3 surfaces, II, preprint, arXiv:math.AG/0305043v4 [math.AG]

[Mat] H. Matsumura, Commutative Algebra, W. A. Benjamin, New York, 1970. MR 0266911.

[Mu] S. Mukai, "On the moduli space of bundles on K3 surfaces, I" in Vector Bundles on Algebraic Varieties (Bombay, 1984), Tata Inst. Fund. Res. Stud. Math. 11, Oxford Univ. Press, 1987, 341-413. MR 0893604.

[Na1] Y. Namikawa, Deformation theory of singular symplectic $n$-folds, Math. Ann. 319 (2001), 597-623. MR 1819886. DOI 10.1007/PL00004451.

[Na2] Poisson deformations of affine symplectic varieties, II, Kyoto J. Math. 50 (2010), 727-752. MR 2740692. DOI 10.1215/0023608X-2010-012.

[Ni] V. V. Nikulin, Integral symmetric bilinear forms and some of their applications, Izv. Math. 14 (1980), 103-167.

[O] K. Oguiso, K3 surfaces via almost-primes, Math. Res. Lett. 9 (2002), 47-63. MR 1892313.

[OG1] K. O'Grady, The weight-two Hodge structure of moduli spaces of sheaves on a K3 surface, J. Algebraic Geom. 6 (1997), 599-644. MR 1487228.

[OG2] _ _ Desingularized moduli spaces of sheaves on a K3, J. Reine Angew. Math. 512 (1999), 49-117. MR 1703077. DOI 10.1515/crll.1999.056.

[R] Z. Ran, Hodge theory and the Hilbert scheme, J. Differential Geom. 37 (1993), 191-198. MR 1198605.

[Ver] M. Verbitsky, A global Torelli theorem for hyperkahler manifolds, preprint, arXiv:0908.4121v7 [math.AG]

[Voi] C. Voisin, Hodge Theory and Complex Algebraic Geometry, I, Cambridge Stud. Adv. Math. 76, Cambridge Univ. Press, Cambridge, 2002. MR 1967689. DOI 10.1017/CBO9780511615344.

[W] J. Wierzba, Contractions of symplectic varieties, J. Algebraic Geom. 12 (2003), 507-534. MR 1966025. DOI 10.1090/S1056-3911-03-00318-7.

[Y1] K. Yoshioka, Some examples of Mukai's reflections on K3 surfaces, J. Reine Angew. Math. 515 (1999), 97-123. MR 1717621.

DOI 10.1515/crll.1999.080. 
[Y2] Moduli spaces of stable sheaves on abelian surfaces, Math. Ann. 321 (2001), 817-884. MR 1872531. DOI 10.1007/s002080100255.

[Y3] I I I _ _ - Ireducibility of moduli spaces of vector bundles on K3 surfaces, preprint, arXiv:math/9907001v2 [math.AG]

Department of Mathematics and Statistics, University of Massachusetts, Amherst, Massachusetts 01003, USA; markman@math.umass.edu 